\title{
Review \\ E-Health Practices and Technologies: A Systematic Review from 2014 to 2019
}

\author{
Maria Helena da Fonseca ${ }^{1, *(\mathbb{C}}$, Fanny Kovaleski ${ }^{1}$, Claudia Tania Picinin ${ }^{1}\left(\mathbb{D}\right.$, Bruno Pedroso $^{2}(\mathbb{C}$ \\ and Priscila Rubbo ${ }^{3}$
}

1 Department of Production Engineering, Federal University of Technology—Paraná (UTFPR), Ponta Grossa 84017-220, Brazil; fannyk92@hotmail.com (F.K.); claudiapicinin@utfpr.edu.br (C.T.P.)

2 Division of Physical Education, State University of Ponta Grossa-Paraná (UEPG), Ponta Grossa 84030-900, Brazil; prof.brunopedroso@gmail.com

3 Department of Accounting Sciences, Federal University of Technology—Paraná (UTFPR), Pato Branco 85503-390, Brazil; priscilarubbo@utfpr.edu.br

* Correspondence: mhelfonseca13@gmail.com; Tel.: +55-42-999388129

check for updates

Citation: da Fonseca, M.H.;

Kovaleski, F.; Picinin, C.T.; Pedroso,

B.; Rubbo, P. E-Health Practices and Technologies: A Systematic Review from 2014 to 2019. Healthcare 2021, 9 , 1192. https://doi.org/10.3390/ healthcare 9091192

Academic Editor: Justin W. Collins

Received: 16 July 2021

Accepted: 26 August 2021

Published: 10 September 2021

Publisher's Note: MDPI stays neutral with regard to jurisdictional claims in published maps and institutional affiliations.

Copyright: (c) 2021 by the authors. Licensee MDPI, Basel, Switzerland. This article is an open access article distributed under the terms and conditions of the Creative Commons Attribution (CC BY) license (https:// creativecommons.org/licenses/by/ $4.0 /)$.

\begin{abstract}
E-health can be defined as a set of technologies applied with the help of the internet, in which healthcare services are provided to improve quality of life and facilitate healthcare delivery. As there is a lack of similar studies on the topic, this analysis uses a systematic literature review of articles published from 2014 to 2019 to identify the most common e-health practices used worldwide, as well as the main services provided, diseases treated, and the associated technologies that assist in e-health practices. Some of the key results were the identification of the four most common practices used (mhealth or mobile health; telehealth or telemedicine; technology; and others) and the most widely used technologies associated with e-health (IoT, cloud computing, Big Data, security, and systems).
\end{abstract}

Keywords: e-health; ehealth; practices; technologies

\section{Introduction}

Digital change has been particularly challenging in healthcare, as there is growing demand for services due to the aging population and the emergence of new diseases. Thus, investment in new treatments is necessary so that everyone has equal access to the healthcare system [1-3]. E-health involves practices such as mhealth and telehealth that employ electronic technologies to provide healthcare resources, services, and information [4].

Mobile health or mhealth consists of the use of mobile devices so that patients can solicit services electronically, use apps to verify information, and manage or monitor treatment or problems or other health-related issues [5]. Telehealth can be defined as the use of telecommunication technologies to promote the care and education of patients and professionals working in the area [6].

E-health has become an integral part of the healthcare system as it addresses a range of difficulties in medicine, including reducing errors and providing more efficient services with more accurate results [7]. Such is the case with the use of electronic medical records, in which all information about a patient is stored, thus preventing inappropriate administration of medication during medical care and ensuring that the patient is treated quickly and comfortably [8]. However, its implementation depends on adequate planning and strategies so that virtual medical care can be performed [3].

The success of e-health in a country is related to several factors, including user acceptance and the types of infrastructure, systems, and management used [6,9]. Meanwhile, there are four stakeholders involved in the outcomes: entrepreneurs, healthcare professionals, patients, and those responsible for health insurance and assistance policies [10]. To effectively implement the use of information technologies in healthcare, e-health strategies must occur in an integrated manner, including the development of norms, laws, or regulations. This situation is valid whether in the fields of telehealth and mhealth, or specific 
categories such as electronic medical records or health literacy-eLearning (learning in health) $[5,11,12]$.

The e-health strategy has three main components: (i) knowledge management; (ii) tools and methods; and (iii) policies. These components work to consolidate healthcare systems with support networks and scientific and technological production, manage infrastructure and human resources, reduce barriers to accessing health services, and promote community inclusion [13]. Some barriers to e-health include the difficulties faced when using systems and applications, both by healthcare professionals and patients, as well as ensuring the security and privacy of user data transmitted throughout these systems [12].

Another challenge in e-health is interoperability across systems. That is, new e-health systems must interact with existing ones, and there should be a standard electronic language between hospitals (or clinics) to facilitate communication and data exchange, as well as formal agreements on how the system should work in a standardized way $[14,15]$. Further, the cost of implementing e-health also presents challenges, which may make the implementation of such systems unfeasible. This is related to the high levels of investment required to purchase equipment to implement more sustainable practices $[3,16]$ than traditional systems that store paper records, alongside the costs of hiring specialized support personnel in information technology (IT) to keep the systems running and software acquisition $[8,17]$.

There are several studies on the topic of e-health, but they generally address a specific practice. One example is [18], in which the authors analyze studies of interventions via e-health using websites and social media in the treatment of patients with mood disorders. Two e-health-related studies with a general approach were found [19,20]. However, such studies do not fully address all e-health practices, which differentiates the present study from previous work on the topic. Although scientific publication databases have a vast range of studies on e-health [21], there are gaps in knowledge related to this topic, which justifies the need for the present review. Herein, we update data related to practices in the field of e-health and provide an overview of the information present in selected articles within a five-year time frame (2014 to 2019), demonstrating what has been published on the topic both in practical studies and literature reviews.

Therefore, the objective of this analysis is to identify, through a systematic literature review, the most commonly used e-health practices worldwide, as well as the key services provided, diseases treated, and the associated technologies that assist in providing e-health practices. This study aims to answer the following questions:

- In which countries and journals are studies published on e-health practices?

- What are the main e-health practices used worldwide?

- What are the main service delivery types and medical fields addressed using e-health?

- What are the main barriers to e-health service delivery?

- What are the most common diseases treated, and in which countries have e-health practices been applied?

- What are the most common technologies used in e-health?

\section{Materials and Methods}

The identification of the study portfolio was carried out using the Methodi Ordinatio process developed [22] and based on the Cochrane model and ProKnow-C for research studies that use Information and Communication Technologies-ICTs, such as spreadsheets, word processors, and reference managers. The following software programs were employed to conduct the analyses: Mendeley version 1.19.3; JabRef version 3.3; Microsoft Office 365 (Excel and Word); and NVivo trial version 11 for initial article analysis. The Methodi Ordinatio includes nine steps and is used to conduct systematic literature reviews, build bibliographic portfolios, and map the literature on a specific topic. One advantage of the methodology is its multi-criteria decision-making model that considers the impact factor of the journal, the number of citations, and the year of publication. From this, the 
InOrdinatio index is calculated, and the researcher obtains a bibliographic portfolio [23].

This methodology has been used by authors such as [24-26].

The Methodi Ordinatio is shown in Figure 1 and is composed of the following steps:

1. Step 1-Establish the research objective;

2. Step 2-Define the keywords;

3. Step 3-Select the databases to be searched;

4. Step 4-Search for and register the results obtained;

5. Step 5-Define search filters such as publication period and type of study (articles, books, etc.), excluding duplicate articles and those unrelated to the selected research topic;

6. Step 6-Identify the impact factor, year of publication, and number of citations of the articles from Google Scholar for the selected portfolio;

7. Step 7-Apply the InOrdinatio equation [22] in a spreadsheet to classify the identified articles;

InOrdinatio $=(I F \div 1000)+\alpha \times[10-($ ResearchYear - PublishYear $)]+\left(\sum C i\right)$

The equation is composed of the following variables: $I F$ is the impact factor; $\alpha$ is the value defined by the researchers considering the current relevance of the articles, which may vary from 1 to 10 (for this study, we defined $\alpha$ as 10); ResearchYear is the year the research was conducted; PublishYear is the year the article was published; and $\Sigma C i$ is the number of citations of the article according to data from Google Scholar.

8. Step 8-Select the articles for the final portfolio considering the highest InOrdinatio classification; and

9. Step 9-Read and analyze the final portfolio articles.

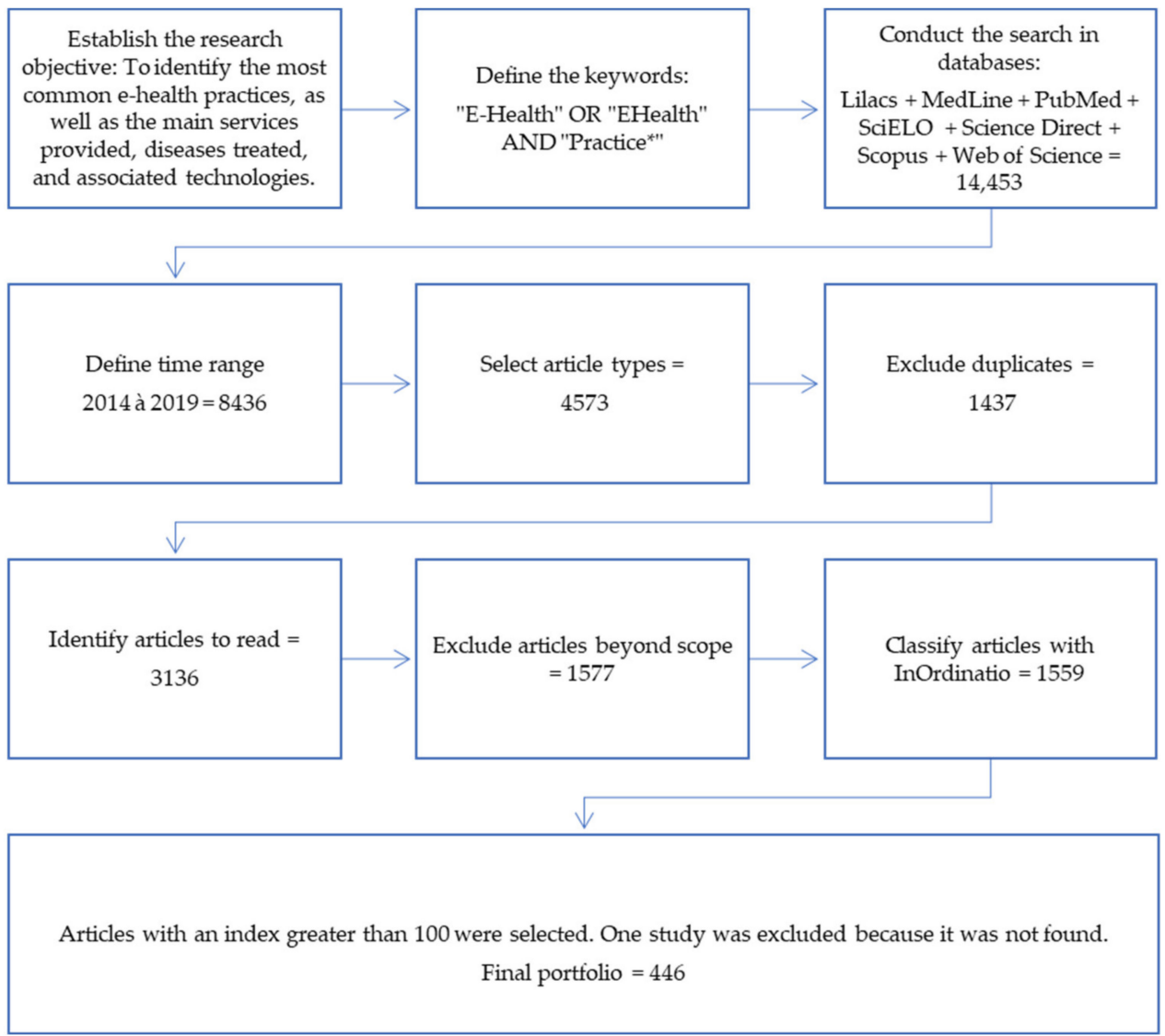

Figure 1. Research method. 
First, the search objective was defined, and the keywords were searched using Boolean operators ("E-Health" OR “EHealth" AND "Practice *"). The keyword "Technology" was not used to search the databases so as not to excessively limit the search. The analysis of e-health-related technologies took place after the portfolio was defined.

The search was carried out using seven electronic databases: Lilacs, MedLine, PubMed, SciELO, Scopus, Science Direct, and Web of Science. These databases were chosen due to the multidisciplinary character of this study and the fact that the information concerning medicine and health is vast. Thus, to conduct a careful analysis, well-known and key sources in the field should be used, such as MedLine, PubMed, Lilacs, SciELO, the Cochrane Library, and the Virtual Health Library (VHL).

MedLine is a database containing supplementary materials and offers a good basis for research as it indexes other databases and aggregates complementary materials. It has been employed in research on e-health, health, and medical technology [6].

Scopus is the largest research database for the fields of technology, medicine, science, social sciences, and the arts and humanities, while Science Direct contains technical publications in the fields of science and health [27]. The Web of Science is an independent global citation database that offers reliable multidisciplinary data for academic research [28].

The time frame for the published research was five years, from 2014 to 2019. According to [29], a five-year period is the half-life for article citations. In addition, the following filtering procedures were applied to the databases to obtain the results: selection of types of articles published (review, research, and journal-published), exclusion of duplicate articles. Thus, 3136 articles were obtained, whose titles, abstracts and keywords were read to exclude studies that dealt with areas unrelated to e-health practices and are beyond the scope of this analysis.

Finally, the Methodi Ordinatio equation was applied to obtain the most currently relevant articles. Articles with an index greater than 100 were considered, and one study was excluded as it was not found, resulting in a final portfolio of 446 articles, as listed in Appendix A-Table A1. Final portfolio.

The database search did not exclude any publication language, aiming to obtain a broad range of results and not limit the present analyses. Table 1 shows how the studies were analyzed to answer the research questions.

Table 1. Type of analysis used to address the research questions.

\begin{tabular}{cc}
\hline Type of Analysis & Research Question \\
\hline Bibliometric analysis & In which countries and journals are studies on e-health practices published? \\
Content analysis & What are the main e-health practices used worldwide? \\
What are the main barriers to e-health service delivery? \\
What are the $\begin{array}{c}\text { most commonly treated diseases, and in which countries has e-health } \\
\text { been applied? }\end{array}$ \\
What are the most common technologies in e-health?
\end{tabular}

The questions referring to countries that are publishing studies on e-health and countries that use these practices were answered, respectively, by identifying the country of the first author and where the studies took place. Thus, the results and discussions of the research were organized in a bibliometric and content analysis carried out on the 446 articles from the final portfolio. For the content analysis of the technologies associated with e-health, 57 articles from the final portfolio (446 articles) were analyzed as they specifically address this topic.

\section{Results and Discussion}

\subsection{Bibliometric Analysis}

Figure 2 shows the number of publications per year from 2014 to 2019. 


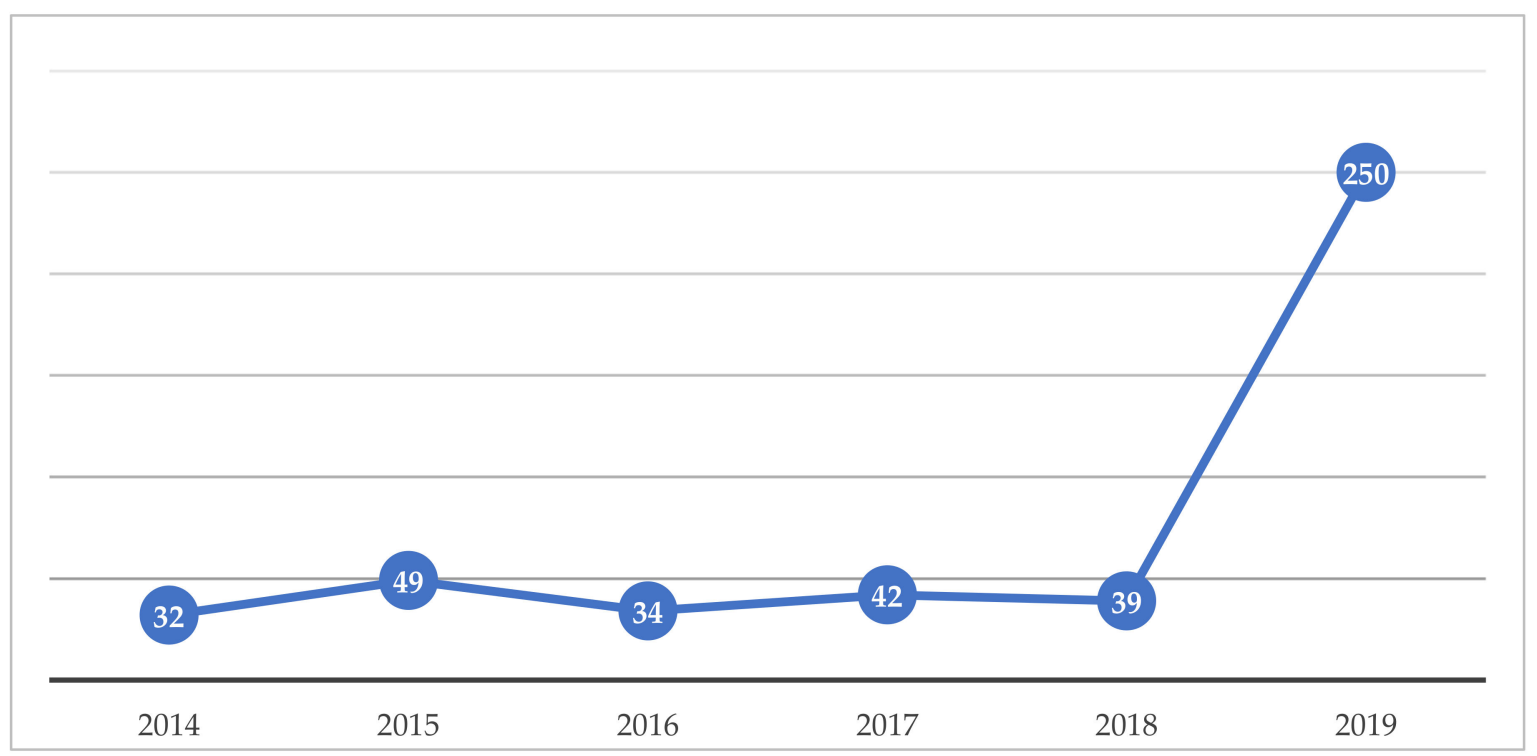

Figure 2. Number of publications per year.

We can see that 2014 had the lowest number of publications on the topic and 2019 the highest. There was fluctuation in the number of publications, particularly between 2018 and 2019, when a marked increase in the number of studies related to the theme of e-health occurred. This increase is related to several factors. The area of mhealth has been growing, as has the number of chronic diseases and other health problems, while the advance of mobile technology has enabled greater access to e-health through devices, enabling an increase in research [30]. An increase in publication on the topic is also due to researcher interest, as it is current and still novel, allowing for greater numbers of studies on topics within the field, as is the case with reports published about e-health indicators, such as those from the Global Health Observatory of the World Health Organization (WHO) and the Nordic e-Health Research Network [31].

Although authors from 58 countries contributed to studies, the geographical distribution was uneven, as shown in Figure 3.
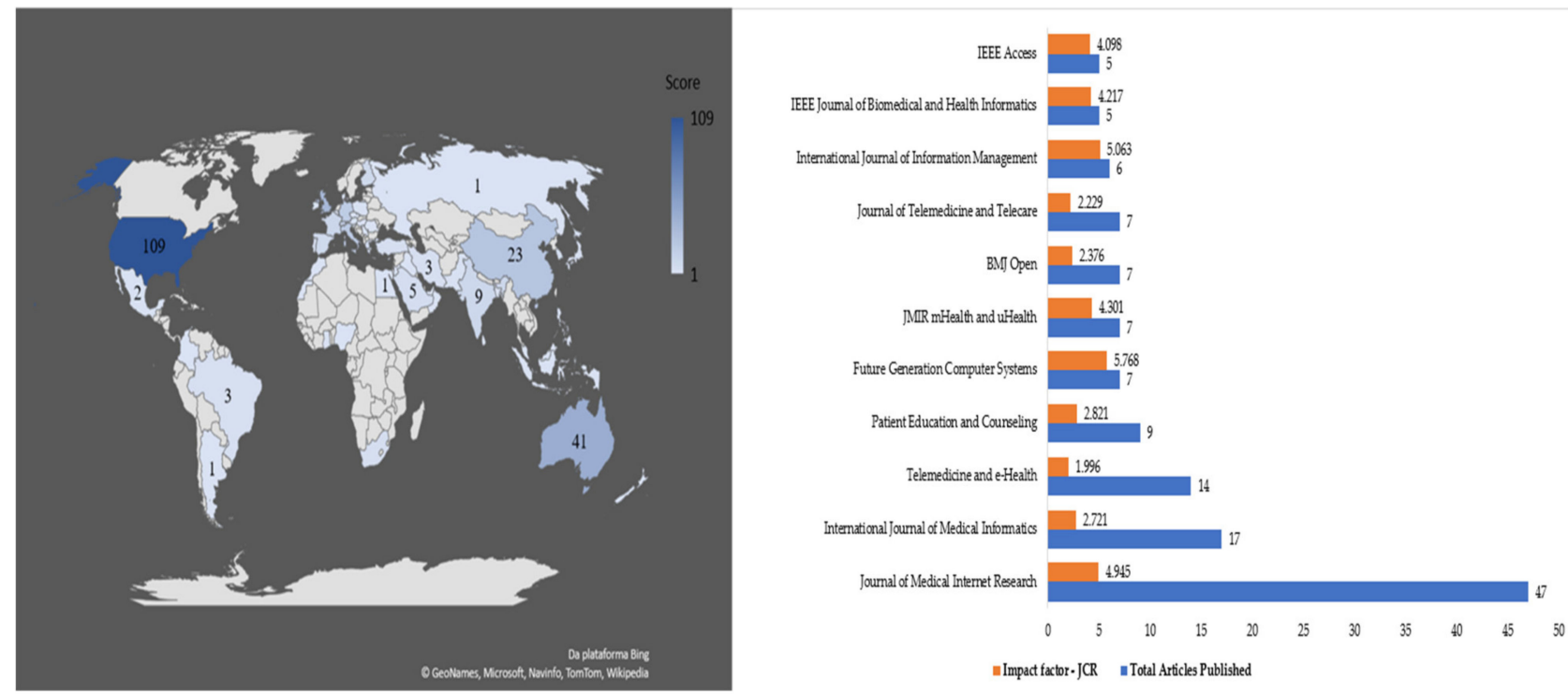

Figure 3. Distribution of articles by country and number of articles published by journal. 
The leading countries in developing research related to e-health are United States (109), Australia (41), United Kingdom (32), China (23), Italy (19), Germany (18), Norway (14), and France (10). The countries with the fewest publication include Egypt (1), Mexico (2), and Brazil (3). In the study by [32] on e-health and health informatics skills, the authors found that the countries with the most authors who published on the topic were United States, Canada, United Kingdom, and Australia, and those with the least were Denmark and Norway.

Norway, Australia, Germany, the United Kingdom, United States, France, and Italy are countries with a remarkably high Human Development Index-HDI (which is based on the health, life expectancy, education, and income of a country's population), while Egypt, Brazil, Mexico, and China are countries with high HDI. The wealthier a country is, the better its economic, political, and healthcare infrastructure, enabling more initiatives for e-health development, as has occurred in many European countries [33,34]. Each country has a different level of e-health development, and consequently, countries with more advanced e-health systems have a higher incidence of scientific production on the topic, such as European countries and the United States, as verified by [35]. Moreover, [36] state that although countries on the African continent and Latin America are publishing on the topic, the number of studies is still low as the countries have less developed e-health systems. Such is the case with Brazil and Mexico, which are still at the stage of telehealth improvement and consolidation.

The 446 articles in the portfolio were published across 253 academic journals. However, some journals among those identified stand out for having very few publications on the topic but a high impact factor, such as The Lancet (1 publication-60.392 JCR); British Medical Journal-BMJ (1 publication-30.223 JCR); IEEE Communications Surveys E Tutorials (1 publication-23.700 JCR); IEEE Internet of Things Journal (2 publications-9.936 JCR); Schizophrenia Bulletin (2 publications-7.958 JCR); Information Sciences (2 publications5.910 JCR); AIDS and Behavior (3 publications-3.147 JCR); Social Science E Medicine (3 publications-3.616 JCR); International Journal of Environmental Research and Public Health (3 publications-2.849 JCR); Journal of the American Medical Informatics Association-JAMIA (4 publications-4.112 JCR); Frontiers in Psychiatry (4 publications-2.849 JCR); PLoS One (4 publications-2.740 JCR).

In the analysis by [19], who conducted a literature review on e-health, and [32], who did a bibliometric study, the journals that had the most publications were Journal of Medical Internet Research and Telemedicine and e-Health.

Therefore, for the research question "In which country and journals are studies on e-health practices published worldwide?" we can see that the United States stands out as it has the greatest number of authors who published on this topic, with 109 studies. Moreover, the Journal of Medical Internet Research is the most prominent scientific outlet for research on e-health, with 47 publications.

\subsection{Content Analysis}

3.2.1. Main E-Health Service Delivery Types and Fields

Based on the survey of studies from the final portfolio, Table 2 presents the categories of practices and services provided in e-health as described in the analyzed studies.

Table 2. E-health practices and fields.

\begin{tabular}{ccc}
\hline E-Health Practices & $\begin{array}{c}\text { Specification of Service Delivery Types } \\
\text { and Other E-Health-Related Topics } \\
\text { Found in the Articles }\end{array}$ & Medical Fields Identified in the Articles \\
\hline Mhealth or Mobile Health & $\begin{array}{c}\text { Mhealth; assessment; systems assessment; } \\
\text { information; telehealth; monitoring; health } \\
\text { literacy. }\end{array}$ & $\begin{array}{c}\text { General medicine; emergency; pediatrics; cardiology; } \\
\text { oncology; psychiatry; neurology; dermatology; gynecology; } \\
\text { hematology; infectiology/infectious diseases; } \\
\text { radiotherapy/radiology; diagnostic imaging; } \\
\text { gastroenterology; anesthesiology; nutrition; orthopedics; } \\
\text { respiratory system/otorhinolaryngology; general surgery; } \\
\text { urology; geriatrics; endocrinology; nephrology; } \\
\text { ophthalmology }\end{array}$ \\
\hline
\end{tabular}


Table 2. Cont.

\begin{tabular}{|c|c|c|c|}
\hline E-Health Practices & $\begin{array}{l}\text { Specification of Service Delivery Types } \\
\text { and Other E-Health-Related Topics } \\
\text { Found in the Articles }\end{array}$ & Medical Fields Identified in the Articles & Authors \\
\hline Telehealth or Telemedicine & $\begin{array}{l}\text { Telehealth; intervention; interaction; } \\
\text { mhealth; systems; technology. }\end{array}$ & $\begin{array}{l}\text { Telepsychiatry; teledermatology; teledentistry; } \\
\text { telerehabilitation; teleophthalmology; telecardiology. }\end{array}$ & {$[43-48]$} \\
\hline Technology & $\begin{array}{l}\text { Technology; others; mhealth; patient } \\
\text { monitoring; support; systems; telehealth; } \\
\text { knowledge level; health literacy; systems } \\
\text { assessment; program. }\end{array}$ & & [49-54] \\
\hline Others & $\begin{array}{l}\text { Others; diagnosis; telehealth; mhealth; } \\
\text { costs; programs; problems; project; quality } \\
\text { of services; general e-health; care; project } \\
\text { assessment; benefits; development; } \\
\text { diagnosis; evaluation system; impact x } \\
\text { cost-benefit; use and acceptance/barriers; } \\
\text { knowledge level; mhealth; treatments; } \\
\text { professionals' views; study groups; } \\
\text { intervention assessment; health literacy; } \\
\text { technology; telehealth; randomized } \\
\text { controlled trial-RCT; } \\
\text { standardized service. }\end{array}$ & $\begin{array}{l}\text { Nursing; oncology; gynecology; psychology; neurology; } \\
\text { cardiology; psychiatry. }\end{array}$ & [55-66] \\
\hline
\end{tabular}

When answering the research question "What are the most common e-health practices used around the world?" four categories of e-health practices became evident: (i) mhealth or mobile health; (ii) telehealth or telemedicine; (iii) technology; and (iv) others, which include combinations of different practices.

E-health can be defined as healthcare services and health information provided and/or obtained using the internet, mobile devices, computers, and information technology [64]. It involves the application of digital solutions for healthcare, thus facilitating patient care in a more comfortable way [62]. The practice areas are described below.

Mhealth or mobile health enables persons who use mobile devices, such as smartphones, to access systems, data, and apps to monitor and manage their health status [38]. Telehealth or telemedicine includes remote consultations via videoconference/call using desktop computers, smartphones, or tablets with internet access $[44,67,68]$.

The technology applied in e-health helps healthcare professionals, patients, and the lay population to obtain information or access learning, treatment, and resources that are available online [69].

Through e-health, digital health interventions (DHIs), such as assisted therapy, have been shown as effective among children and young people undergoing mental health treatment, as discussed in the systematic review by [64] on randomized clinical trials of DHIs performed with children and young people up to 25 years of age. However, further data are required to offer satisfactory conclusions about the benefits; despite the potential that the intervention can offer, few studies have been carried out on the subject and with small sample sizes, making this assessment difficult.

In an analysis by [62], the authors evaluated the effectiveness of an electronic health project model for people with severe mental illness (SMI), called the Flat Explicit Design Model (FEDM). The study was carried out via a website with 38 people aged 31 to 59 years who have some degree of severe mental illness and involved online tests.

We also identified a study that sought to conduct a theoretical review on the services and applications offered in the practice of mhealth and their use for therapy in the areas of mental health and behavioral disorders, musculoskeletal and connective tissue systems, oncology, and the nervous system [38]. Another study assessed the skills of medical residents, doctors, and clinics working with telepsychiatry, with a focus on e-mental health (e-MH) care [44].

In the "other" category, articles describing a combination of past practices and other areas, such as costs, were included. The study by [56] assessed the current literature to verify whether specialization in telehealth offset the effective cost. The authors found specializations such as teledermatology, teleophthalmology, and telecardiology offset the amount invested in that healthcare service. 
Some of the service delivery types and other themes combined with the most representative e-health practices in the literature are discussed below.

Health literacy is knowledge and skills related to health, and it can be obtained through in-person and/or online environments [70]. The latter includes websites, social media, and the use of health data systems [71]. An aspect related to this area in the analyzed studies is low health literacy, as seen in the studies by [72,73]; when searching for health information, internet users must use a critical approach to determine if the information obtained is correct. Often, the information posted online is presented erroneously, which may misguide or misinform the user about the content.

Mhealth or mobile health and monitoring consists of using smartphone applications to help in disease treatment, identification, and support [74]. It also encompasses health exam scheduling and patient monitoring performed via devices with wireless sensors that check essential vital signs, such as blood glucose level and blood pressure [38]. The results of these exams are stored on the device, thus allowing for the transmission of data from exams done at home [75].

In telehealth, when an intervention is combined with interaction with a healthcare professional, it can be defined as telemonitoring of patients with diseases that need followup [43]. This enables doctors to see patients without having to go to the hospital or doctor's office [45]. Such a service delivery can be employed with mental health patients who require therapy [44], enabling them to participate in sessions at home at more flexible times [76]. It can also be used to monitor the symptoms of cancer patients undergoing treatment [77].

The systems related to the category "technology" entail the creation of encryptions to protect the data of patients who access their medical records online, as well as data protection in the systems of hospitals, pharmacies, and clinics $[52,53]$ so that data remain confidential and secure [54]. Technology also involves creating support for the systems [78] and developing mobile devices that can be used in the healthcare sector, such as wearable sensors [79]. Further, it includes the use of Internet of Things (IoT) [80], cloud storage [81], and Big Data [82] in the areas of e-health.

Regarding the research question "What are the main service delivery types and medical fields served in e-health?" we found that the main areas of service provision are health literacy, mhealth and monitoring, telehealth, and systems combined with technology. In turn, the main medical fields served that are highlighted in the literature are telepsychiatry, teledermatology, telerehabilitation, teleophthalmology, telecardiology, and teledentistry.

As for the research question "What are the main barriers to e-health service delivery?" we found that costs, laws, and system data security of are the main barriers to be overcome. We identified studies related to e-health practices experiencing barriers in terms of costs related to the use of telemedicine [83], regulation and legislation [38], security in the use of cloud computing [84], limitations in clinical trials of existing digital interventions [64], and limitations in databases [58]. In mHealth, a limiting factor is mobile device battery life [85], as the devices with applications or monitoring equipment must have the battery charged to avoid any problems during use [86]. Further, geographic distance is a primary limiting factor, as each country has different health-related applications available to the population [87] and barriers between patient and physician [38].

\subsubsection{Main Diseases Treated}

Figure 4 shows the main diseases treated in e-health (total and percentage), addressing the research question "What are the most commonly treated diseases and in which countries have e-health services been used?" The most commonly treated diseases are mental illnesses, multiple diseases (diabetes mellitus 1 and 2, stress, depression, and anxiety), cancer, eating disorders, chronic illness, cardiovascular diseases, and sexually transmitted diseases (STDs). Regarding the countries that currently use e-health, we identified the following countries where studies have been conducted: United States, Canada, Australia, Germany, Sub-Saharan Africa, Africa, Netherlands, Sweden, Switzerland, China, Italy, 
Greece, Finland, Iran, Iraq, Bangladesh, Pakistan, Saudi Arabia, United Kingdom, Spain, France, Italy, and Portugal.

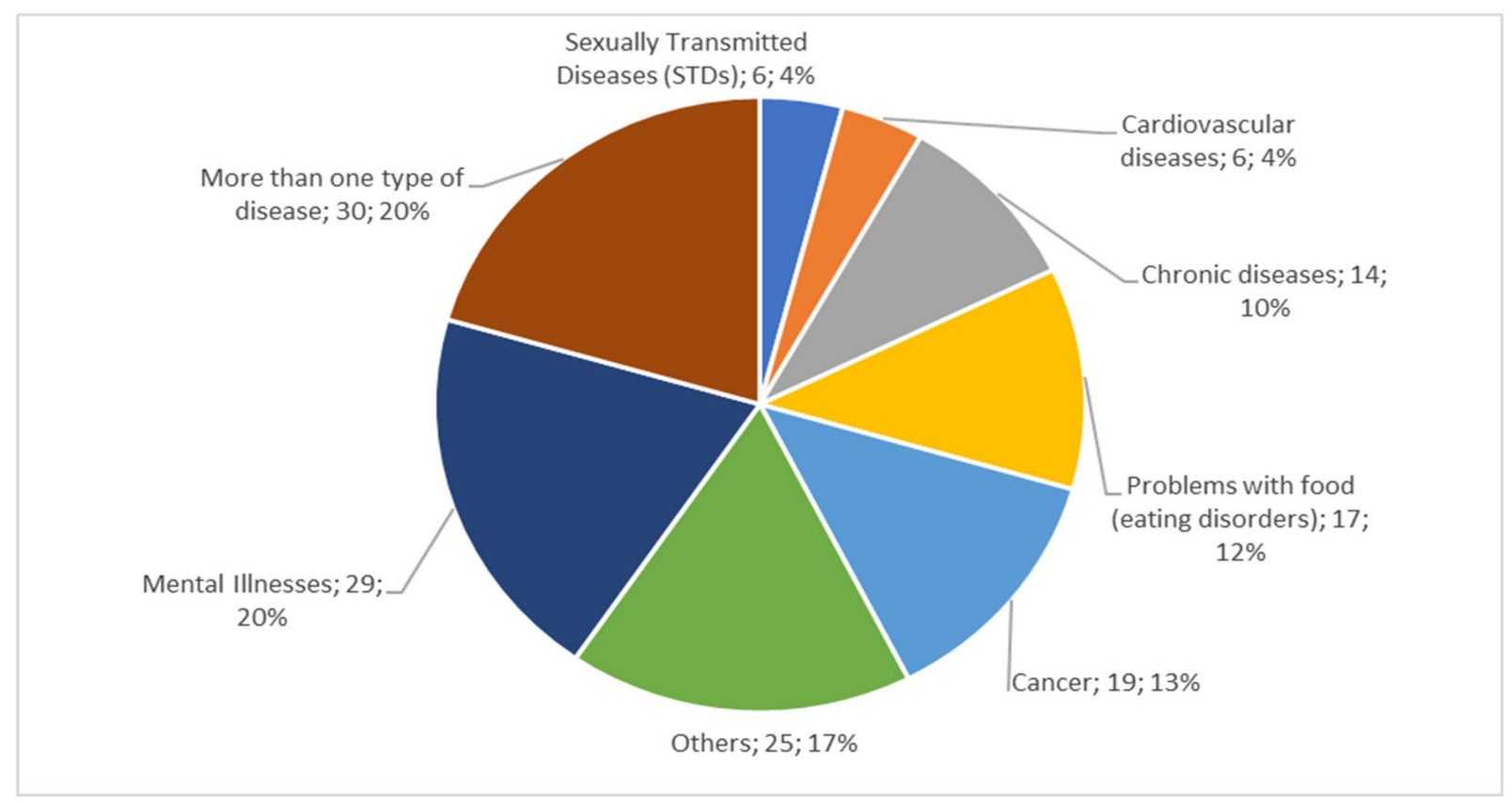

Figure 4. Diseases treated with e-health.

Some of the studies evaluate more than one type of issue, for instance, diabetes mellitus (DM), cardiovascular diseases, and chronic lung diseases [39]; mental illnesses such as depression, anxiety, dementia [76]; others, such as the oral health of pregnant women in prenatal care, anti-smoking, organ transplants [88]; cancer, such as breast cancer, skin cancer, and lung cancer [77]; eating disorders, including care regarding infant nutrition, diabetes, and obesity in adults [75]; chronic illness, such as chronic inflammatory rheumatic diseases (CIRDs), asthma, and chronic pain [10]; cardiovascular diseases [89]; cerebrovascular accident (CVA), coronary artery disease, and atrial fibrillation (AF) [90]; sexually transmitted diseases (STDs), such as HIV and/or sexually transmitted infections (STIs) [50]; human papillomavirus (HPV) and child sexual abuse [91].

Mental illnesses and the studies combining more than one type of disease represented $40 \%$ of diseases treated with e-health addressed in the literature. The studies deal mainly with randomized clinical trials applied to groups of people. For example, in the study conducted by [92], the authors verified how a telerehabilitation program works to help control type 2 DM. In the study by [93], a randomized clinical trial (RCT) was carried out with 13 primary brain tumor patients. Using the ReMind App, the pilot study obtained favorable results from patients but still required improvement. On the other hand, [94] observed 66 stroke (CVA) patients and their caregivers for eight weeks, using an e-health system as a treatment aid to do physical exercise. They found no significant effect on patient improvement during that period.

Other authors have chosen to review randomized studies, such as the study by [95], who compared the estimated sizes of combined effects through meta-analyses of analysis of covariance (ANCOVA), simple analysis of change score (SACS), and simple analysis of final values (SAFV), using RCTs of digital interventions with glycated hemoglobin $\mathrm{HbA1c}$ as the main result or intervention for disease treatment using e-health practices. Finally, in the study by [96], the use of mhealth for intervention as a form of treatment for patients with hallucinations was investigated. 


\subsubsection{Technologies Associated with E-Health}

From the final portfolio (446 articles), 57 publications were selected that address topics related to technology applied in healthcare. When answering the question "What are the most used technologies in e-health?" we identified IoT, cloud computing, Big Data, security, cryptography, algorithms, among others, as shown in Figure 5.

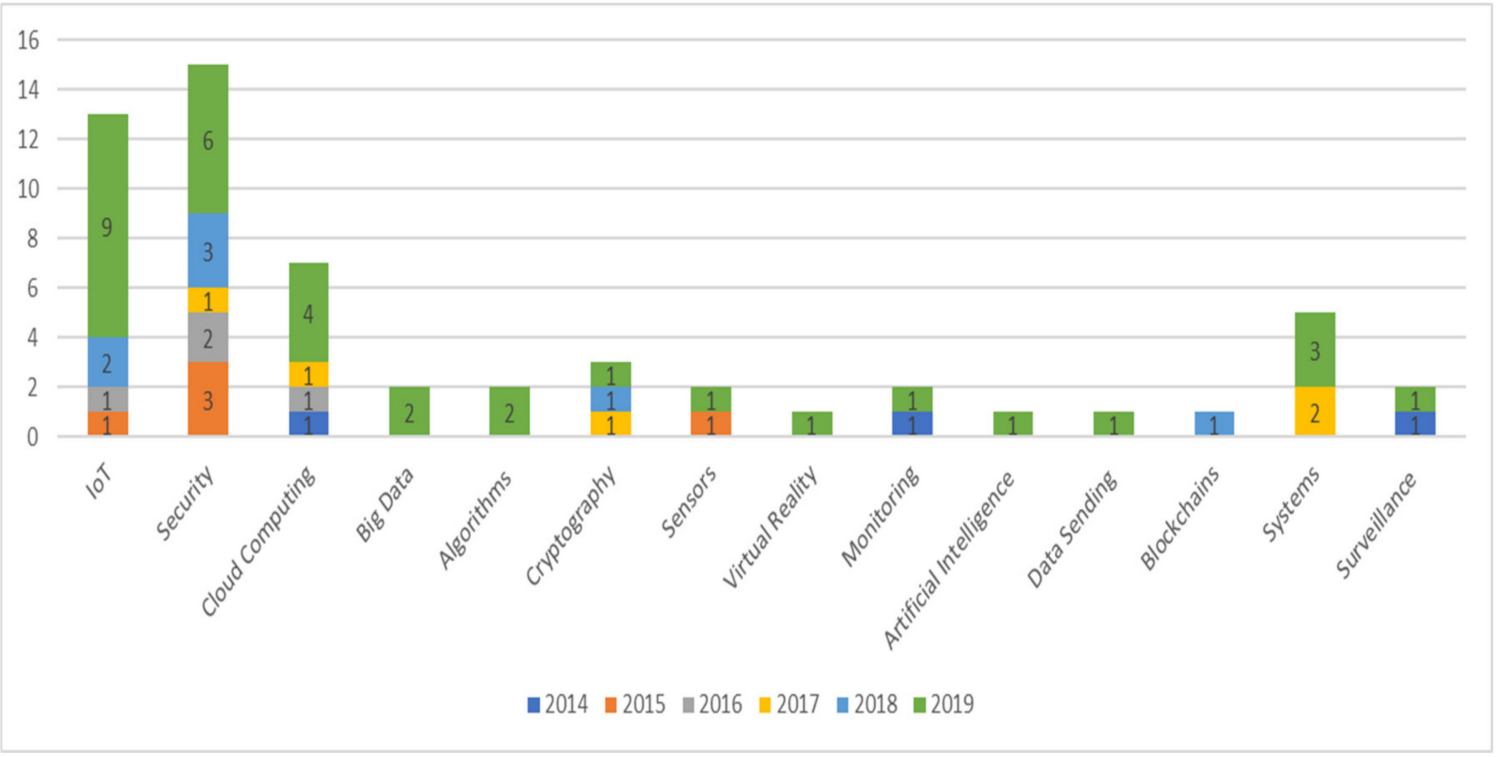

Figure 5. Number of publications in articles combining technology and e-health.

The technology applied to healthcare includes IoT to integrate medical sensors [80], authenticate, encrypt [97], and maintain security and confidentiality during data exchanges [98] and between physicians and patients [99]. It is vital that users are confident that the systems used are protected [100] and authenticated to ensure the privacy of stored data $[51,101]$. The cloud system facilitates data storage at a low cost while also being secure and private $[42,81,84]$.

The use of Big Data entails the analysis, diagnosis, and treatment of diseases [82,102]. There is also the use of wireless sensors applied to clothing, which can be used to monitor a patient when performing physical exercise [103-105].

The first authors of each of the 57 articles addressing technology in e-health are from 35 different countries, principally China (with 10 articles); India (6 articles); the United States, Australia, and Italy ( 3 articles each); and Greece, Algeria, Pakistan, and Saudi Arabia ( 2 articles each). Other countries, such as Spain, South Africa, and Mexico, had only one study. Articles related to technology were published in 38 journals, mainly Future Generation Computer Systems (7 articles); IEEE Access (4); International Journal of Information Management (4); Journal of Medical Systems (3); Computers \& Electrical Engineering (2); Health and Technology (2); Information Sciences (1); and Telematics and Informatics (1). In the literature review by [106] on the application of cloud computing in e-health, 44 studies published between 2010 and 2013 were analyzed. The authors were from countries including the United States (11), Australia (4), China (3), India (2), and Spain (1). As we can see, the results found by [106] are similar to those reported herein.

Through an analysis of the literature, we found that technology can provide benefits across healthcare practices, such as the development of products, processes, and systems, as well as support decision-making. Nonetheless, data security and privacy remain a concern, both for developers and users; regardless of how efficient technologies are becoming, there can always be failures. Thus, there is room for in-depth studies and the development of technologies with more secure systems. 


\section{Conclusions}

To achieve its proposed objective, the present study used a systematic literature review of articles published from 2014 to 2019 , resulting in the analysis of 446 articles. Six research questions were defined to analyze the literature on e-health practices. Through the analysis, four categories of the most common practices in the field were identified: "mhealth or mobile health"; "telehealth or telemedicine"; "technology"; and "others." The main services provided with e-health practices are health literacy, mhealth and monitoring, telehealth, and systems combined with technology. Regarding the fields of medical specialties that offer consultations via telehealth, the following were identified: telepsychiatry, teledermatology, telerehabilitation, teleophthalmology, telecardiology, and teledentistry. Other fields were also found that offer services in e-health, such as geriatrics, general medicine, endocrinology, pediatrics, gynecology, and oncology.

Furthermore, 146 of the 446 studies analyzed use one of the common practices for disease diagnosis/treatment/monitoring, with mental illnesses and studies combining the treatment of multiple disease corresponding to $40 \%$. Among the most frequently treated diseases are diabetes mellitus, cardiovascular diseases, chronic lung diseases, and mental illnesses (depression, anxiety, dementia). In terms of the countries that current use e-health, countries in Europe (Austria and Germany), Africa (Sub-Saharan countries), Asia (China), and North America (United States and Canada) stand out.

In relation to the technologies adopted in e-health practices, IoT, cloud computing, Big Data, security, and systems are among the most common.

Systemic reviews such as the one presented herein are important to provide more precise targeting of the practices and technologies that can be adopted in different procedures and processes, considering the context of each country. In general, such analyses can offer information and guide physicians and patients in the use of "new" services.

A study can always be improved, and the present one is no exception. Although the methodology applied followed careful steps and well-defined filters, the choice of only three keywords (considered comprehensive) may have resulted in the exclusion of some relevant studies in the analysis.

The present study was completed at the beginning of the global COVID-19 pandemic, during which physicians and patients began to use considerably more e-health practices and services due to the restrictions imposed by social isolation. Further studies are needed to address the growth in e-health practices, including services, diseases, and new approaches that are in greater demand, and the breakdown of government-imposed restrictions on expanding the use of e-health.

Author Contributions: All authors contributed in some way to the study design. M.H.d.F. wrote the article and had the help of F.K., C.T.P. guided and corrected the manuscript. B.P. made the final correction of the manuscript. P.R. helped in the correction of the manuscript. All authors have read and agreed to the published version of the manuscript.

Funding: This research was funded by the Coordenação de Aperfeiçoamento de Pessoal de Nível Superior-Brazil (CAPES)—Finance Code 001 and Fundação Araucária.

Informed Consent Statement: Not applicable.

Acknowledgments: The authors would like to thank the Organizations and Society Research Laboratory, Federal University of Technology-Paraná, Brazil.

Conflicts of Interest: The authors declare no conflict of interest. 


\section{Appendix A}

Table A1. Final portfolio.

\begin{tabular}{|c|c|c|c|c|c|c|}
\hline Author & Title & Journal & $\begin{array}{l}\text { Impact } \\
\text { Factor }\end{array}$ & Year & Citations & InOrdinatio 10 \\
\hline $\begin{array}{l}\text { Stoyanov, S.R.; Hides, L.; Kavanagh, } \\
\text { D.J.; Zelenko, O.; Tjondronegoro, D.; } \\
\text { Mani, M. }\end{array}$ & $\begin{array}{l}\text { Mobile app rating scale: A new tool for assessing } \\
\text { the quality of health mobile apps }\end{array}$ & JMIR $m$ Health and $u$ Health & 4.301 & 2015 & 550 & 550.00 \\
\hline $\begin{array}{l}\text { [38] Silva, B.M.C.; Rodrigues, J.J.P.C.; } \\
\text { Torre-Díez, I.D.L.; Lopez-Coronado, } \\
\text { M.; Saleem, K. }\end{array}$ & Mobile-health: A review of current state in 2015 & J. Biomed. Inf. & 2.950 & 2015 & 454 & 514.00 \\
\hline $\begin{array}{l}\text { [83] Flodgren, G.; Rachas, A.; Farmer, } \\
\text { A.J.; Inzitari, M.; Shepperd, S. }\end{array}$ & $\begin{array}{l}\text { Interactive telemedicine: Effects on professional } \\
\text { practice and health care outcomes }\end{array}$ & Cochrane Database Syst. Rev. & 7.755 & 2015 & 245 & 305.01 \\
\hline [39] Whitehead, L; Seaton, P. & $\begin{array}{l}\text { The effectiveness of self-management mobile } \\
\text { phone and tablet apps in long-term condition } \\
\text { management: A systematic review }\end{array}$ & J. Med. Internet. Res. & 4.945 & 2016 & 206 & 276.00 \\
\hline Sultan, N. & $\begin{array}{l}\text { Making use of cloud computing for healthcare } \\
\text { provision: Opportunities and challenges }\end{array}$ & $\begin{array}{l}\text { International Journal of Information } \\
\text { Management }\end{array}$ & 5.063 & 2014 & 202 & 252.01 \\
\hline $\begin{array}{l}\text { [105] Wang, J.B.; Cadmus-Bertram, } \\
\text { L.A.; Natarajan, L.; White, M.M.; } \\
\text { Madanat, H.; Nichols, J.F.; Ayala, G.X.; } \\
\text { Pierce, J.P. }\end{array}$ & $\begin{array}{c}\text { Wearable sensor/device (Fitbit One) and SMS } \\
\text { text-messaging prompts to increase physical } \\
\text { activity in overweight and obese adults: A } \\
\text { randomized controlled trial }\end{array}$ & Telemed, e-Health & 1.996 & 2015 & 173 & 233.00 \\
\hline $\begin{array}{l}\text { Fortino, G.; Galzarano, S.; Gravina, R.; } \\
\text { Li, W. }\end{array}$ & $\begin{array}{l}\text { A framework for collaborative computing and } \\
\text { multi-sensor data fusion in body sensor networks }\end{array}$ & Information Fusion & 10.716 & 2015 & 167 & 227.01 \\
\hline $\begin{array}{l}\text { Boudreaux, E.; Waring, M.; Hayes, R.; } \\
\text { Sadasivam, R.; Mullen, S.; Pagoto, S. }\end{array}$ & $\begin{array}{c}\text { Evaluating and selecting mobile health apps: } \\
\text { strategies for healthcare providers and healthcare } \\
\text { organizations }\end{array}$ & Transl. Behav. Med. & 2.237 & 2014 & 173 & 223.00 \\
\hline $\begin{array}{l}\text { [3] Ross, J.; Stevenson, F.; Lau, R.; } \\
\text { Murray, E. }\end{array}$ & $\begin{array}{l}\text { Factors that influence the implementation of } \\
\text { e-health: A systematic review of systematic } \\
\text { reviews (an update) }\end{array}$ & Implement Sci. & 0 & 2016 & 149 & 219.00 \\
\hline $\begin{array}{l}\text { Naslund, J.; Marsch, L.; McHugo, G.; } \\
\text { Bartels, S. }\end{array}$ & $\begin{array}{l}\text { Emerging mHealth and eHealth interventions for } \\
\text { serious mental illness: A review of the literature }\end{array}$ & J. Ment. Health & 2.604 & 2015 & 156 & 216.00 \\
\hline $\begin{array}{l}\text { [56] Torre-Díez, I.D.L.; } \\
\text { Lopez-Coronado, M.; Vaca, C.; Saez } \\
\text { Aguado, J.; De Castro, C. }\end{array}$ & $\begin{array}{l}\text { Cost-utility and cost-effectiveness studies of } \\
\text { telemedicine, electronic, and mobile health systems } \\
\text { in the literature: A Systematic review }\end{array}$ & Telemedicine and $\mathrm{e}$-Health & 1.996 & 2015 & 150 & 210.00 \\
\hline $\begin{array}{c}\text { Firth, J.; Torous, J.; Nicholas, J.; } \\
\text { Carney, R.; Rosenbaum, S.; Sarris, J. }\end{array}$ & $\begin{array}{l}\text { Can smartphone mental health interventions } \\
\text { reduce symptoms of anxiety? A meta-analysis of } \\
\text { randomized controlled trials }\end{array}$ & J. Affect Disord. & 4.084 & 2017 & 123 & 203.00 \\
\hline $\begin{array}{l}\text { Riley, R.D.; Ensor, J.; Snell, K.I.E.; } \\
\text { Debray, T.P.A.; Altman, D.G., Moons, } \\
\text { K.G.M.; Collins, G.S. }\end{array}$ & $\begin{array}{l}\text { External validation of clinical prediction models } \\
\text { using big datasets from e-health records or IPD } \\
\text { meta-analysis: Opportunities and challenges }\end{array}$ & BMJ—British Medical Journal & 27.604 & 2016 & 121 & 191.03 \\
\hline $\begin{array}{l}\text { Poria, S.; Cambria, E.; Hussain, A.; } \\
\text { Huang, G.B. }\end{array}$ & $\begin{array}{l}\text { Towards an intelligent framework for multimodal } \\
\text { affective data analysis }\end{array}$ & Neural Networks & 5.785 & 2015 & 131 & 191.01 \\
\hline $\begin{array}{l}\text { [74] Larsen, M.E.; Nicholas, J.; } \\
\text { Christensen, H. }\end{array}$ & $\begin{array}{l}\text { A systematic assessment of smartphone tools for } \\
\text { suicide prevention }\end{array}$ & Plos One & 2.776 & 2016 & 121 & 191.00 \\
\hline $\begin{array}{l}\text { [64] Hollis, C.; Falconer, C.; Martin, J.; } \\
\text { Whittington, C.; Stockton, S.; } \\
\text { Glazebrook, C.; Davies, E. }\end{array}$ & $\begin{array}{l}\text { Annual research review: digital health } \\
\text { interventions for children and young people with } \\
\text { mental health problems-a systematic and } \\
\text { meta-review }\end{array}$ & J. Child. Psychol. Psychiatry & 6.129 & 2017 & 109 & 189.01 \\
\hline Sultan, $\mathrm{N}$. & $\begin{array}{l}\text { Reflective thoughts on the potential and challenges } \\
\text { of wearable technology for healthcare provision } \\
\text { and medical education }\end{array}$ & $\begin{array}{l}\text { International Journal of Information } \\
\text { Management }\end{array}$ & 5.063 & 2015 & 114 & 174.01 \\
\hline $\begin{array}{l}\text { Aaronson, N.; Mattioli, V.; Minton, O.; } \\
\text { Weis, J.; Johansen, C.; Dalton, S.; } \\
\text { Verdonck-De Leeuw, L.; Stein, K.; } \\
\text { Alfano, C.; Mehnert, A.; De Boer, A.; } \\
\text { Van De Poll-Franse, L. }\end{array}$ & $\begin{array}{l}\text { Beyond treatment-psychosocial and behavioural } \\
\text { issues in cancer survivorship research and practice }\end{array}$ & Eur. J. Cancer & 6.680 & 2014 & 123 & 173.01 \\
\hline $\begin{array}{l}\text { Ben-Zeev, D.; Schueller, S.M.; Begale, } \\
\text { M.; Duffecy, J.; Kane, J.M.; Mohr, D.C. }\end{array}$ & $\begin{array}{l}\text { Strategies for mHealth research: lessons from } 3 \\
\text { mobile intervention studies }\end{array}$ & Adm. Policy. Ment. Health & 2.681 & 2015 & 111 & 171.00 \\
\hline $\begin{array}{l}\text { [80] Moosavi, S.R.; Gia, T.N.; Rahmani, } \\
\text { A.-M.; Nigussie, E.; Virtanen, S.; } \\
\text { Isoaho, J.; Tenhunen, H. }\end{array}$ & $\begin{array}{l}\text { SEA: A secure and efficient authentication and } \\
\text { authorization architecture for IoT-based healthcare } \\
\text { using smart gateways }\end{array}$ & Procedia Comput. Sci. & 0 & 2015 & 111 & 171.00 \\
\hline $\begin{array}{l}\text { Wagner, L.I.; Schink, J.; Bass, M.; Patel, } \\
\text { S.; Diaz, M.V.; Rothrock, N.; Pearman, } \\
\text { T.; Gershon, R.; Penedo, FJ.; Rosen, S.; } \\
\text { Cella, D. }\end{array}$ & $\begin{array}{l}\text { Bringing PROMIS to practice: Brief and precise } \\
\text { symptom screening in ambulatory cancer care }\end{array}$ & Cancer & 6.162 & 2015 & 110 & 170.01 \\
\hline Zhang, X.; Yu, P.; Yan, J.; Spil, I.T.A.M. & $\begin{array}{l}\text { Using diffusion of innovation theory to understand } \\
\text { the factors impacting patient acceptance and use of } \\
\text { consumer e-health innovations: A case study in a } \\
\text { primary care clinic }\end{array}$ & BMC Health Serv. Res. & 1.932 & 2015 & 110 & 170.00 \\
\hline $\begin{array}{l}\text { Ebert, D.; Berking, M.; Cuijpers, P.; } \\
\text { Lehr, D.; Pörtner, M.; Baumeister, H. }\end{array}$ & $\begin{array}{l}\text { Increasing the acceptance of internet-based mental } \\
\text { health interventions in primary care patients with } \\
\text { depressive symptoms. A randomized } \\
\text { controlled trial }\end{array}$ & J. Affect. Disord. & 4.084 & 2015 & 109 & 169.00 \\
\hline $\begin{array}{l}\text { Clifton, L.; Clifton, D.; Pimentel, M.; } \\
\text { Watkinson, P.; Tarassenko, L. }\end{array}$ & $\begin{array}{l}\text { Predictive monitoring of mobile patients by } \\
\text { combining clinical observations with data from } \\
\text { wearable sensors }\end{array}$ & IEEE J. Biomed. Health Inform. & 4.217 & 2014 & 118 & 168.00 \\
\hline $\begin{array}{l}\text { McKay, F.H.; Cheng, C.; Wright, A.; } \\
\text { Shill, J.; Stephens, H.; Uccellini, M. }\end{array}$ & $\begin{array}{l}\text { Evaluating mobile phone applications for health } \\
\text { behaviour change: A systematic review }\end{array}$ & J. Telemed. Telecare & 2.229 & 2018 & 78 & 168.00 \\
\hline Olff, M. & $\begin{array}{l}\text { Mobile mental health: A challenging } \\
\text { research agenda }\end{array}$ & Eur. J.Psychotraumatol & 3.020 & 2015 & 104 & 164.00 \\
\hline $\begin{array}{l}\text { [45] Wentzel, J.; Van Der Vaart, R.; } \\
\text { Bohlmeijer, E.T.; Van Gemert-Pijnen, } \\
\text { J.E.W.C. }\end{array}$ & $\begin{array}{l}\text { Mixing online and face-to-face therapy: How to } \\
\text { benefit from blended care in mental health care }\end{array}$ & JMIR Mental Health & 0 & 2016 & 93 & 163.00 \\
\hline [76] Grist, R.; Porter, J.; Stallard, P. & $\begin{array}{l}\text { Mental health mobile apps for preadolescents and } \\
\text { adolescents: A systematic review }\end{array}$ & J. Med. Internet Res. & 4.945 & 2017 & 82 & 162.00 \\
\hline
\end{tabular}


Table A1. Cont.

\begin{tabular}{|c|c|c|c|c|c|c|}
\hline Author & Title & Journal & $\begin{array}{l}\text { Impact } \\
\text { Factor }\end{array}$ & Year & Citations & InOrdinatio 10 \\
\hline Yüksel, B.; Küpçü, A.; Özkasap, O. & $\begin{array}{l}\text { Research issues for privacy and security of } \\
\text { electronic health services }\end{array}$ & Future Generation Computer Systems & 5.768 & 2017 & 77 & 157.01 \\
\hline $\begin{array}{l}\text { Martin, S.; Feldman, D.; Blumenthal, } \\
\text { R.; Jones, S.; Post, W.; McKibben, R.; } \\
\text { Michos, E.; Ndumele, C.; Ratchford, } \\
\text { E.; Coresh, J.; Blaha, M. }\end{array}$ & $\begin{array}{l}\text { mActive: A randomized clinical trial of an } \\
\text { automated mHealth intervention for physical } \\
\text { activity promotion }\end{array}$ & J. Am. Heart Assoc. & 4.660 & 2015 & 97 & 157.00 \\
\hline $\begin{array}{l}\text { Martínez-Pérez, B.; Torre-Díez, I.D.L.; } \\
\text { López-Coronado, M.; Sainz-De-Abajo, } \\
\text { B.; Robles, M.; García-Gómez, J. }\end{array}$ & $\begin{array}{l}\text { Mobile clinical decision support systems and } \\
\text { applications: A literature and commercial review }\end{array}$ & J. Med. Syst. & 2.415 & 2014 & 106 & 156.00 \\
\hline $\begin{array}{l}\text { Tang, J.; Abraham, C.; Stamp, E.; } \\
\text { Greaves, C. }\end{array}$ & $\begin{array}{l}\text { How can weight-loss app designers' best engage } \\
\text { and support users? A qualitative investigation }\end{array}$ & British Journal of Health Psychology & 2.472 & 2015 & 95 & 155.00 \\
\hline Alahäivälä, T.; Oinas-Kukkonen, H. & $\begin{array}{l}\text { Understanding persuasion contexts in health } \\
\text { gamification: A systematic analysis of gamified } \\
\text { health behavior change support systems literature }\end{array}$ & $\begin{array}{l}\text { International Journal of Medical } \\
\text { Informatics }\end{array}$ & 2.721 & 2016 & 83 & 153.00 \\
\hline Baker, T;; Gustafson, D.; Shah, D. & $\begin{array}{l}\text { How can research keep up with eHealth? Ten } \\
\text { strategies for increasing the timeliness and } \\
\text { usefulness of Ehealth research }\end{array}$ & J. Med. Internet Res. & 4.945 & 2014 & 102 & 152.00 \\
\hline $\begin{array}{l}\text { Konstantinidis, E.I.; Billis, A.S.; } \\
\text { Mouzakidis, C.A.; Zilidou, V.I.; } \\
\text { Antoniou, P.E.; Bamidis, P.D. }\end{array}$ & $\begin{array}{l}\text { Design, implementation, and wide pilot } \\
\text { deployment of FitForAll: An easy to use } \\
\text { exergaming platform improving physical fitness } \\
\text { and life quality of senior citizens }\end{array}$ & IEEE J. Biomed. Health Inform. & 4.217 & 2016 & 82 & 152.00 \\
\hline $\begin{array}{l}\text { Pramana, G.; Parmanto, B.; Kendall, } \\
\text { P.C.; Silk, J.S. }\end{array}$ & $\begin{array}{l}\text { The SmartCAT: An m-Health platform for } \\
\text { ecological momentary intervention in child } \\
\text { anxiety treatment }\end{array}$ & Telemedicine and $\mathrm{e}$-Health & 1.996 & 2014 & 102 & 152.00 \\
\hline Watkins, I.; Xie, B. & $\begin{array}{l}\text { eHealth literacy interventions for older adults: A } \\
\text { systematic review of the literature }\end{array}$ & J. Med. Internet Res. & 4.945 & 2014 & 101 & 151.00 \\
\hline $\begin{array}{l}\text { Kaipio, J.; Lääveri, T.; Hyppönen, H.; } \\
\text { Vainiomäki, S.; Reponen, J.; } \\
\text { Kushniruk, A.; Borycki, E.; Vänskä, J. }\end{array}$ & $\begin{array}{l}\text { Usability problems do not heal by themselves: } \\
\text { National survey on physicians' experiences with } \\
\text { EHRs in Finland }\end{array}$ & $\begin{array}{l}\text { International Journal of Medical } \\
\text { Informatics }\end{array}$ & 2.721 & 2017 & 70 & 150.00 \\
\hline $\begin{array}{l}\text { Ahmad, M.; Amin, M.B.; Hussain, S.; } \\
\text { Kang, B.H.; Cheong, T.; Lee, S. }\end{array}$ & $\begin{array}{l}\text { Health Fog: A novel framework for health and } \\
\text { wellness applications }\end{array}$ & Journal of Supercomputing & 2.157 & 2016 & 80 & 150.00 \\
\hline Kim, H.; Xie, B. & $\begin{array}{l}\text { Health literacy in the eHealth era: A systematic } \\
\text { review of the literature }\end{array}$ & Patient Educ. Couns. & 2.821 & 2017 & 68 & 148.00 \\
\hline $\begin{array}{l}\text { Wicks, P.; Stamford, J.; Grootenhuis, } \\
\text { M.; Haverman, L.; Ahmed, S. }\end{array}$ & Innovations in e-health & Qual. Life Res. & 2.488 & 2014 & 97 & 147.00 \\
\hline Gómez, J.; Oviedo, B.; Zhuma, E. & $\begin{array}{c}\text { Patient monitoring system based on Internet of } \\
\text { Things }\end{array}$ & Procedia Comput. Sci. & 0 & 2016 & 77 & 147.00 \\
\hline Cruz, J;; Brooks, D.; Marques, A. & $\begin{array}{l}\text { Home telemonitoring in COPD: A systematic } \\
\text { review of methodologies and patients' adherence }\end{array}$ & $\begin{array}{l}\text { International Journal of Medical } \\
\text { Informatics }\end{array}$ & 2.721 & 2014 & 96 & 146.00 \\
\hline $\begin{array}{l}\text { Hubley, S.; Lynch, S.B.; Schneck, C.; } \\
\text { Thomas, M.; Shore, J. }\end{array}$ & Review of key telepsychiatry outcomes & World J. Psychiatry & 0 & 2016 & 71 & 141.00 \\
\hline $\begin{array}{l}\text { Vegesna, A.; Tran, M.; Angelaccio, M.; } \\
\text { Arcona, S. }\end{array}$ & $\begin{array}{l}\text { Remote patient monitoring via non-invasive digital } \\
\text { technologies: A systematic review }\end{array}$ & Telemedicine and e-Health & 1.996 & 2017 & 60 & 140.00 \\
\hline $\begin{array}{l}\text { [37] Brinkel, J.; Krämer, A.; } \\
\text { Krumkamp, R.; May, J.; Fobil, J. }\end{array}$ & $\begin{array}{l}\text { Mobile phone-based mHealth approaches for } \\
\text { public health surveillance in sub-Saharan Africa: A } \\
\text { systematic review }\end{array}$ & Int. J. Environ. Res. Public Health & 2.468 & 2014 & 89 & 139.00 \\
\hline $\begin{array}{l}\text { [57] Kleiboer, A.; Smit, J.; Bosmans, J.; } \\
\text { Ruwaard, J.; Andersson, G.; Topooco, } \\
\text { N.; Berger, T.; Krieger, T.; Botella, C.; } \\
\text { Baños, R.; Chevreul, K; Araya, R.; } \\
\text { Cerga-Pashoja, A.; Cieślak, R.; Rogala, } \\
\text { A.; Vis, C.; Draisma, S.; Schaik, A.; } \\
\text { Kemmeren, L; Ebert, D.; Berking, M.; } \\
\text { Funk, B.; Cuijpers, P.; Riper, H. }\end{array}$ & $\begin{array}{l}\text { European COMPARative Effectiveness research on } \\
\text { blended Depression treatment versus } \\
\text { treatment-as-usual (E-COMPARED): Study } \\
\text { protocol for a randomized controlled, } \\
\text { non-inferiority trial in eight European countries }\end{array}$ & Trials & 1.975 & 2016 & 68 & 138.00 \\
\hline $\begin{array}{l}\text { De Veer, A.J.E.; Peeters, J.M.; Brabers, } \\
\text { A.E.M.; Schellevis, F.G.; Rademakers, } \\
\text { J.J.D.J.M.; Francke, A.L. }\end{array}$ & $\begin{array}{l}\text { Determinants of the intention to use e-health by } \\
\text { community dwelling older people }\end{array}$ & BMC Health Serv. Res. & 1.932 & 2015 & 78 & 138.00 \\
\hline $\begin{array}{l}\text { [58] Provoost, S.; Lau, H.M.; Ruwaard, } \\
\text { J;; Riper, H. }\end{array}$ & $\begin{array}{l}\text { Embodied conversational agents in clinical } \\
\text { psychology: A scoping review }\end{array}$ & J. Med. Internet Res. & 4.945 & 2017 & 57 & 137.00 \\
\hline Schueller, S.; Tomasino, K.; Mohr, D. & $\begin{array}{l}\text { Integrating human support into behavioral } \\
\text { intervention technologies: The efficiency model of } \\
\text { support }\end{array}$ & $\begin{array}{c}\text { Clinical Psychology: Science and } \\
\text { Practice }\end{array}$ & 0 & 2017 & 57 & 137.00 \\
\hline Latulippe, K.; Hamel, C.; Giroux, D. & $\begin{array}{l}\text { Social health inequalities and eHealth: A literature } \\
\text { review with qualitative synthesis of theoretical and } \\
\text { empirical studies }\end{array}$ & J. Med. Internet Res. & 4.945 & 2017 & 56 & 136.00 \\
\hline $\begin{array}{l}\text { Shaw, T.; McGregor, D.; Brunner, M.; } \\
\text { Keep, M.; Janssen, A.; Barnet, S. }\end{array}$ & $\begin{array}{l}\text { What is eHealth (6)? Development of a conceptual } \\
\text { model for eHealth: Qualitative study with } \\
\text { key informants }\end{array}$ & J. Med. Internet Res. & 4.945 & 2017 & 56 & 136.00 \\
\hline [49] Pearce, C.; Bainbridge, M. & $\begin{array}{l}\text { A personally controlled electronic health record } \\
\text { for Australia }\end{array}$ & J. Am. Med. Inform. Assoc. & 4.292 & 2014 & 86 & 136.00 \\
\hline $\begin{array}{l}\text { Levack, W.; Weatherall, M.; } \\
\text { Hay-Smith, E.; Dean, S.; Mcpherson, } \\
\text { K.; Siegert, R. }\end{array}$ & $\begin{array}{l}\text { Goal setting and strategies to enhance goal pursuit } \\
\text { for adults with acquired disability participating } \\
\text { in rehabilitation }\end{array}$ & $\begin{array}{c}\text { Cochrane Database of Systematic } \\
\text { Reviews }\end{array}$ & 7.755 & 2015 & 75 & 135.01 \\
\hline $\begin{array}{l}\text { [90] Brieger, D;; Amerena, J.; Attia, J; } \\
\text { Bajorek, B; Chan, K.; Connell, C.; } \\
\text { Freedman, B.; Ferguson, C.; Hall, T.; } \\
\text { Haqqani, H.; Hendriks, J.; Hespe, C.; } \\
\text { Hung, J.; Kalman, J.; } \\
\text { Wonders, P.; } \\
\text { Worthington, J.; Yan, T.; Zwar, N. }\end{array}$ & $\begin{array}{l}\text { National Heart Foundation of Australia and } \\
\text { Cardiac Society of Australia and New Zealand: } \\
\text { Australian clinical guidelines for the diagnosis and } \\
\text { management of atrial fibrillation } 2018\end{array}$ & Med. J. Aust. & 0 & 2018 & 45 & 135.00 \\
\hline Brian, R.M.; Ben-Zeev, D. & $\begin{array}{l}\text { Mobile health (mHealth) for mental health in Asia: } \\
\text { Objectives, strategies, and limitations }\end{array}$ & Asian Journal of Psychiatry & 1.932 & 2014 & 83 & 133.00 \\
\hline $\begin{array}{l}\text { Ebert, D.D.; Cuijpers, P.; Munoz, R.F.; } \\
\text { Baumeister, H. }\end{array}$ & $\begin{array}{l}\text { Prevention of mental health disorders using } \\
\text { internet-and mobile-based interventions: A } \\
\text { narrative review and recommendations for } \\
\text { future research }\end{array}$ & Front Psychiatry & 3.161 & 2017 & 52 & 132.00 \\
\hline
\end{tabular}


Table A1. Cont.

\begin{tabular}{|c|c|c|c|c|c|c|}
\hline Author & Title & Journal & $\begin{array}{l}\text { Impact } \\
\text { Factor }\end{array}$ & Year & Citations & InOrdinatio 10 \\
\hline $\begin{array}{l}\text { [55] Glasgow, R.E.; Phillips, S.M.; } \\
\text { Sanchez, M.A. }\end{array}$ & $\begin{array}{l}\text { Implementation science approaches for integrating } \\
\text { eHealth research into practice and policy }\end{array}$ & Int. J. Med. Inform. & 2.721 & 2014 & 82 & 132.00 \\
\hline $\begin{array}{l}\text { [50] Bauermeister, J.; Pingel, E.; } \\
\text { Jadwin-Cakmak, L.; Harper, G., } \\
\text { Horvath, K.; Weiss, G.; Dittus, P. }\end{array}$ & $\begin{array}{l}\text { Acceptability and preliminary efficacy of a tailored } \\
\text { online HIV/STI testing intervention for young men } \\
\text { who have sex with men: The Get } \\
\text { Connected! program }\end{array}$ & AIDS Behav. & 2.908 & 2015 & 70 & 130.00 \\
\hline Daniel, H.; Sulmasy, L.S. & $\begin{array}{l}\text { Policy recommendations to guide the use of } \\
\text { telemedicine in primary care settings: An } \\
\text { American College of Physicians position paper }\end{array}$ & Annals of Internal Medicine & 9.315 & 2015 & 68 & 128.01 \\
\hline $\begin{array}{l}\text { Kazemi, D.M.; Borsari, B.; Levine, } \\
\text { M.J., Li, S.; Lamberson, K.A.; } \\
\text { Matta, L.A. }\end{array}$ & $\begin{array}{l}\text { A systematic review of the mHealth interventions } \\
\text { to prevent alcohol and substance abuse }\end{array}$ & Journal of Health Communication & 1.773 & 2017 & 48 & 128.00 \\
\hline $\begin{array}{l}\text { [62] Rotondi, A.J.; Eack, S.M.; Hanusa, } \\
\text { B.H.; Spring, M.B.; Haas, G.L. }\end{array}$ & $\begin{array}{l}\text { Critical design elements of e-health applications for } \\
\text { users with severe mental illness: Singular focus, } \\
\text { simple architecture, prominent contents, explicit } \\
\text { navigation, and inclusive hyperlinks }\end{array}$ & Schizophr. Bull. & 7.289 & 2015 & 67 & 127.01 \\
\hline $\begin{array}{l}\text { Mohr, D.; Lyon, A.; Lattie, E.; Reddy, } \\
\text { M.; Schueller, S. }\end{array}$ & $\begin{array}{l}\text { Accelerating digital mental health research from } \\
\text { early design and creation to successful } \\
\text { implementation and sustainment }\end{array}$ & J. Med. Internet Res. & 4.945 & 2017 & 47 & 127.00 \\
\hline $\begin{array}{l}\text { Kumanyika, S.; Whitt-Glover, M.; } \\
\text { Haire-Joshu, D. }\end{array}$ & $\begin{array}{l}\text { What works for obesity prevention and treatment } \\
\text { in black Americans? Research directions }\end{array}$ & Obes. Rev. & 8.192 & 2014 & 75 & 125.01 \\
\hline $\begin{array}{l}\text { Mooney, K.; Beck, S.; Wong, B.; } \\
\text { Dunson, W.; Wujcik, D.; Whisenant, } \\
\text { M.; Donaldson, G. }\end{array}$ & $\begin{array}{l}\text { Automated home monitoring and management of } \\
\text { patient-reported symptoms during chemotherapy: } \\
\text { Results of the symptom care at home RCT }\end{array}$ & Cancer Med. & 3.357 & 2017 & 45 & 125.00 \\
\hline Zhang, A.; Lin, X. & $\begin{array}{l}\text { Towards secure and privacy-preserving data } \\
\text { sharing in e-health systems via } \\
\text { consortium blockchain }\end{array}$ & J. Med. Syst. & 2.415 & 2018 & 34 & 124.00 \\
\hline $\begin{array}{l}\text { Devlin, A.M.; McGee-Lennon, M.; } \\
\text { O'Donnell, C.A.; Bouamrane, M.-M.; } \\
\text { Agbakoba, R.; O'Connor, S.; Grieve, } \\
\text { E.; Finch, T.; Wyke, S., Watson, N.; } \\
\text { Browne, S.; Mair, F.S.; Team, D.E. }\end{array}$ & $\begin{array}{l}\text { Delivering digital health and well-being at scale: } \\
\text { Lessons learned during the implementation of the } \\
\text { Dallas program in the United Kingdom }\end{array}$ & J. Am. Med. Inform. Assoc. & 4.292 & 2016 & 53 & 123.00 \\
\hline $\begin{array}{l}\text { Luna, D.; Almerares, A.; Mayan III, J.; } \\
\text { De Quirós, F; Otero, C. }\end{array}$ & $\begin{array}{l}\text { Health informatics in developing countries: Going } \\
\text { beyond pilot practices to sustainable } \\
\text { implementations: A review of the } \\
\text { current challenges }\end{array}$ & Healthcare Informatics Research & 0 & 2014 & 73 & 123.00 \\
\hline $\begin{array}{l}\text { Finnane, A.; Dallest, K.; Janda, M.; } \\
\text { Soyer, H.P. }\end{array}$ & $\begin{array}{l}\text { Teledermatology for the diagnosis and } \\
\text { management of skin cancer: A systematic review }\end{array}$ & JAMA Dermatol. & 7.995 & 2017 & 42 & 122.01 \\
\hline $\begin{array}{l}\text { [63] Jackson, B.; Gray, K.; Knowlesd, } \\
\text { S.; De Cruz, P. }\end{array}$ & $\begin{array}{l}\text { EHealth technologies in inflammatory bowel } \\
\text { disease: A systematic review }\end{array}$ & Journal of Crohn's and Colitis & 7.827 & 2016 & 52 & 122.01 \\
\hline $\begin{array}{l}\text { Tinschert, P.; Jakob, R.; Barata, F.; } \\
\text { Kramer, J.N.; Kowatsch, T. }\end{array}$ & $\begin{array}{l}\text { The potential of mobile apps for improving asthma } \\
\text { self-management: A review of publicly available } \\
\text { and well-adopted asthma apps }\end{array}$ & JMIR $m$ Health and $u$ Health & 4.301 & 2017 & 42 & 122.00 \\
\hline $\begin{array}{l}\text { Hilty, D.; Crawford, A.; Teshima, J.; } \\
\text { Chan, S.; Sunderji, N.; Yellowlees, P.; } \\
\text { Kramer, G.; O'Neill, P.; Fore, C., Luo, } \\
\text { J.; Li, S.T. }\end{array}$ & $\begin{array}{l}\text { A framework for telepsychiatric training and } \\
\text { e-health: Competency-based education, evaluation } \\
\text { and implications }\end{array}$ & International Review of Psychiatry & 2.991 & 2015 & 62 & 122.00 \\
\hline $\begin{array}{l}\text { [73] Diviani, N.; Van Den Putte, B.; } \\
\text { Meppelink, C.S.; Van Weert, J.C. }\end{array}$ & $\begin{array}{l}\text { Exploring the role of health literacy in the } \\
\text { evaluation of online health information: Insights } \\
\text { from A mixed-methods study }\end{array}$ & Patient Educ. Couns. & 2.821 & 2016 & 52 & 122.00 \\
\hline $\begin{array}{l}\text { [6] Van Houwelingen, C.; Moerman, } \\
\text { A.; Ettema, R.; Kort, H.; Ten Cate, O. }\end{array}$ & $\begin{array}{l}\text { Competencies required for nursing telehealth } \\
\text { activities: A Delphi-study }\end{array}$ & Nurse Educ. Today & 2.442 & 2016 & 52 & 122.00 \\
\hline $\begin{array}{l}\text { Molini-Avejonas, D.R.; Rondon-Melo, } \\
\text { S.; Amato, C.A.D.L.H.; } \\
\text { Samelli, A.G. }\end{array}$ & $\begin{array}{l}\text { A systematic review of the use of telehealth in } \\
\text { speech, language and hearing sciences }\end{array}$ & J. Telemed Telecare & 2.229 & 2015 & 62 & 122.00 \\
\hline $\begin{array}{l}\text { Chauhan, D.S.; Singh, A.K.; Kumar, B.; } \\
\text { Saini, J.P. }\end{array}$ & $\begin{array}{l}\text { Quantization based multiple medical information } \\
\text { watermarking for secure e-health }\end{array}$ & Multimedia Tools and Applications & 2.101 & 2019 & 22 & 122.00 \\
\hline $\begin{array}{l}\text { Bradford, N.K.; Caffery, L.J.; Smith, } \\
\text { A.C. }\end{array}$ & $\begin{array}{l}\text { Telehealth services in rural and remote Australia: A } \\
\text { systematic review of models of care and factors } \\
\text { influencing success and sustainability }\end{array}$ & Rural Remote Health & 0.985 & 2016 & 52 & 122.00 \\
\hline $\begin{array}{l}\text { Townsend, A.; Leese, J.; Adam, P.; } \\
\text { McDonald, M.; Li, LC.; Kerr, S.; } \\
\text { Backman, C.L. }\end{array}$ & $\begin{array}{l}\text { eHealth, participatory medicine, and ethical care: A } \\
\text { focus group study of patients' and health care } \\
\text { providers' use of health-related } \\
\text { internet information }\end{array}$ & J. Med. Internet Res. & 4.945 & 2015 & 61 & 121.00 \\
\hline $\begin{array}{l}\text { Tensen, E.; Van Der Heijden, J.; } \\
\text { Jaspers, M.; Witkamp, L. }\end{array}$ & $\begin{array}{l}\text { Two decades of teledermatology: Current status } \\
\text { and integration in national healthcare systems }\end{array}$ & Curr Dermatol Rep. & 0 & 2016 & 51 & 121.00 \\
\hline $\begin{array}{l}\text { Andreassen, H.K.; Kjekshus, L.E.; } \\
\text { Tjora, A. }\end{array}$ & $\begin{array}{l}\text { Survival of the project: A case study of ICT } \\
\text { innovation in health care }\end{array}$ & Soc. Sci. Med. & 3.087 & 2015 & 60 & 120.00 \\
\hline $\begin{array}{l}\text { [75] Malasinghe, L.P.; Ramzan, N.; } \\
\text { Dahal, K. }\end{array}$ & $\begin{array}{l}\text { Remote patient monitoring: A } \\
\text { comprehensive study }\end{array}$ & $\begin{array}{l}\text { J. Ambient. Intell. Humaniz. } \\
\text { Comput. }\end{array}$ & 1.910 & 2019 & 20 & 120.00 \\
\hline $\begin{array}{l}\text { Shen, Q.; Liang, X.; Shen, X.S.; Lin, X.; } \\
\text { Luo, H.Y. }\end{array}$ & $\begin{array}{l}\text { Exploiting geo-distributed clouds for a e-health } \\
\text { monitoring system with minimum service delay } \\
\text { and privacy preservation }\end{array}$ & IEEE J. Biomed. Health Inform. & 4.217 & 2014 & 69 & 119.00 \\
\hline $\begin{array}{l}\text { Vinding, K.; Elsberg, H.; Thorkilgaard, } \\
\text { T.; Belard, E.; Pedersen, N.; Elkjaer, M.; } \\
\text { Marker, D.; Carlsen, K.; Burisch, J.; } \\
\text { Munkholm, P. }\end{array}$ & $\begin{array}{l}\text { Fecal calprotectin measured by patients at home } \\
\text { using smartphones-A new clinical tool in } \\
\text { monitoring patients with inflammatory } \\
\text { bowel disease }\end{array}$ & Inflammatory Bowel Diseases & 4.005 & 2016 & 49 & 119.00 \\
\hline Abdmeziem, M.R.; Tandjaoui, D. & $\begin{array}{l}\text { An end-to-end secure key management protocol } \\
\text { for e-health applications }\end{array}$ & Computers \& Electrical Engineering & 2.189 & 2015 & 59 & 119.00 \\
\hline $\begin{array}{l}\text { [77] Lubberding, S.; Van Uden-Kraan, } \\
\text { C.; Te Velde, E.; Cuijpers, P., Leemans, } \\
\text { C.; Verdonck-de Leeuw, I. }\end{array}$ & $\begin{array}{l}\text { Improving access to supportive cancer care } \\
\text { through an eHealth application: A qualitative } \\
\text { needs assessment among cancer survivors }\end{array}$ & J. Clin. Nurs. & 1.757 & 2015 & 59 & 119.00 \\
\hline Leaman, R.; Khare, R.; Lu, Z. & $\begin{array}{l}\text { Challenges in clinical natural language processing } \\
\text { for automated disorder normalization }\end{array}$ & J. Biomed. Inf. & 2.950 & 2015 & 58 & 118.00 \\
\hline
\end{tabular}


Table A1. Cont.

\begin{tabular}{|c|c|c|c|c|c|c|}
\hline Author & Title & Journal & $\begin{array}{l}\text { Impact } \\
\text { Factor }\end{array}$ & Year & Citations & InOrdinatio 10 \\
\hline Chung, S.Y.; Nahm, E.S. & $\begin{array}{l}\text { Testing reliability and validity of the eHealth } \\
\text { Literacy Scale (eHEALS) for older adults } \\
\text { recruited online }\end{array}$ & $\begin{array}{l}\text { CIN-Computers Informatics } \\
\text { Nursing }\end{array}$ & 1.029 & 2015 & 58 & 118.00 \\
\hline $\begin{array}{l}\text { Iqbal, S.; Kiah, M.L.M.; Zaidan, A.A.; } \\
\text { Zaidan, B.B.; Albahri, O.S.; Albahri, } \\
\text { A.S.; Alsalem, M.A. }\end{array}$ & $\begin{array}{l}\text { Real-time-based e-health systems: Design and } \\
\text { implementation of a lightweight key management } \\
\text { protocol for securing sensitive information } \\
\text { of patients }\end{array}$ & Health and Technology & 0 & 2019 & 18 & 118.00 \\
\hline East, M.L.; Havard, B.C. & $\begin{array}{l}\text { Mental health mobile apps: From infusion to } \\
\text { diffusion in the mental health social system }\end{array}$ & JMIR Mental Health & 0 & 2015 & 58 & 118.00 \\
\hline $\begin{array}{l}\text { [79] Domingues, M.; Alberto, N.; } \\
\text { Leitão, C.; Tavares, C.; De Lima, E.; } \\
\text { Radwan, A.; Sucasas, V.; Rodriguez, J.; } \\
\text { Andre, P.; Antunes, P. }\end{array}$ & $\begin{array}{l}\text { Insole optical fiber sensor architecture for remote } \\
\text { gait analysis-an e-health solution }\end{array}$ & IEEE Internet Things J. & 9.515 & 2019 & 17 & 117.01 \\
\hline $\begin{array}{l}\text { [40] Baumel, A.; Faber, K.; Mathur, N.; } \\
\text { Kane, J; Muench, F. }\end{array}$ & $\begin{array}{l}\text { Enlight: A comprehensive quality and therapeutic } \\
\text { potential evaluation tool for mobile and web-based } \\
\text { eHealth interventions }\end{array}$ & J. Med. Internet Res. & 4.945 & 2017 & 37 & 117.00 \\
\hline Zhang, L.; Zhu, S.; Tang, S. & $\begin{array}{l}\text { Privacy protection for telecare medicine } \\
\text { information systems using a chaotic map-based } \\
\text { three-factor authenticated key agreement scheme }\end{array}$ & IEEE J. Biomed. Health Inform. & 4.217 & 2017 & 37 & 117.00 \\
\hline $\begin{array}{l}\text { [104] Zhou, J.; Cao, Z.; Dong, X.; } \\
\text { Lin, X. }\end{array}$ & $\begin{array}{l}\text { Security and privacy in cloud-assisted wireless } \\
\text { wearable communications: Challenges, solutions } \\
\text { and, future directions }\end{array}$ & IEEE Wirel. Commun. & 3.546 & 2015 & 57 & 117.00 \\
\hline $\begin{array}{l}\text { Maguire, R.; Ream, E.; Richardson, A.; } \\
\text { Connaghan, J.; Johnston, B.; } \\
\text { Kotronoulas, G.; Pedersen, V.; } \\
\text { McPhelim, J.; Pattison, N.; Smith, A.; } \\
\text { Webster, L.; Taylor, A.; Kearney, N. }\end{array}$ & $\begin{array}{l}\text { Development of a novel remote patient monitoring } \\
\text { system: The advanced symptom management } \\
\text { system for radiotherapy to improve the symptom } \\
\text { experience of patients with lung cancer } \\
\text { receiving radiotherapy }\end{array}$ & Cancer Nursing & 2.022 & 2015 & 57 & 117.00 \\
\hline Koivunen, M.; Saranto, K. & $\begin{array}{l}\text { Nursing professionals' experiences of the } \\
\text { facilitators and barriers to the use of telehealth } \\
\text { applications: A systematic review of } \\
\text { qualitative studies }\end{array}$ & Scand. J. Caring. Sci. & 1.642 & 2018 & 27 & 117.00 \\
\hline Thomas, J.; Bond, D. & $\begin{array}{l}\text { Review of innovations in digital health technology } \\
\text { to promote weight control }\end{array}$ & Curr. Diab. Rep. & 0 & 2014 & 67 & 117.00 \\
\hline $\begin{array}{l}\text { [7] Jhamb, M.; Cavanaugh, K.L.; Bian, } \\
\text { A.; Chen, G.; Ikizler, T.A.; Unruh, } \\
\text { M.L.; Abdel-Kader, K. }\end{array}$ & $\begin{array}{l}\text { Disparities in electronic health record patient portal } \\
\text { use in nephrology clinics }\end{array}$ & Clin. J. Am. Soc. Nephrol. & 6.243 & 2015 & 56 & 116.01 \\
\hline $\begin{array}{l}\text { [72] Mackert, M.; Champlin, S.E.; } \\
\text { Holton, A.; Munoz, I.I.; Damasio, M.J. }\end{array}$ & $\begin{array}{l}\text { eHealth and health literacy: A research } \\
\text { methodology review }\end{array}$ & J Comput. - Mediat. Commun. & 4.896 & 2014 & 66 & 116.00 \\
\hline $\begin{array}{l}\text { Berrouiguet, S.; Perez-Rodriguez, } \\
\text { M.M.; Larsen, M.; ;aca-Garcia, E., } \\
\text { Courtet, P.; Oquendo, M. } \\
\end{array}$ & $\begin{array}{l}\text { From eHealth to iHealth: Transition to } \\
\text { participatory and personalized medicine in } \\
\text { mental health }\end{array}$ & J. Med. Internet Res. & 4.945 & 2018 & 25 & 115.00 \\
\hline $\begin{array}{l}\text { Grönloh, C.; Myreteg, G.; Cajander, } \\
\text { A.; Rexhepi, H. }\end{array}$ & $\begin{array}{l}\text { "Why do they need to check me?" Patient } \\
\text { participation through ehealth and the } \\
\text { doctor-patient relationship: qualitative study }\end{array}$ & J. Med. Internet Res. & 4.945 & 2018 & 25 & 115.00 \\
\hline $\begin{array}{l}\text { Rouleau, G.; Gagnon, M.P.; Côté, J.; } \\
\text { Payne-Gagnon, J.; Hudson, E.; } \\
\text { Dubois, C.-A. }\end{array}$ & $\begin{array}{c}\text { Impact of information and communication } \\
\text { technologies on nursing care: Results of an } \\
\text { overview of systematic reviews }\end{array}$ & J. Med. Internet Res. & 4.945 & 2017 & 35 & 115.00 \\
\hline $\begin{array}{l}\text { Lie, S.; Karlsen, B.; Oord, E.; Graue, } \\
\text { M.; Oftedal, B. }\end{array}$ & $\begin{array}{l}\text { Dropout from an eHealth intervention for adults } \\
\text { with type } 2 \text { diabetes: A qualitative study }\end{array}$ & J. Med. Internet Res. & 4.945 & 2017 & 35 & 115.00 \\
\hline $\begin{array}{l}\text { Venkatesh, V.; Rai, A.; Sykes, T.; } \\
\text { Aljafari, R. }\end{array}$ & $\begin{array}{l}\text { Combating infant mortality in rural India: } \\
\text { Evidence from a field study of Ehealth } \\
\text { kiosk implementations }\end{array}$ & Mis $Q$. & 4.373 & 2016 & 45 & 115.00 \\
\hline $\begin{array}{l}\text { Christensen, H.; Batterham, P.; } \\
\text { O'Dea, B. }\end{array}$ & E-health interventions for suicide prevention & Int. J. Environ. Res. Public Health & 2.468 & 2014 & 65 & 115.00 \\
\hline Uscher-Pines, L;; Mehrotra, A. & $\begin{array}{l}\text { Analysis of Teladoc use seems to indicate } \\
\text { expanded access to care for patients without prior } \\
\text { connection to a provider }\end{array}$ & Health Aff. (Millwood) & 0 & 2014 & 65 & 115.00 \\
\hline $\begin{array}{l}\text { [52] Wang, X.A.; Ma, J.; Xhafa, F.; } \\
\text { Zhang, M.; Luo, X. }\end{array}$ & $\begin{array}{l}\text { Cost-effective secure E-health cloud system using } \\
\text { identity based cryptographic techniques }\end{array}$ & Future Gener. Comp. Syst. & 5.768 & 2017 & 34 & 114.01 \\
\hline $\begin{array}{l}\text { Meri, A.; Hasan, M.; Danaee, M.; } \\
\text { Jaber, M.; Jarrar, M.; Safei, N.; } \\
\text { Dauwed, M.; Abd, S.K.; Al-bsheish M. }\end{array}$ & $\begin{array}{l}\text { Modelling the utilization of cloud health } \\
\text { information systems in the Iraqi public } \\
\text { healthcare sector }\end{array}$ & Telematics and Informatics & 3.714 & 2019 & 14 & 114.00 \\
\hline $\begin{array}{l}\text { Handayani, P.W.; Hidayanto, A.N.; } \\
\text { Pinem, A.A.; Hapsari, I.C.; } \\
\text { Sandhyaduhita, P.I.; Budi, I. }\end{array}$ & Acceptance model of a hospital information system & $\begin{array}{l}\text { International Journal of Medical } \\
\text { Informatics }\end{array}$ & 2.721 & 2017 & 34 & 114.00 \\
\hline $\begin{array}{l}\text { Kampmeijer, R.; Pavlova, M.; Tambor, } \\
\text { M.; Golinowska, S.; Groot, W. }\end{array}$ & $\begin{array}{l}\text { The use of e-health and m-health tools in health } \\
\text { promotion and primary prevention among older } \\
\text { adults: A systematic literature review }\end{array}$ & BMC Health Serv Res & 1.932 & 2016 & 44 & 114.00 \\
\hline [53] Romanou, A. & $\begin{array}{l}\text { The necessity of the implementation of Privacy by } \\
\text { Design in sectors where data protection } \\
\text { concerns arise }\end{array}$ & Computer Law \& Security Review & 1.552 & 2018 & 24 & 114.00 \\
\hline Hoque, M.R.; Bao, Y.; Sorwarb, G. & $\begin{array}{l}\text { Investigating factors influencing the adoption of } \\
\text { e-health in developing countries: A } \\
\text { patient's perspective }\end{array}$ & Inform. Health Soc. Care & 1.218 & 2017 & 34 & 114.00 \\
\hline Zhang, L.; Zhang, Y.; Tang, S.; Luo, H. & $\begin{array}{l}\text { Privacy protection for e-health systems by means } \\
\text { of dynamic authentication and three-factor } \\
\text { key agreement }\end{array}$ & IEEE Trans. Ind. Electron. & 7.503 & 2018 & 23 & 113.01 \\
\hline $\begin{array}{l}\text { [42] García, L.; Tomás, J; Parra, L.; } \\
\text { Lloret, J. }\end{array}$ & $\begin{array}{l}\text { An m-health application for cerebral stroke } \\
\text { detection and monitoring using cloud services }\end{array}$ & Inf. Int. J. Manag. & 5.063 & 2019 & 13 & 113.01 \\
\hline $\begin{array}{l}\text { Greenhalgh, T.; Stones, R.; } \\
\text { Swinglehurst, D. }\end{array}$ & $\begin{array}{l}\text { Choose and book: A sociological analysis of } \\
\text { "resistance" to an expert system }\end{array}$ & Soc. Sci. Med. & 3.087 & 2014 & 63 & 113.00 \\
\hline $\begin{array}{l}\text { Giunti, G.; Giunta, D.; } \\
\text { Guisado-Fernandez, E.; Bender, J.; } \\
\text { Fernandez-Luque, L. }\end{array}$ & $\begin{array}{l}\text { A biopsy of breast cancer mobile applications: State } \\
\text { of the practice review }\end{array}$ & $\begin{array}{l}\text { International Journal of Medical } \\
\text { Informatics }\end{array}$ & 2.721 & 2018 & 23 & 113.00 \\
\hline
\end{tabular}


Table A1. Cont.

\begin{tabular}{|c|c|c|c|c|c|c|}
\hline Author & Title & Journal & $\begin{array}{l}\text { Impact } \\
\text { Factor }\end{array}$ & Year & Citations & InOrdinatio 10 \\
\hline $\begin{array}{l}\text { Bujnowska-Fedak, M.M.; } \\
\text { Pirogowicz, I. }\end{array}$ & $\begin{array}{l}\text { Support for e-health services among elderly } \\
\text { primary care patients }\end{array}$ & Telemedicine and e-Health & 1.996 & 2014 & 63 & 113.00 \\
\hline $\begin{array}{l}\text { Meurk, C.; Leung, J.; Hall, W.; Head, } \\
\text { B.W.; Whiteford, H. }\end{array}$ & $\begin{array}{l}\text { Establishing and governing e-mental health care in } \\
\text { Australia: A systematic review of challenges and a } \\
\text { call for policy-focussed research }\end{array}$ & J. Med. Internet Res. & 4.945 & 2016 & 42 & 112.00 \\
\hline Ma, J.; Zhang, T.; Dong, M. & $\begin{array}{l}\text { A novel ECG data compression method using } \\
\text { adaptive fourier decomposition with security } \\
\text { guarantee in e-health applications }\end{array}$ & IEEE J. Biomed. Health Inform. & 4.217 & 2015 & 52 & 112.00 \\
\hline $\begin{array}{l}\text { [61] Pedersen, S.J; Cooley, P.D.; } \\
\text { Mainsbridge, C. }\end{array}$ & $\begin{array}{l}\text { An e-health intervention designed to increase } \\
\text { workday energy expenditure by reducing } \\
\text { prolonged occupational sitting habits }\end{array}$ & $\begin{array}{l}\text { Work-A Journal of Prevention } \\
\text { Assessment \& Rehabilitation }\end{array}$ & 1.009 & 2014 & 62 & 112.00 \\
\hline $\begin{array}{l}\text { Quanbeck, A.; Gustafson, D.H.; } \\
\text { Marsch, L.A.; Chih, M.Y.; Kornfield, } \\
\text { R.; McTavish, F.; Johnson, R.; Brown, } \\
\text { R.T.; Mares, M.L.; Shah, D.V. }\end{array}$ & $\begin{array}{l}\text { Implementing a mobile health system to integrate } \\
\text { the treatment of addiction into primary care: } \mathrm{A} \\
\text { hybrid implementation-effectiveness study }\end{array}$ & J. Med. Internet Res. & 4.945 & 2018 & 21 & 111.00 \\
\hline Grünloh, C.; Cajander, A; Myreteg, G. & $\begin{array}{l}\text { The record is our work tool-physicians' framing of } \\
\text { a patient portal in Sweden }\end{array}$ & J. Med. Internet Res. & 4.945 & 2016 & 41 & 111.00 \\
\hline Sims, J. & $\begin{array}{l}\text { Communities of practice: telemedicine and online } \\
\text { medical communities }\end{array}$ & Technol. Forecast. Soc. Change & 3.815 & 2018 & 21 & 111.00 \\
\hline $\begin{array}{l}\text { [41] Litchman, M.; Rothwell, E.; } \\
\text { Edelman, L. }\end{array}$ & $\begin{array}{l}\text { The diabetes online community: older adults } \\
\text { supporting self-care through peer health }\end{array}$ & Patient Educ. Couns. & 2.821 & 2018 & 21 & 111.00 \\
\hline $\begin{array}{l}\text { [70] Paige, S.; Krieger, J.; Stellefson, } \\
\text { M.; Alber, J. }\end{array}$ & $\begin{array}{l}\text { eHealth literacy in chronic disease patients: An } \\
\text { item response theory analysis of the eHealth } \\
\text { literacy scale (eHEALS) }\end{array}$ & Patient Educ. Couns. & 2.821 & 2017 & 31 & 111.00 \\
\hline $\begin{array}{l}\text { Vijayakumar, V.; Priyan, M.K.; } \\
\text { Ushadevi, G.; Varatharajan, R.; } \\
\text { Manogaran, G.; Tarare, P.V. }\end{array}$ & $\begin{array}{l}\text { E-health cloud security using timing enabled } \\
\text { proxy re-encryption }\end{array}$ & Mobile Networks \& Applications & 2.390 & 2019 & 11 & 111.00 \\
\hline $\begin{array}{l}\text { Liddy, C.; Moroz, I.; Mihan, A.; } \\
\text { Nawar, N.; Keely, E. }\end{array}$ & $\begin{array}{l}\text { A systematic review of asynchronous, } \\
\text { provider-to-provider, electronic consultation } \\
\text { services to improve access to specialty care } \\
\text { available worldwide }\end{array}$ & Telemedicine and $\mathrm{e}$-Health & 1.996 & 2019 & 11 & 111.00 \\
\hline $\begin{array}{l}\text { Heisler, M.; Choi, H.; Palmisano, G.; } \\
\text { Mase, R.; Richardson, C.; Fagerlin, A.; } \\
\text { Montori, V.; Spencer, M.; An, L. }\end{array}$ & $\begin{array}{l}\text { Comparison of community health worker-led } \\
\text { diabetes medication decision-making support for } \\
\text { low-income Latino and African American adults } \\
\text { with diabetes using e-health tools versus } \\
\text { print materials }\end{array}$ & Annals of Internal Medicine & 19.315 & 2014 & 60 & 110.02 \\
\hline $\begin{array}{l}\text { Chouvarda, I.G.; Goulis, D.G.; } \\
\text { Lambrinoudaki, I.; Maglaveras, N. }\end{array}$ & $\begin{array}{l}\text { Connected health and integrated care: Toward new } \\
\text { models for chronic disease management }\end{array}$ & Maturitas & 3.654 & 2015 & 50 & 110.00 \\
\hline $\begin{array}{l}\text { Ferreri, F; Bourla, A.; Mouchabac, S.; } \\
\text { Karila, L. }\end{array}$ & $\begin{array}{l}\text { e-Addictology: An overview of new technologies } \\
\text { for assessing and intervening in } \\
\text { addictive behaviors }\end{array}$ & Front. Psychiatry & 3.162 & 2018 & 20 & 110.00 \\
\hline $\begin{array}{l}\text { Lee, E.W.; Denison, F.C.; Hor, K.; } \\
\text { Reynolds, R.M. }\end{array}$ & $\begin{array}{l}\text { Web-based interventions for prevention and } \\
\text { treatment of perinatal mood disorders: A } \\
\text { systematic review }\end{array}$ & $B M C$ Pregnancy and Childbirth & 2.413 & 2016 & 40 & 110.00 \\
\hline Pietro, C.D.; Francetic, I. & $\begin{array}{l}\text { E-health in Switzerland: the laborious adoption of } \\
\text { the federal law on electronic health records (EHR) } \\
\text { and health information exchange (HIE) networks }\end{array}$ & Health Policy & 2.075 & 2018 & 20 & 110.00 \\
\hline Bol, N.; Helberger, N.; Weert, J.C.M. & $\begin{array}{l}\text { Differences in mobile health app use: A source of } \\
\text { new digital inequalities? }\end{array}$ & Information Society & 1.860 & 2018 & 20 & 110.00 \\
\hline [99] Minoli, D.; Occhiogrosso, B. & Blockchain mechanisms for IoT security & Internet of Things & 0 & 2018 & 20 & 110.00 \\
\hline $\begin{array}{l}\text { Makhdoom, I.; Abolhasan, M.; } \\
\text { Lipman, J.; Liu, R.; Ni, W. }\end{array}$ & Anatomy of threats to the Internet of Things & $\begin{array}{l}\text { IEEE Communications Surveys and } \\
\text { Tutorials }\end{array}$ & 22.973 & 2019 & 9 & 109.02 \\
\hline Pussewalage, H.S.G.; Oleshchuk, V.A. & $\begin{array}{l}\text { Privacy preserving mechanisms for enforcing } \\
\text { security and privacy requirements in } \\
\text { e-health solutions }\end{array}$ & $\begin{array}{l}\text { International Journal of Information } \\
\text { Management }\end{array}$ & 5.063 & 2016 & 39 & 109.01 \\
\hline $\begin{array}{l}\text { Dimidjian, S.; Beck, A.; Felder, J.N.; } \\
\text { Boggs, J.M.; Gallop, R.; Segal, Z.V. }\end{array}$ & $\begin{array}{l}\text { Web-based mindfulness-based cognitive therapy } \\
\text { for reducing residual depressive symptoms: An } \\
\text { open trial and quasi-experimental comparison to } \\
\text { propensity score matched controls }\end{array}$ & Behaviour Research and Therapy & 4.309 & 2014 & 59 & 109.00 \\
\hline $\begin{array}{l}\text { Pedersen, N.; Thielsen, P.; Martinsen, } \\
\text { L.; Bennedsen, M.; Haaber, A.; } \\
\text { Langholz, E.; Végh, Z; Duricova, D.; } \\
\text { Jess, T.; Bell, S.; Burisch, J.; } \\
\text { Munkholm, P. }\end{array}$ & $\begin{array}{l}\text { EHealth: Individualization of mesalazine treatment } \\
\text { through a self-managed web-based solution in } \\
\text { mild-to-moderate ulcerative colitis }\end{array}$ & Inflammatory Bowel Diseases & 4.005 & 2014 & 59 & 109.00 \\
\hline $\begin{array}{l}\text { Aardoom, J.J.; Dingemans, A.E.; } \\
\text { Van Furth, E.F. }\end{array}$ & $\begin{array}{l}\text { E-health interventions for eating disorders: } \\
\text { Emerging findings, issues, and opportunities }\end{array}$ & Curr. Psychiatry Rep. & 3.816 & 2016 & 39 & 109.00 \\
\hline $\begin{array}{l}\text { Machado, G.; Pinheiro, M.; Lee, H.; } \\
\text { Ahmed, O.; Hendrick, P., Williams, C.; } \\
\text { Kamper, S. }\end{array}$ & $\begin{array}{l}\text { Smartphone apps for the self-management of low } \\
\text { back pain: A systematic review }\end{array}$ & $\begin{array}{l}\text { Best Practice \& Research Clinical } \\
\text { Rheumatology }\end{array}$ & 3.016 & 2016 & 39 & 109.00 \\
\hline $\begin{array}{l}\text { Slev, V.N.; Mistiaen, P.; Pasman, } \\
\text { H.R.W.; Verdonck-de Leeuw, I.M.; van } \\
\text { Uden-Kraan, C.F.; Francke, A.L. }\end{array}$ & $\begin{array}{l}\text { Effects of eHealth for patients and informal } \\
\text { caregivers confronted with cancer: A meta-review }\end{array}$ & $\begin{array}{l}\text { International Journal of Medical } \\
\text { Informatics }\end{array}$ & 2.721 & 2016 & 39 & 109.00 \\
\hline $\begin{array}{l}\text { Hossain, N.; Yokota, F.; Sultana, N.; } \\
\text { Ahmed, A. }\end{array}$ & $\begin{array}{l}\text { Factors influencing rural end-users' acceptance of } \\
\text { e-health in developing countries: A study on } \\
\text { portable health clinic in Bangladesh }\end{array}$ & Telemedicine and e-Health & 1.996 & 2019 & 9 & 109.00 \\
\hline $\begin{array}{l}\text { López-Jaquero, V; Montero, F; } \\
\text { Teruel, M. }\end{array}$ & $\begin{array}{l}\text { Influence awareness: Considering motivation in } \\
\text { computer-assisted rehabilitation }\end{array}$ & J. Ambient Intell. Humaniz. Comput. & 1.910 & 2019 & 9 & 109.00 \\
\hline $\begin{array}{l}\text { Robertson, N.; Polonsky, M.; } \\
\text { McQuilken, L. }\end{array}$ & $\begin{array}{l}\text { Are my symptoms serious Dr Google? A } \\
\text { resource-based typology of value co-destruction in } \\
\text { online self-diagnosis }\end{array}$ & $\begin{array}{c}\text { Australasian Marketing Journal } \\
\text { (AMJ) }\end{array}$ & 0 & 2014 & 59 & 109.00 \\
\hline $\begin{array}{l}\text { Li, F.; Li, Z.; Han, W.; Wu, T.; Chen, L.; } \\
\text { Guo, Y.; Chen, J. }\end{array}$ & $\begin{array}{l}\text { Cyberspace-oriented access control: A cyberspace } \\
\text { characteristics-based model and its policies }\end{array}$ & IEEE Internet Things J. & 9.516 & 2019 & 8 & 108.01 \\
\hline $\begin{array}{l}\text { Aghili, S.F.; Mala, H.; Shojafar, M.; } \\
\text { Peris-Lopez, P. }\end{array}$ & $\begin{array}{l}\text { LACO: Lightweight three-factor authentication, } \\
\text { access control and ownership transfer scheme for } \\
\text { e-health systems in IoT }\end{array}$ & Future Generation Computer Systems & 5.768 & 2019 & 8 & 108.01 \\
\hline
\end{tabular}


Table A1. Cont.

\begin{tabular}{|c|c|c|c|c|c|c|}
\hline Author & Title & Journal & $\begin{array}{l}\text { Impact } \\
\text { Factor }\end{array}$ & Year & Citations & InOrdinatio 10 \\
\hline $\begin{array}{l}\text { Yang, Y.; Zheng, X.; Liu, X.; Zhong, S.; } \\
\text { Chang, V. }\end{array}$ & $\begin{array}{l}\text { Cross-domain dynamic anonymous authenticated } \\
\text { group key management with symptom-matching } \\
\text { for e-health social system }\end{array}$ & Future Generation Computer Systems & 5.768 & 2018 & 18 & 108.01 \\
\hline Vitacca, M.; Montini, A.; Comini, L. & $\begin{array}{l}\text { How will telemedicine change clinical practice in } \\
\text { chronic obstructive pulmonary disease? }\end{array}$ & Ther. Adv. Respir. Dis. & 3.286 & 2018 & 18 & 108.00 \\
\hline $\begin{array}{l}\text { [71] Holmberg, C.; Berg, C.; Dahlgren, } \\
\text { J.; Lissner, L; Chaplin, J.E. }\end{array}$ & $\begin{array}{l}\text { Health literacy in a complex digital media } \\
\text { landscape: pediatric obesity patients' experiences } \\
\text { with online weight, food, and health information }\end{array}$ & Health Informatics $J$. & 2.297 & 2019 & 8 & 108.00 \\
\hline $\begin{array}{l}\text { Irving, M.; Stewart, R.; Spallek, H.; } \\
\text { Blinkhorn, A. }\end{array}$ & $\begin{array}{l}\text { Using teledentistry in clinical practice as an enabler } \\
\text { to improve access to clinical care: A qualitative } \\
\text { systematic review }\end{array}$ & J. Telemed. Telecare & 2.229 & 2018 & 18 & 108.00 \\
\hline $\begin{array}{l}\text { Saba, T.; Khan, S.U.; Islam, N.; Abbas, } \\
\text { N.; Rehman, A.; Javaid, N.; Anjum, A. }\end{array}$ & $\begin{array}{l}\text { Cloud-based decision support system for the } \\
\text { detection and classification of malignant cells in } \\
\text { breast cancer using breast cytology images }\end{array}$ & Microsc, Res. Tech. & 1.327 & 2019 & 8 & 108.00 \\
\hline $\begin{array}{l}\text { [8] De Grood, C.; Raissi, A.; Kwon, Y.; } \\
\text { Santana, M.J. }\end{array}$ & $\begin{array}{l}\text { Adoption of e-health technology by physicians: A } \\
\text { scoping review }\end{array}$ & J. Multidiscip. Healthc. & 0 & 2016 & 38 & 108.00 \\
\hline $\begin{array}{l}\text { Hamza, R.; Yan, Z.; Muhammad, K.; } \\
\text { Bellavista, P.; Titouna, F. }\end{array}$ & $\begin{array}{l}\text { A privacy-preserving cryptosystem for } \\
\text { IoT E-healthcare }\end{array}$ & Information Sciences & 5.524 & 2019 & 7 & 107.01 \\
\hline $\begin{array}{l}\text { Greenwood, D.; Blozis, S.; Young, H.; } \\
\text { Nesbitt, T.; Quinn, C. }\end{array}$ & $\begin{array}{l}\text { Overcoming clinical inertia: A randomized clinical } \\
\text { trial of a telehealth remote monitoring intervention } \\
\text { using paired glucose testing in adults with } \\
\text { type } 2 \text { diabetes }\end{array}$ & J. Med. Internet Res. & 4.945 & 2015 & 47 & 107.00 \\
\hline $\begin{array}{l}\text { Cozza, M.; Crevani, L.; Hallin, A.; } \\
\text { Schaeffer, J. }\end{array}$ & $\begin{array}{l}\text { Future ageing: Welfare technology practices for our } \\
\text { future older selves }\end{array}$ & Futures & 2.214 & 2019 & 7 & 107.00 \\
\hline Amato, F; Moscato, F. & $\begin{array}{l}\text { A model driven approach to data privacy } \\
\text { verification in e-health systems }\end{array}$ & Transactions on Data Privacy & 0 & 2015 & 47 & 107.00 \\
\hline $\begin{array}{l}\text { [10] Swinkels, I.C.S.; Huygens, M.W.J.; } \\
\text { Schoenmakers, T.M.; } \\
\text { Nijeweme-D'Hollosy, W.O.; Van } \\
\text { Velsen, L.; Vermeulen, J.; } \\
\text { Schoone-Harmsen, M.; Jansen, } \\
\text { Y.J.F.M.; Van Schayck, OC.C.; Friele, } \\
\text { R.; Witte, L. de }\end{array}$ & $\begin{array}{l}\text { Lessons learned from a living lab on the broad } \\
\text { adoption of eHealth in primary health care }\end{array}$ & J. Med. Internet Res. & 4.945 & 2018 & 16 & 106.00 \\
\hline $\begin{array}{l}\text { Volker, D.; Zijlstra-Vlasveld, M.C.; } \\
\text { Anema, J.R.; Beekman, A.T.F.; } \\
\text { Brouwers, E.P.M.; Emons, W.H.M.; } \\
\text { Van Lomwel, A.G.C.; Van Der } \\
\text { Feltz-Cornelis, C.M. }\end{array}$ & $\begin{array}{l}\text { Effectiveness of a blended web-based intervention } \\
\text { on return to work for sick-listed employees with } \\
\text { common mental disorders: Results of a cluster } \\
\text { randomized controlled trial }\end{array}$ & J. Med. Internet Res. & 4.945 & 2015 & 46 & 106.00 \\
\hline $\begin{array}{l}\text { Børøsund, E.; Cvancarova, M.; Moore, } \\
\text { S.; Ekstedt, M.; Ruland, C. }\end{array}$ & $\begin{array}{l}\text { Comparing effects in regular practice of } \\
\text { e-communication and web-based self-management } \\
\text { support among breast cancer patients: Preliminary } \\
\text { results from a randomized controlled trial }\end{array}$ & J. Med. Internet Res. & 4.945 & 2014 & 56 & 106.00 \\
\hline $\begin{array}{l}\text { Noonan, V.K.; Lyddiatt, A.; Ware, P.; } \\
\text { Jaglal, S.B.; Riopelle, R.J;; Bingham III, } \\
\text { C.O.; Figueiredo, S.; Sawatzky, R.; } \\
\text { Santana, M.; Bartlett, S.J.;Ahmed, S. }\end{array}$ & $\begin{array}{l}\text { Montreal Accord on Patient-Reported Outcomes } \\
\text { (PROs) use series-Paper 3: Patient-reported } \\
\text { outcomes can facilitate shared decision-making } \\
\text { and guide self-management }\end{array}$ & J. Clin. Epidemiol. & 4.65 & 2017 & 26 & 106.00 \\
\hline $\begin{array}{l}\text { Nguyen, D.C.; Pathirana, P.N.; Ding, } \\
\text { M.; Seneviratne, A. }\end{array}$ & $\begin{array}{l}\text { Blockchain for secure EHRs sharing of mobile } \\
\text { cloud based e-health system }\end{array}$ & Access, IEEE & 4.098 & 2019 & 6 & 106.00 \\
\hline [97] Wang, P.; Ye, F; Chen, X.; Qian, Y. & $\begin{array}{l}\text { Datanet: Deep learning based encrypted network } \\
\text { traffic classification in SDN Home Gateway }\end{array}$ & Access, IEEE & 4.098 & 2018 & 16 & 106.00 \\
\hline $\begin{array}{l}\text { Steins, D.; Dawes, H; Esser, P.; } \\
\text { Collett, J. }\end{array}$ & $\begin{array}{l}\text { Wearable accelerometry-based technology capable } \\
\text { of assessing functional activities in neurological } \\
\text { populations in community settings: A } \\
\text { systematic review }\end{array}$ & J. Neuroeng. Rehabil. & 3.582 & 2014 & 56 & 106.00 \\
\hline [54] Wass, S.; Vimarlund, V.; Ros, A. & $\begin{array}{l}\text { Exploring patients' perceptions of accessing } \\
\text { electronic health records: Innovation in healthcare }\end{array}$ & Health Informatics J. & 2.297 & 2019 & 6 & 106.00 \\
\hline $\begin{array}{l}\text { Hall, A.K.; Bernhardt, J.M.; Dodd, V.; } \\
\text { Vollrath, M.W. }\end{array}$ & $\begin{array}{l}\text { The digital health divide: Evaluating online health } \\
\text { information access and use among older adults }\end{array}$ & Health Education \& Behavior & 2.190 & 2015 & 46 & 106.00 \\
\hline $\begin{array}{l}\text { Girault, A.; Ferrua, M.; Lalloué, B.; } \\
\text { Sicotte, C.; Fourcade, A.; Yatim, F.; } \\
\text { Hébert, G.; Palma, M.D.; Minvielle, E. }\end{array}$ & $\begin{array}{l}\text { Internet-based technologies to improve cancer care } \\
\text { coordination: Current use and attitudes among } \\
\text { cancer patients }\end{array}$ & Eur. J. Cancer & 6.68 & 2015 & 45 & 105.01 \\
\hline $\begin{array}{l}\text { Khan, S.U.; Islam, N.; Jan, Z.; Din, I.U.; } \\
\text { Khan, A.; Faheem, Y. }\end{array}$ & $\begin{array}{l}\text { An e-health care services framework for the } \\
\text { detection and classification of breast cancer in } \\
\text { breast cytology images as an IoMT application }\end{array}$ & Future Generation Computer Systems & 5.768 & 2019 & 5 & 105.01 \\
\hline Hsu, W.; Chiang, C.; Yang, S. & $\begin{array}{l}\text { The effect of individual factors on health behaviors } \\
\text { among college students: The mediating effects of } \\
\text { eHealth literacy }\end{array}$ & J. Med. Internet Res. & 4.945 & 2014 & 55 & 105.00 \\
\hline [18] Parikh, S.V:; Huniewicz, P. & $\begin{array}{l}\text { E-health: An overview of the uses of the internet, } \\
\text { social media, apps, and websites for mood } \\
\text { disorders }\end{array}$ & Curr. Opin. Psychiatry & 4.483 & 2015 & 45 & 105.00 \\
\hline $\begin{array}{l}\text { Wazid, M.; Das, A.K.; Kumar, N.; } \\
\text { Odelu, V.; Reddy, A.G.; Parks, K.; } \\
\text { Parks, Y. }\end{array}$ & $\begin{array}{l}\text { Design of lightweight authentication and key } \\
\text { agreement protocol for vehicular ad hoc networks }\end{array}$ & Access, IEEE & 4.098 & 2017 & 25 & 105.00 \\
\hline $\begin{array}{l}\text { Tosi, J.; Taffoni, F.; Santacatterina, M.; } \\
\text { Sannino, R.; Formica, D. }\end{array}$ & $\begin{array}{l}\text { Performance evaluation of bluetooth low energy: A } \\
\text { systematic review }\end{array}$ & Sensors & 3.031 & 2017 & 25 & 105.00 \\
\hline $\begin{array}{l}\text { Wagenaar, K.P.; Broekhuizen, B.D.L.; } \\
\text { Jaarsma, T.; Kok, I.; Mosterd, A.; } \\
\text { Willems, F.F.; Linssen, G.C.M.; Agema, } \\
\text { W.R.P.; Anneveldt, S.; Lucas, } \\
\text { C.M.H.H.; Mannaerts, H.F..; Wajon, } \\
\text { E.M.C.J.; Dickstein, K.; Cramer, M.J.; } \\
\text { Landman, M.A.J.; Hoes, A.W.; } \\
\text { Rutten, F.H. }\end{array}$ & $\begin{array}{l}\text { Effectiveness of the European Society of } \\
\text { Cardiology/Heart Failure Association website } \\
\text { "heartfailurematters.org" and an e-health adjusted } \\
\text { care pathway in patients with stable heart failure: } \\
\text { Results of the "e-Vita HF" randomized } \\
\text { controlled trial }\end{array}$ & Eur. J. Heart Fail. & 2.784 & 2019 & 5 & 105.00 \\
\hline Wernhart, A.; Gahbauer, S.; Haluza, D. & $\begin{array}{l}\text { eHealth and telemedicine: Practices and beliefs } \\
\text { among healthcare professionals and medical } \\
\text { students at a medical university }\end{array}$ & Plos One & 2.776 & 2019 & 5 & 105.00 \\
\hline
\end{tabular}


Table A1. Cont.

\begin{tabular}{|c|c|c|c|c|c|c|}
\hline Author & Title & Journal & $\begin{array}{l}\text { Impact } \\
\text { Factor }\end{array}$ & Year & Citations & InOrdinatio 10 \\
\hline $\begin{array}{l}\text { Maramba, I.; Chatterjee, A.; Newman, } \\
\text { C. }\end{array}$ & $\begin{array}{l}\text { Methods of usability testing in the development of } \\
\text { eHealth applications: A scoping review }\end{array}$ & $\begin{array}{l}\text { International Journal of Medical } \\
\text { Informatics }\end{array}$ & 2.721 & 2019 & 5 & 105.00 \\
\hline $\begin{array}{c}\text { Daher, J.; Vijh, R.; Linthwaite, B.; } \\
\text { Dave, S.; Kim, J.; Dheda, K.; Peter, T.; } \\
\text { Pai, N. }\end{array}$ & $\begin{array}{l}\text { Do digital innovations for HIV and sexually } \\
\text { transmitted infections work? Results from a } \\
\text { systematic review (1996-2017) }\end{array}$ & BMJ Open & 2.376 & 2017 & 25 & 105.00 \\
\hline Bucci, S.; Schwannauer, M.; Berry, N. & $\begin{array}{l}\text { The digital revolution and its impact on mental } \\
\text { health care }\end{array}$ & Psychol Psychother & 2.244 & 2019 & 5 & 105.00 \\
\hline Oueida, S.; Aloqaily, M.; Ionescu, S. & $\begin{array}{l}\text { A smart healthcare reward model for resource } \\
\text { allocation in smart city }\end{array}$ & Multimedia Tools and Applications & 2.101 & 2019 & 5 & 105.00 \\
\hline Zhao, X.; Yang, B.; Wong, C.-W. & $\begin{array}{l}\text { Analyzing trend for U.S. Immigrants' e-health } \\
\text { engagement from } 2008 \text { to } 2013\end{array}$ & Health Communication & 1.846 & 2019 & 5 & 105.00 \\
\hline $\begin{array}{l}\text { Keasberry, J.; Scott, I.A.; Sullivan, C.; } \\
\text { Staib, A.; Ashby, R. }\end{array}$ & $\begin{array}{l}\text { Going digital: A narrative overview of the clinical } \\
\text { and organisational impacts of eHealth technologies } \\
\text { in hospital practice }\end{array}$ & Australian Health Review & 1.228 & 2017 & 25 & 105.00 \\
\hline $\begin{array}{l}\text { Helle, C.; Hillesund, E.; Wills, A.; } \\
\text { Øverby, N. }\end{array}$ & $\begin{array}{l}\text { Evaluation of an eHealth intervention aiming to } \\
\text { promote healthy food habits from infancy-the } \\
\text { Norwegian randomized controlled trial Early Food } \\
\text { for Future Health }\end{array}$ & IJBNPA & 6.037 & 2019 & 4 & 104.01 \\
\hline $\begin{array}{l}\text { Hammersley, M.; Okely, A.; } \\
\text { Batterham, M.; Jones, R. }\end{array}$ & $\begin{array}{l}\text { An internet-based childhood obesity prevention } \\
\text { program (TIMe2bhealthy) for parents of } \\
\text { preschool-aged children: randomized } \\
\text { controlled trial }\end{array}$ & J. Med. Internet Res. & 4.945 & 2019 & 4 & 104.00 \\
\hline $\begin{array}{l}\text { Adam, M.; McMahon, S.; Prober, C.; } \\
\text { Bärnighausen, T. }\end{array}$ & $\begin{array}{l}\text { Human-centered design of video-based health } \\
\text { education: An iterative, collaborative, } \\
\text { community-based approach }\end{array}$ & J. Med. Internet Res. & 4.945 & 2019 & 4 & 104.00 \\
\hline $\begin{array}{l}\text { MacDonald, G.; Townsend, A.; Adam, } \\
\text { P.; Li, L.; Kerr, S.; McDonald, M.; } \\
\text { Backman, C. }\end{array}$ & $\begin{array}{l}\text { eHealth technologies, multimorbidity, and the } \\
\text { office visit: Qualitative interview study on the } \\
\text { perspectives of physicians and nurses }\end{array}$ & J. Med. Internet Res. & 4.945 & 2018 & 14 & 104.00 \\
\hline $\begin{array}{l}\text { Orchard, J.; Neubeck, L.; Freedman, B.; } \\
\text { Li, J.; Webster, R.; Zwar, N.; Gallagher, } \\
\text { R.; Ferguson, C.; Lowres, N. }\end{array}$ & $\begin{array}{l}\text { eHealth tools to provide structured assistance for } \\
\text { atrial fibrillation screening, management, and } \\
\text { guideline-recommended therapy in metropolitan } \\
\text { general practice: The AF-SMART study }\end{array}$ & J. Am. Heart Assoc. & 4.660 & 2019 & 4 & 104.00 \\
\hline $\begin{array}{l}\text { Alwashmi, M.F; Hawboldt, J.; Davis, } \\
\text { E.; Fetters, M.D. }\end{array}$ & $\begin{array}{l}\text { The iterative convergent design for mobile health } \\
\text { usability testing: Mixed-methods approach }\end{array}$ & JMIR $m$ Health and $u$ Health & 4.301 & 2019 & 4 & 104.00 \\
\hline $\begin{array}{c}\text { Goetz, M.; Muller, M.; Matthies, L.M.; } \\
\text { Hansen, J.; Doster, A.; Szabo, A.; } \\
\text { Pauluschke-Froehlich, J.; Abele, H., } \\
\text { Sohn, C.; Wallwiener, M.; Wallwiener, } \\
\text { S. }\end{array}$ & $\begin{array}{l}\text { Perceptions of patient engagement applications } \\
\text { during pregnancy: A qualitative assessment of the } \\
\text { patient's perspective }\end{array}$ & JMIR $m$ Health and $u$ Health & 4.301 & 2017 & 24 & 104.00 \\
\hline $\begin{array}{l}\text { Warrington, L.; Absolom, K.; Velikova, } \\
\text { G. }\end{array}$ & $\begin{array}{l}\text { Integrated care pathways for cancer survivors-a } \\
\text { role for patient-reported outcome measures and } \\
\text { health informatics }\end{array}$ & Acta Oncologica & 3.298 & 2015 & 44 & 104.00 \\
\hline $\begin{array}{c}\text { Gossec, L.; Molto, A.; Romand, X.; } \\
\text { Puyraimond-Zemmour, D.; Lavielle, } \\
\text { M.; Beauvais, C.; Senbel, E.; Flipo, } \\
\text { R.M.; Pouplin, S.; Richez, C.; Saraux, } \\
\text { A.; Mézières, M.; Gutermann, L., } \\
\text { Gaudin, P.; Wendling, D.; } \\
\text { Dougados, M. }\end{array}$ & $\begin{array}{l}\text { Recommendations for the assessment and } \\
\text { optimization of adherence to disease-modifying } \\
\text { drugs in chronic inflammatory rheumatic diseases: } \\
\text { A process based on literature reviews and } \\
\text { expert consensus }\end{array}$ & Joint Bone Spine & 3.278 & 2019 & 4 & 104.00 \\
\hline $\begin{array}{l}\text { Kitsios, F.; Stefanakakis, S.; } \\
\text { Kamariotou, M.; Dermentzoglou, L. }\end{array}$ & $\begin{array}{c}\text { E-service evaluation: User satisfaction } \\
\text { measurement and implications in health sector }\end{array}$ & Computer Standards \& Interfaces & 2.441 & 2019 & 4 & 104.00 \\
\hline $\begin{array}{l}\text { Lindner, P; Miloff, A.; Zetterlund, E.; } \\
\text { Reuterskiold, L; Andersson, G.; } \\
\text { Carlbring, P. }\end{array}$ & $\begin{array}{l}\text { Attitudes toward and familiarity with virtual } \\
\text { reality therapy among practicing cognitive } \\
\text { behavior therapists: A cross-sectional survey study } \\
\text { in the era of consumer VR platforms }\end{array}$ & Frontiers in Psychology & 2.129 & 2019 & 4 & 104.00 \\
\hline Cho, Y.M.; Lee, S.; Islam, S.; Kim, S.Y. & $\begin{array}{l}\text { Theories applied to m-health interventions for } \\
\text { behavior change in low- and middle-income } \\
\text { countries: A systematic review }\end{array}$ & Telemedicine and $\mathrm{e}$-Health & 1.996 & 2018 & 14 & 104.00 \\
\hline Terrasse, M., Gorin, M.; Sisti, D. & Social media, e-health, and medical ethics & Hastings Center Report & 1.728 & 2019 & 4 & 104.00 \\
\hline $\begin{array}{l}\text { Öberg, U.; Orre, C.; Isaksson, U.; } \\
\text { Schimmer, R.; Larsson, H.; } \\
\text { Hörnsten, A. }\end{array}$ & $\begin{array}{l}\text { Swedish primary healthcare nurses' perceptions of } \\
\text { using digital eHealth services in support of } \\
\text { patient self-management }\end{array}$ & Scand. J. Caring Sci. & 1.642 & 2018 & 14 & 104.00 \\
\hline Razmak, J.; Belanger, C. & $\begin{array}{l}\text { Using the technology acceptance model to predict } \\
\text { patient attitude toward personal health records in } \\
\text { regional communities }\end{array}$ & Information Technology \& People & 1.263 & 2018 & 14 & 104.00 \\
\hline $\begin{array}{l}\text { [96] Thomas, N.; Bless, J.J.; } \\
\text { Alderson-Day, B.; Bell, I.H.; Cella, M.; } \\
\text { Craig, T; Delespaul, P.; Hugdahl, K.; } \\
\text { Laloyaux, J.; Larvi, F.; Lincoln, T.M.; } \\
\text { Schlier, B.; Urwwler, P.; } \\
\text { D.; Jan den Berg, } \\
\text { D.; Jardri, R. R. }\end{array}$ & $\begin{array}{l}\text { Potential applications of digital technology in } \\
\text { assessment, treatment, and self-help } \\
\text { for hallucinations }\end{array}$ & Schizophr. Bull. & 7.289 & 2019 & 3 & 103.01 \\
\hline $\begin{array}{l}\text { Willmott, T.; Pang, B.; Rundle-Thiele, } \\
\text { S.; Badejo, A. }\end{array}$ & $\begin{array}{l}\text { Weight management in young adults: Systematic } \\
\text { review of electronic health intervention } \\
\text { components and outcomes }\end{array}$ & J. Med. Internet Res. & 4.945 & 2019 & 3 & 103.00 \\
\hline $\begin{array}{l}\text { Van Den Heuvel, J.; Groenhof, T.; } \\
\text { Veerbeek, J.; Van Solinge, W.; Lely, A.; } \\
\text { Franx, A.; Bekker, M. }\end{array}$ & $\begin{array}{l}\text { eHealth as the next-generation perinatal care: An } \\
\text { overview of the literature }\end{array}$ & J. Med. Internet Res. & 4.945 & 2018 & 13 & 103.00 \\
\hline $\begin{array}{l}\text { Chenthara, S.; Ahmed, K.; Wang, H.; } \\
\text { Whittaker, F. }\end{array}$ & $\begin{array}{l}\text { Security and privacy-preserving challenges of } \\
\text { e-health solutions in cloud computing }\end{array}$ & Access, IEEE & 4.098 & 2019 & 3 & 103.00 \\
\hline $\begin{array}{l}\text { [94] Vloothuis, J.D.M.; Mulder, M.; } \\
\text { Nijland, R.H.M.; Goedhart, Q.S.; } \\
\text { Konijnenbelt, M.; Mulder, H.; } \\
\text { Hertogh, C.M.P.M.; van Tulder, M.; } \\
\text { van Wegen, E.E.H.; Kwakkel, G. }\end{array}$ & $\begin{array}{l}\text { Caregiver-mediated exercises with e-health } \\
\text { support for early supported discharge after stroke } \\
\text { (CARE4STROKE): A randomized controlled trial }\end{array}$ & Plos One & 2.776 & 2019 & 3 & 103.00 \\
\hline Cheatle, M. & $\begin{array}{l}\text { Biopsychosocial approach to assessing and } \\
\text { managing patients with chronic pain }\end{array}$ & Medical Clinics of North America & 2.716 & 2016 & 33 & 103.00 \\
\hline
\end{tabular}


Table A1. Cont.

\begin{tabular}{|c|c|c|c|c|c|c|}
\hline Author & Title & Journal & $\begin{array}{l}\text { Impact } \\
\text { Factor }\end{array}$ & Year & Citations & InOrdinatio 10 \\
\hline Ahmed, B.; Dannhauser, T.; Philip, N. & $\begin{array}{l}\text { A systematic review of reviews to identify key } \\
\text { research opportunities within the field of } \\
\text { eHealth implementation }\end{array}$ & J. Telemed. Telecare & 2.229 & 2019 & 3 & 103.00 \\
\hline $\begin{array}{l}\text { [48] Schettini, P.; Shah, K.; O'Leary, C.; } \\
\text { Patel, M.; Anderson, J.; Cho, A.; Long, } \\
\text { A.; Bosworth, H.; Cameron, C. }\end{array}$ & $\begin{array}{l}\text { Keeping care connected: E-Consultation program } \\
\text { improves access to nephrology care }\end{array}$ & J. Telemed. Telecare & 2.229 & 2019 & 3 & 103.00 \\
\hline $\begin{array}{l}\text { Karekla, M.; Kasinopoulos, O.; Neto, } \\
\text { D.; Ebert, D.; Van Daele, T.; } \\
\text { Nordgreen, T.; Höfer, S.; Oeverland, } \\
\text { S.; Jensen, K. }\end{array}$ & $\begin{array}{l}\text { Best practices and recommendations for digital } \\
\text { interventions to improve engagement and } \\
\text { adherence in chronic illness sufferers }\end{array}$ & European Psychologist & 2.167 & 2019 & 3 & 103.00 \\
\hline De Rosis, S.; Barsanti, S. & $\begin{array}{l}\text { Patient satisfaction, e-health and the evolution of } \\
\text { the patient-general practitioner relationship: } \\
\text { Evidence from an Italian survey }\end{array}$ & Health Policy & 2.075 & 2016 & 33 & 103.00 \\
\hline $\begin{array}{l}\text { Zonneveld, M.; Patomella, A.H.; } \\
\text { Asaba, E.; Guidetti, S. }\end{array}$ & $\begin{array}{l}\text { The use of information and communication } \\
\text { technology in healthcare to improve participation } \\
\text { in everyday life: A scoping review }\end{array}$ & Disability and rehabilitation & 2.054 & 2019 & 3 & 103.00 \\
\hline Leung, L.; Chen, C. & $\begin{array}{l}\text { E-health/m-health adoption and lifestyle } \\
\text { improvements: Exploring the roles of technology } \\
\text { readiness, the expectation-confirmation model, and } \\
\text { health-related information activities }\end{array}$ & Telecomm Policy & 2.000 & 2019 & 3 & 103.00 \\
\hline Kerst, A.; Zielasek, Jü.; Gaebel, W. & $\begin{array}{l}\text { Smartphone applications for depression: A } \\
\text { systematic literature review and a survey of health } \\
\text { care professionals\&039; attitudes towards their use } \\
\text { in clinical practice }\end{array}$ & Eur. Arch. Psychiatr. Clin. Neurosci. & 0 & 2019 & 3 & 103.00 \\
\hline $\begin{array}{l}\text { Kubendiran, M.; Singh, S.; } \\
\text { Sangaiah, A.K. }\end{array}$ & $\begin{array}{l}\text { Enhanced security framework for e-health systems } \\
\text { using blockchain }\end{array}$ & $\begin{array}{l}\text { Journal of Information Processing } \\
\text { Systems }\end{array}$ & 0 & 2019 & 3 & 103.00 \\
\hline $\begin{array}{l}\text { Celesti, A.; Fazio, M.; Márquez, F.; } \\
\text { Glikson, A.; Mauwa, H.; Bagula, A., } \\
\text { Celesti, F.; Villari, M. }\end{array}$ & $\begin{array}{l}\text { How to develop IoT cloud e-health systems based } \\
\text { on fiware: A lesson learnt }\end{array}$ & $\begin{array}{l}\text { Journal of Sensor and Actuator } \\
\text { Networks }\end{array}$ & 0 & 2019 & 3 & 103.00 \\
\hline Alvarez, C.; Fedock, G. & $\begin{array}{l}\text { Addressing intimate partner violence with Latina } \\
\text { women: A call for research }\end{array}$ & Trauma Violence Abuse & 0 & 2018 & 13 & 103.00 \\
\hline $\begin{array}{l}\text { Van Der Meij, E.; Anema, J.R.; } \\
\text { Leclercq, W.K.G.; Bongers, M.Y.; } \\
\text { Consten, E.C.J.; Koops, S.E.S.; Van De } \\
\text { Ven, P.M.; Terwee, C.B.; Van Dongen, } \\
\text { J.M.; Schaafsma, F.G.; Meijerink, } \\
\text { W.J.H.J.; Bonjer, H.J.; Huirne, J.A.F. }\end{array}$ & $\begin{array}{l}\text { Personalised perioperative care by e-health after } \\
\text { intermediate-grade abdominal surgery: A } \\
\text { multicentre, single-blind, randomised, } \\
\text { placebo-controlled trial }\end{array}$ & Lancet & 59.102 & 2018 & 12 & 102.06 \\
\hline $\begin{array}{l}\text { Islam, N.; Faheem, Y.; Din, I.U.; Talha, } \\
\text { M.; Guizani, M.; Khalil, M. }\end{array}$ & $\begin{array}{l}\text { A blockchain-based fog computing framework for } \\
\text { activity recognition as an application to } \\
\text { e-healthcare services }\end{array}$ & Future Generation Computer Systems & 5.768 & 2019 & 2 & 102.01 \\
\hline $\begin{array}{l}\text { Peddi, V.B.; Kuhad, P.; Yassine, A.; } \\
\text { Pouladzadeh, P.; Shirmohammadi, S.; } \\
\text { Shirehjini, A.A.N. }\end{array}$ & $\begin{array}{l}\text { An intelligent cloud-based data processing broker } \\
\text { for mobile e-health multimedia applications }\end{array}$ & Future Generation Computer Systems & 5.768 & 2017 & 22 & 102.01 \\
\hline $\begin{array}{l}\text { Chen, Y.; Crespi, N.; Ortiz, A.M.; } \\
\text { Shu, L. }\end{array}$ & $\begin{array}{l}\text { Reality mining: A prediction algorithm for disease } \\
\text { dynamics based on mobile Big Data }\end{array}$ & Information Sciences & 5.524 & 2017 & 22 & 102.01 \\
\hline $\begin{array}{l}\text { Shaw, T.; Janssen, A.; Crampton, R.; } \\
\text { O'Leary, F.; Hoyle, P.; Jones, A.; Shetty, } \\
\text { A.; Gunja, N.; Ritchie, A.; Spallek, H.; } \\
\text { Solman, A.; Kay, J., Makeham, M.; } \\
\text { Harnett, P. }\end{array}$ & $\begin{array}{l}\text { Attitudes of health professionals to using routinely } \\
\text { collected clinical data for performance feedback } \\
\text { and personalised professional development }\end{array}$ & Med. J. Aust. & 5.332 & 2019 & 2 & 102.01 \\
\hline $\begin{array}{l}\text { [89] Sakakibara, B.; Chakrabarti, S.; } \\
\text { Krahn, A.; Mackay, M.; Sedlak, T.; } \\
\text { Singer, J.; Whitehurst, D.; Lear, S. }\end{array}$ & $\begin{array}{l}\text { Delivery of peer support through a } \\
\text { self-management mHealth intervention (healing } \\
\text { circles) in patients with cardiovascular disease: } \\
\text { Protocol for a randomized controlled trial }\end{array}$ & J. Med. Internet Res. & 4.945 & 2019 & 2 & 102.00 \\
\hline $\begin{array}{l}\text { Vis, C.; Ruwaard, J.; Finch, T.; Rapley, } \\
\text { T;; De Beurs, D.; Van Stel, H.; Van } \\
\text { Lettow, B; Mol, M.; Kleiboer, A.; } \\
\text { Riper, H.; Smit, J. }\end{array}$ & $\begin{array}{l}\text { Toward an objective assessment of implementation } \\
\text { processes for innovations in health care: } \\
\text { Psychometric evaluation of the normalization } \\
\text { Measure Development (NOMAD) questionnaire } \\
\text { among mental health care professionals }\end{array}$ & J. Med. Internet Res. & 4.945 & 2019 & 2 & 102.00 \\
\hline $\mathrm{Wu}, \mathrm{B}$. & $\begin{array}{l}\text { Patient continued use of online health care } \\
\text { communities: Web mining of } \\
\text { patient-doctor communication }\end{array}$ & J. Med. Internet Res. & 4.945 & 2018 & 12 & 102.00 \\
\hline $\begin{array}{l}\text { Hossain, M.; Poon, C.; Dong, Y.; Lo, L.; } \\
\text { Cheng, J. }\end{array}$ & $\begin{array}{l}\text { Development of social sustainability assessment } \\
\text { method and a comparative case study on assessing } \\
\text { recycled construction materials }\end{array}$ & $\begin{array}{l}\text { International Journal of Life Cycle } \\
\text { Assessment }\end{array}$ & 4.868 & 2018 & 12 & 102.00 \\
\hline $\begin{array}{l}\text { Barteit, S.; Neuhann, F.; Barnighausen, } \\
\text { T.; Luders, S.; Malunga, G.; Chileshe, } \\
\text { G.; Marimo, C.; Jahn, A. }\end{array}$ & $\begin{array}{l}\text { Perspectives of nonphysician clinical students and } \\
\text { medical lecturers on tablet-based health care } \\
\text { practice support for medical education in Zambia, } \\
\text { Africa: Qualitative study }\end{array}$ & JMIR $m$ Health and $u$ Health & 4.301 & 2019 & 2 & 102.00 \\
\hline Antonio, M.; Petrovskaya, O.; Lau, F. & $\begin{array}{l}\text { Is research on patient portals attuned to health } \\
\text { equity? A scoping review }\end{array}$ & J. Am. Med. Inform. Assoc. & 4.292 & 2019 & 2 & 102.00 \\
\hline $\begin{array}{l}\text { Tursunbayeva, A.; Bunduchi, R.; } \\
\text { Franco, M.; Pagliari, C. }\end{array}$ & $\begin{array}{l}\text { Human resource information systems in health } \\
\text { care: A systematic evidence review }\end{array}$ & J. Am. Med. Inform. Assoc. & 4.292 & 2017 & 22 & 102.00 \\
\hline $\begin{array}{l}\text { Odelu, V.; Saha, S.; Prasath, R.; } \\
\text { Sadineni, L.; Conti, M.; Jo, M. }\end{array}$ & $\begin{array}{l}\text { Efficient privacy preserving device authentication } \\
\text { in WBANs for industrial e-health applications }\end{array}$ & Computers \& Security & 3.062 & 2019 & 2 & 102.00 \\
\hline $\begin{array}{l}\text { Devan, H.; Perry, M.A.; Van Hattem, } \\
\text { A.; Thurlow, G.; Shepherd, S., } \\
\text { Muchemwa, C.; Grainger, R. }\end{array}$ & $\begin{array}{l}\text { Do pain management websites foster } \\
\text { self-management support for people with } \\
\text { persistent pain? A scoping review }\end{array}$ & Patient Educ. Couns. & 2.821 & 2019 & 2 & 102.00 \\
\hline $\begin{array}{l}\text { Carcone, A.; Hasan, M.; Alexander, G.; } \\
\text { Dong, M.; Eggly, S.; Hartlieb, K.; Naar, } \\
\text { S.; MacDonell, K.; Kotov, A. }\end{array}$ & $\begin{array}{l}\text { Developing machine learning models for } \\
\text { behavioral coding }\end{array}$ & J. Pediatr. Psychol. & 2.670 & 2019 & 2 & 102.00 \\
\hline $\begin{array}{l}\text { Lo Presti, L.; Testa, M.; Marino, V.; } \\
\text { Singer, P. }\end{array}$ & $\begin{array}{l}\text { Engagement in healthcare systems: Adopting } \\
\text { digital tools for a sustainable approach }\end{array}$ & Sustainability & 2.592 & 2019 & 2 & 102.00 \\
\hline $\begin{array}{l}\text { Ross, J.; Stevenson, F.; Lau, R.; } \\
\text { Murray, E. }\end{array}$ & $\begin{array}{l}\text { Exploring the challenges of implementing e-health: } \\
\text { A protocol for an update of a systematic review } \\
\text { of reviews }\end{array}$ & BMJ Open & 2.376 & 2015 & 42 & 102.00 \\
\hline
\end{tabular}


Table A1. Cont.

\begin{tabular}{|c|c|c|c|c|c|c|}
\hline Author & Title & Journal & $\begin{array}{l}\text { Impact } \\
\text { Factor }\end{array}$ & Year & Citations & InOrdinatio 10 \\
\hline $\begin{array}{l}\text { Heathcote, L.; Pate, J.; Park, A.; Leake, } \\
\text { H.; Lorimer Moseley, G.; Kronman, C.; } \\
\text { Fischer, M.; Timmers, I.; Simons, L. }\end{array}$ & Pain neuroscience education on YouTube & PEERJ & 2.353 & 2019 & 2 & 102.00 \\
\hline Azeez, N.A.; Der Vyver, C. & $\begin{array}{l}\text { Security and privacy issues in e-health cloud-based } \\
\text { system: A comprehensive content analysis }\end{array}$ & Egyptian Informatics Journal & 2.306 & 2019 & 2 & 102.00 \\
\hline $\begin{array}{l}\text { Prado, G.; Estrada, Y;; Rojas, L.M.; } \\
\text { Bahamon, M.; Pantin, H.; Nagarsheth, } \\
\text { M.; Gwynn, L.; Ofir, A.Y.; Forster, } \\
\text { L.Q., Torres, N.; Brown, C.H. }\end{array}$ & $\begin{array}{l}\text { Rationale and design for eHealth Familias Unidas } \\
\text { Primary Care: A drug use, sexual risk behavior, } \\
\text { and STI preventive intervention for Hispanic youth } \\
\text { in pediatric primary care clinics }\end{array}$ & Contemp. Clin. Trials & 2.280 & 2019 & 2 & 102.00 \\
\hline $\begin{array}{l}\text { Guertler, D.; Moehring, A.; Krause, K.; } \\
\text { Eck, S.; Batra, A.; Chenot, J.-F.; } \\
\text { Freyer-Adam, J.; Ulbricht, S.; Rumpf, } \\
\text { H.-J; Bischof, G.; John, U.; Meyer, C. }\end{array}$ & $\begin{array}{l}\text { Proactive multipurpose health risk screening in } \\
\text { health care settings: Methods, design, and reach }\end{array}$ & Int. J. Method Psych. & 2.276 & 2019 & 2 & 102.00 \\
\hline $\begin{array}{l}\text { Possemato, K.; Johnson, E.M.; Emery, } \\
\text { J.B.; Wade, M.; Acosta, M.C.; Marsch, } \\
\text { L.A.; Rosenblum, A.; Maisto, S.A. }\end{array}$ & $\begin{array}{l}\text { A pilot study comparing peer supported } \\
\text { web-based CBT to self-managed web CBT for } \\
\text { primary care veterans with PTSD and hazardous } \\
\text { alcohol use }\end{array}$ & Psychiatric Rehabilitation Journal & 2.270 & 2019 & 2 & 102.00 \\
\hline $\begin{array}{l}\text { Hossain, N.; Sampa, M.B.; Yokota, F; } \\
\text { Fukuda, A.; Ahmed, A. }\end{array}$ & $\begin{array}{l}\text { Factors affecting rural patients' primary } \\
\text { compliance with e-prescription: A developing } \\
\text { country perspective }\end{array}$ & Telemedicine and e-Health & 1.996 & 2019 & 2 & 102.00 \\
\hline AlBar, A.M.; Hoque, M.R. & $\begin{array}{l}\text { Patient acceptance of e-health services in Saudi } \\
\text { Arabia: An integrative perspective }\end{array}$ & Telemedicine and e-Health & 1.996 & 2019 & 2 & 102.00 \\
\hline $\begin{array}{l}\text { Treskes, R.; Wildbergh, T.; Schalij, M.; } \\
\text { Scherptong, R. }\end{array}$ & $\begin{array}{l}\text { Expectations and perceived barriers to widespread } \\
\text { implementation of e-health in cardiology practice: } \\
\text { Results from a national survey in the Netherlands }\end{array}$ & Neth. Heart J. & 1.972 & 2019 & 2 & 102.00 \\
\hline $\begin{array}{l}\text { Koole, M.A.C.; Kauw, D.; Winter, } \\
\text { M.M.; Dohmen, D.A.J.; Tulevski, I.I; } \\
\text { de Haan, R.; Somsen, G.A.; Schijven, } \\
\text { M.P.; Robbers-Visser, D.; Mulder, } \\
\text { B.J.M.; Bouma, B.J.; Schuuring, M.J. }\end{array}$ & $\begin{array}{l}\text { First real-world experience with mobile health } \\
\text { telemonitoring in adult patients with congenital } \\
\text { heart disease }\end{array}$ & Neth. Heart J. & 1.972 & 2019 & 2 & 102.00 \\
\hline $\begin{array}{l}\text { Kim, S.C.; Shaw, B.R.; Shah, D.V.; } \\
\text { Hawkins, R.P.; Pingree, S.; McTavish, } \\
\text { F.M.; Gustafson, D.H. }\end{array}$ & $\begin{array}{l}\text { Interactivity, presence, and targeted patient care: } \\
\text { Mapping e-health intervention effects over time for } \\
\text { cancer patients with depression }\end{array}$ & Health Communication & 1.846 & 2019 & 2 & 102.00 \\
\hline Hang, L.; Choi, E.; Kim, D.H. & $\begin{array}{l}\text { A novel EMR integrity management based on a } \\
\text { medical blockchain platform in hospital }\end{array}$ & Electronics & 1.764 & 2019 & 2 & 102.00 \\
\hline Dao, J.; Spooner, C.; Lo, W.; Harris, M. & $\begin{array}{c}\text { Factors influencing self-management in patients } \\
\text { with type } 2 \text { diabetes in general practice: A } \\
\text { qualitative study }\end{array}$ & Australian Journal of Primary Health & 1.024 & 2019 & 2 & 102.00 \\
\hline $\begin{array}{c}\text { Lee, H.; Sullivan, S.J.; Schneiders, } \\
\text { A.G.; Ahmed, O.H.; Balasundaram, } \\
\text { A.P.; Williams, D.; Meeuwisse, W.H.; } \\
\text { McCrory, P. }\end{array}$ & $\begin{array}{l}\text { Smartphone and tablet apps for concussion road } \\
\text { warriors (team clinicians): A systematic review for } \\
\text { practical users }\end{array}$ & Br. J. Sports Med. & 0 & 2015 & 42 & 102.00 \\
\hline $\begin{array}{l}\text { [95] Kebede, M.; Peters, M.; Heise, T.; } \\
\text { Pischke, C. }\end{array}$ & $\begin{array}{l}\text { Comparison of three meta-analytic methods using } \\
\text { data from digital interventions on type } 2 \text { diabetes }\end{array}$ & $\begin{array}{l}\text { Diabetes, Metabolic Syndrome and } \\
\text { Obesity }\end{array}$ & 0 & 2019 & 2 & 102.00 \\
\hline $\begin{array}{l}\text { Kooistra, L.; Ruwaard, J.; Wiersma, J.; } \\
\text { Van Oppen, P.; Van Der Vaart, R.; Van } \\
\text { Gemert-Pijnen, J.; Riper, H. }\end{array}$ & $\begin{array}{l}\text { Development and initial evaluation of blended } \\
\text { cognitive behavioural treatment for major } \\
\text { depression in routine specialized mental } \\
\text { health care }\end{array}$ & Internet Interv. & 0 & 2016 & 32 & 102.00 \\
\hline $\begin{array}{c}\text { Petrakis, E.G.; Sotiriadis, S.; } \\
\text { Soultanopoulos, T; Renta, P.T.; Buyya, } \\
\text { R.; Bessis, N. }\end{array}$ & $\begin{array}{l}\text { Internet of Things as a Service (iTaaS): Challenges } \\
\text { and solutions for management of sensor data on } \\
\text { the cloud and the fog }\end{array}$ & Internet of Things & 0 & 2018 & 12 & 102.00 \\
\hline Rajan, S.P. & $\begin{array}{l}\text { Review and investigations on future research } \\
\text { directions of mobile based telecare system for } \\
\text { cardiac surveillance }\end{array}$ & J. Appl. Res. Technol. & 0 & 2015 & 42 & 102.00 \\
\hline $\begin{array}{l}\text { [65] Bricker, J.; Mull, K.; McClure, J.; } \\
\text { Watson, N.; Heffner, J. }\end{array}$ & $\begin{array}{l}\text { Improving quit rates of web-delivered } \\
\text { interventions for smoking cessation: Full-scale } \\
\text { randomized trial of WebQuit.org versus } \\
\text { Smokefree.gov }\end{array}$ & Addiction & 6.851 & 2018 & 11 & 101.01 \\
\hline $\begin{array}{c}\text { Van Den Berg, M.; Crotty, M.; Liu, E.; } \\
\text { Killington, M.; Kwakkel, G.; Van } \\
\text { Wegen, E. }\end{array}$ & $\begin{array}{l}\text { Early supported discharge by caregiver-mediated } \\
\text { exercises and e-health support after stroke: A } \\
\text { proof-of-concept trial }\end{array}$ & Stroke & 6.046 & 2016 & 31 & 101.01 \\
\hline Al-Sharhan, S.; Omran, E.; Lari, K. & $\begin{array}{l}\text { An integrated holistic model for an eHealth system: } \\
\text { A national implementation approach and a new } \\
\text { cloud-based security model }\end{array}$ & $\begin{array}{c}\text { International Journal of Information } \\
\text { Management }\end{array}$ & 5.063 & 2019 & 1 & 101.01 \\
\hline [82] Liu, X., Zhou, Y.; Wang, Z. & $\begin{array}{l}\text { Can the development of a patient's condition be } \\
\text { predicted through intelligent inquiry under the } \\
\text { e-health business mode? Sequential feature } \\
\text { map-based disease risk prediction upon features } \\
\text { selected from cognitive diagnosis Big Data }\end{array}$ & Int. J. Inf. Manag. & 5.063 & 2019 & 1 & 101.01 \\
\hline $\begin{array}{l}\text { Danaher, B.; Tyler, M.; Crowley, R.; } \\
\text { Brendryen, H.; Seeley, J. }\end{array}$ & $\begin{array}{l}\text { Outcomes and device usage for fully automated } \\
\text { internet interventions designed for a smartphone } \\
\text { or personal computer: The MobileQuit smoking } \\
\text { cessation randomized controlled trial }\end{array}$ & J. Med. Internet Res. & 4.945 & 2019 & 1 & 101.00 \\
\hline $\begin{array}{l}\text { Din, H.; Mcdaniels-Davidson, C.; } \\
\text { Nodora, J.; Madanat, H. }\end{array}$ & $\begin{array}{l}\text { Profiles of a health information-seeking population } \\
\text { and the current digital divide: Cross-sectional } \\
\text { analysis of the 2015-2016 California health } \\
\text { interview survey }\end{array}$ & J. Med. Internet Res. & 4.945 & 2019 & 1 & 101.00 \\
\hline $\begin{array}{l}\text { Holter, M.; Johansen, A.; Ness, O.; } \\
\text { Brinkmann, S.; Hoybye, M.; } \\
\text { Brendryen, H. }\end{array}$ & $\begin{array}{l}\text { Qualitative interview studies of working } \\
\text { mechanisms in electronic health: tools to enhance } \\
\text { study quality }\end{array}$ & J. Med. Internet Res. & 4.945 & 2019 & 1 & 101.00 \\
\hline $\begin{array}{l}\text { Kuipers, E.; Poot, C.C.; Wensing, M.; } \\
\text { Chavannes, N.H.; de Smet, P.A.G.M.; } \\
\text { Teichert, M. }\end{array}$ & $\begin{array}{l}\text { Self-Management Maintenance Inhalation Therapy } \\
\text { with eHealth (SELFIE): Observational study on the } \\
\text { use of an electronic monitoring device in } \\
\text { respiratory patient care and research }\end{array}$ & J. Med. Internet Res. & 4.945 & 2019 & 1 & 101.00 \\
\hline $\begin{array}{l}\text { Hsia, T.-L.; Chiang, A.J.; Wu, J.-H.; } \\
\text { Teng, N.N.; Rubin, A.D. }\end{array}$ & $\begin{array}{l}\text { What drives e-health usage? Integrated } \\
\text { institutional forces and top } \\
\text { management perspectives }\end{array}$ & Computers in Human Behavior & 4.306 & 2019 & 1 & 101.00 \\
\hline
\end{tabular}


Table A1. Cont.

\begin{tabular}{|c|c|c|c|c|c|c|}
\hline Author & Title & Journal & $\begin{array}{l}\text { Impact } \\
\text { Factor }\end{array}$ & Year & Citations & InOrdinatio 10 \\
\hline Klaib, A.F.; Nuser, M.S. & $\begin{array}{l}\text { Evaluating EHR and health care in Jordan } \\
\text { according to the international Health Metrics } \\
\text { Network (HMN) framework and standards: A case } \\
\text { study of Hakeem }\end{array}$ & Access, IEEE & 4.098 & 2019 & 1 & 101.00 \\
\hline $\begin{array}{l}\text { Menon, A.; Gray, L.; Fatehi, F; Bird, } \\
\text { D.; Darssan, D.; Karunanithi, M.; } \\
\text { Russell, A. }\end{array}$ & $\begin{array}{l}\text { Mobile-based insulin dose adjustment for type } 2 \\
\text { diabetes in community and rural populations: } \\
\text { Study protocol for a pilot randomized } \\
\text { controlled trial }\end{array}$ & Ther. Adv. Endocrinol. Metab. & 3.543 & 2019 & 1 & 101.00 \\
\hline Smith, B.; Magnani, J. & $\begin{array}{l}\text { New technologies, new disparities: The } \\
\text { intersection of electronic health and digital } \\
\text { health literacy }\end{array}$ & International Journal of Cardiology & 3.471 & 2019 & 1 & 101.00 \\
\hline $\begin{array}{l}\text { Gibbs, J.; Gkatzidou, V.; Tickle, L.; } \\
\text { Manning, S.; Tilakkumar, T.; Hone, K.; } \\
\text { Ashcroft, R.; Sonnenberg, P.; Sadiq, S.; } \\
\text { Estcourt, C. }\end{array}$ & $\begin{array}{l}\text { “Can you recommend any good STI apps?" A } \\
\text { review of content, accuracy and } \\
\text { comprehensiveness of current mobile medical } \\
\text { applications for STIs and related genital infections }\end{array}$ & Sex Transm Infect & 3.365 & 2017 & 21 & 101.00 \\
\hline $\begin{array}{l}\text { Heard, K.; Hughes, S.; Mughal, N.; } \\
\text { Azadian, B.; Moore, L. }\end{array}$ & $\begin{array}{l}\text { Evaluating the impact of the ICNET }{ }^{\circledR} \text { clinical } \\
\text { decision support system for antimicrobial } \\
\text { stewardship }\end{array}$ & $\begin{array}{l}\text { Antimicrobial Resistance and } \\
\text { Infection Control }\end{array}$ & 3.224 & 2019 & 1 & 101.00 \\
\hline $\begin{array}{l}\text { [91] Wild, T.; Fromberger, P.; Jordan, } \\
\text { K.; Müller, I.; Müller, J. }\end{array}$ & $\begin{array}{l}\text { Web-based health services in forensic psychiatry: A } \\
\text { review of the use of the internet in the treatment of } \\
\text { child sexual abusers and child sexual exploitation } \\
\text { material offenders }\end{array}$ & Front Psychiatry & 3.164 & 2019 & 1 & 101.00 \\
\hline $\begin{array}{l}\text { Miramontes, R.; Aquino, R.; Flores, A.; } \\
\text { Rodríguez, G.; Anguiano, R., Ríos, A.; } \\
\text { Edwards, A. }\end{array}$ & $\begin{array}{l}\text { PlaIMoS: A remote mobile healthcare platform to } \\
\text { monitor cardiovascular and respiratory variables }\end{array}$ & Sensors & 3.031 & 2017 & 21 & 101.00 \\
\hline Woods, S.; Sullivan, K. & $\begin{array}{l}\text { Lower neurocognitive functioning disrupts the } \\
\text { effective use of internet-based health resources in } \\
\text { HIV disease: the mediating effects of general health } \\
\text { literacy capacity }\end{array}$ & AIDS Behav. & 2.908 & 2019 & 1 & 101.00 \\
\hline $\begin{array}{l}\text { Young, C.; Campolonghi, S.; } \\
\text { Ponsonby, S.; Dawson, S.L.; O'Neil, A.; } \\
\text { Kay-Lambkin, F.; McNaughton, S.A.; } \\
\text { Berk, M.; Jacka, F.N. }\end{array}$ & $\begin{array}{l}\text { Supporting engagement, adherence, and behavior } \\
\text { change in online dietary interventions }\end{array}$ & $\begin{array}{l}\text { Journal of Nutrition Education and } \\
\text { Behavior }\end{array}$ & 2.869 & 2019 & 1 & 101.00 \\
\hline $\begin{array}{l}\text { Walsh, K; Pryor, T.; Reynolds, K.; } \\
\text { Walker, J. }\end{array}$ & $\begin{array}{l}\text { Searching for answers: How well do depression } \\
\text { websites answer the public's questions about } \\
\text { treatment choices? }\end{array}$ & Patient Educ. Couns. & 2.821 & 2019 & 1 & 101.00 \\
\hline Cipolletta, S.; Mocellin, D. & $\begin{array}{l}\text { Online counseling: An exploratory survey of } \\
\text { Italian psychologists' attitudes towards new ways } \\
\text { of interaction }\end{array}$ & Psychother. Res. & 2.788 & 2018 & 11 & 101.00 \\
\hline $\begin{array}{c}\text { Olivero, E.; Bert, F.; Thomas, R.; } \\
\text { Scarmozzino, A.; Raciti, I.; Gualano, } \\
\text { M.; Siliquini, R. }\end{array}$ & $\begin{array}{l}\text { E-tools for hospital management: An overview of } \\
\text { smartphone applications for health professionals }\end{array}$ & $\begin{array}{l}\text { International Journal of Medical } \\
\text { Informatics }\end{array}$ & 2.721 & 2019 & 1 & 101.00 \\
\hline $\begin{array}{l}\text { Calvillo-Arbizu, J.; Roa-Romero, L.M.; } \\
\text { Estudillo-Valderrama, M.A.; } \\
\text { Salgueira-Lazo, M.; Aresté-Fosalba, } \\
\text { N.; Del Castillo-Rodríguez, N.L.; } \\
\text { González-Cabrera, F.; } \\
\text { Marrero-Robayna, S.; De-La Manzana, } \\
\text { V.L.; Román-Martínez, I. }\end{array}$ & $\begin{array}{l}\text { User-centred design for developing e-health } \\
\text { system for renal patients at home (AppNephro) }\end{array}$ & $\begin{array}{l}\text { International Journal of Medical } \\
\text { Informatics }\end{array}$ & 2.721 & 2019 & 1 & 101.00 \\
\hline Haddad, S.; Souza, R.; Cecatti, J. & $\begin{array}{l}\text { Mobile technology in health (mHealth) and } \\
\text { antenatal care-searching for apps and available } \\
\text { solutions: A systematic review }\end{array}$ & $\begin{array}{l}\text { International Journal of Medical } \\
\text { Informatics }\end{array}$ & 2.721 & 2019 & 1 & 101.00 \\
\hline $\begin{array}{l}\text { Miñarro-Giménez, J.; Cornet, R.; } \\
\text { Jaulent, M.; Dewenter, H.; Thun, S., } \\
\text { Gøeg, K.; Karlsson, D.; Schulz, S. }\end{array}$ & $\begin{array}{l}\text { Quantitative analysis of manual annotation of } \\
\text { clinical text samples }\end{array}$ & $\begin{array}{l}\text { International Journal of Medical } \\
\text { Informatics }\end{array}$ & 2.721 & 2019 & 1 & 101.00 \\
\hline $\begin{array}{l}\text { Lauridsen, S.; Braae, U.C.; Ngowi, } \\
\text { H.A.; Johansen, M.V. }\end{array}$ & $\begin{array}{c}\text { Impacts of using the electronic-health education } \\
\text { program "The Vicious Worm" for prevention of } \\
\text { Taenia solium }\end{array}$ & Acta Tropica & 2.629 & 2019 & 1 & 101.00 \\
\hline $\begin{array}{c}\text { Heger, I; Deckers, K.; Van Boxtel, M.; } \\
\text { De Vugt, M.; Hajema, K., Verhey, F.; } \\
\text { Köhler, S. }\end{array}$ & $\begin{array}{l}\text { Dementia awareness and risk perception in } \\
\text { middle-aged and older individuals: Baseline } \\
\text { results of the MijnBreincoach survey on the } \\
\text { association between lifestyle and brain health }\end{array}$ & BMC Public Health & 2.567 & 2019 & 1 & 101.00 \\
\hline $\begin{array}{l}\text { Kloek, C.; Van Tilburg, M.; Staal, J.; } \\
\text { Veenhof, C.; Bossen, D. }\end{array}$ & $\begin{array}{l}\text { Development and proof of concept of a blended } \\
\text { physiotherapeutic intervention for patients with } \\
\text { non-specific low back pain }\end{array}$ & Physiotherapy (United Kingdom) & 2.534 & 2019 & 1 & 101.00 \\
\hline Novak, S.; Djordjevic, N. & $\begin{array}{l}\text { Information system for evaluation of healthcare } \\
\text { expenditure and health monitoring }\end{array}$ & Physica A & 2.500 & 2019 & 1 & 101.00 \\
\hline $\begin{array}{l}\text { [101] Amin, R.; Islam, S.K.H.; Biswas, } \\
\text { G.P.; Khan, M.K.; Li, X. }\end{array}$ & $\begin{array}{l}\text { Cryptanalysis and enhancement of anonymity } \\
\text { preserving remote user mutual authentication and } \\
\text { session key agreement scheme for e-health } \\
\text { care systems }\end{array}$ & J. Med. Syst. & 2.415 & 2015 & 41 & 101.00 \\
\hline $\begin{array}{l}\text { [51] Li, X.; Niu, J.; Karuppiah, M.; } \\
\text { Kumari, S.; Wu, F. }\end{array}$ & $\begin{array}{l}\text { Secure and efficient two-factor user authentication } \\
\text { scheme with user anonymity for network based } \\
\text { e-health care applications }\end{array}$ & J. Med. Syst. & 2.415 & 2016 & 31 & 101.00 \\
\hline $\begin{array}{l}\text { Wiljer, D.; Charow, R.; Costin, H.; } \\
\text { Sequeira, L;; Anderson, M., } \\
\text { Strudwick, G.; Tripp, T.; Crawford, A. }\end{array}$ & $\begin{array}{l}\text { Defining compassion in the digital health age: } \\
\text { Protocol for a scoping review }\end{array}$ & BMJ Open & 2.376 & 2019 & 1 & 101.00 \\
\hline $\begin{array}{l}\text { Mangin, D.; Parascandalo, J., } \\
\text { Khudoyarova, O., Agarwal, G., } \\
\text { Bismah, V.; Orr, S. }\end{array}$ & $\begin{array}{l}\text { Multimorbidity, eHealth and implications for } \\
\text { equity: A cross-sectional survey of patient } \\
\text { perspectives on eHealth }\end{array}$ & BMJ Open & 2.376 & 2019 & 1 & 101.00 \\
\hline $\begin{array}{l}\text { Krusche, A.; Bradbury, K.; Corbett, T.; } \\
\text { Barnett, J.; Stuart, B.; Yao, G.L.; Bacon, } \\
\text { R.; Böhning, D.; Cheetham-Blake, T.; } \\
\text { Eccles, D.; Foster, C.; Geraghty, } \\
\text { A.W.A.; Leydon, G.; Müller, A.; Neal, } \\
\text { R.D.; Osborne, R.; Rathod, S.; } \\
\text { Richardson, A.; Sharman, G.; } \\
\text { Summers, K.; Watson, E.; Wilde, L.; } \\
\text { Wilkinson, C.; Yardley, L.; Little, P. }\end{array}$ & $\begin{array}{l}\text { Renewed: Protocol for a randomised controlled } \\
\text { trial of a digital intervention to support quality of } \\
\text { life in cancer survivors }\end{array}$ & BMJ Open & 2.376 & 2019 & 1 & 101.00 \\
\hline
\end{tabular}


Table A1. Cont.

\begin{tabular}{|c|c|c|c|c|c|c|}
\hline Author & Title & Journal & $\begin{array}{l}\text { Impact } \\
\text { Factor }\end{array}$ & Year & Citations & InOrdinatio 10 \\
\hline Zayyad, M.A.; Toycan, M. & $\begin{array}{l}\text { Factors affecting sustainable adoption of e-health } \\
\text { technology in developing countries: An } \\
\text { exploratory survey of Nigerian hospitals from the } \\
\text { perspective of healthcare professionals }\end{array}$ & PEERJ & 2.353 & 2018 & 11 & 101.00 \\
\hline Zanutto, A. & $\begin{array}{l}\text { "Two clicks and I'm in!" Patients as co-actors in } \\
\text { managing health data through a personal health } \\
\text { record infrastructure }\end{array}$ & Health Informatics $J$. & 2.297 & 2019 & 1 & 101.00 \\
\hline Mauco, K.L.; Scott, R.E.; Mars, M. & $\begin{array}{l}\text { Critical analysis of e-health readiness assessment } \\
\text { frameworks: Suitability for application in } \\
\text { developing countries }\end{array}$ & J. Telemed. Telecare & 2.229 & 2018 & 11 & 101.00 \\
\hline $\begin{array}{l}\text { Olayiwola, J.N.; Potapov, A.; Gordon, } \\
\text { A.; Jurado, J.; Magana, C.; Knox, M.; } \\
\text { Tuot, D. }\end{array}$ & $\begin{array}{l}\text { Electronic consultation impact from the primary } \\
\text { care clinician perspective: Outcomes from a } \\
\text { national sample }\end{array}$ & J. Telemed. Telecare & 2.229 & 2019 & 1 & 101.00 \\
\hline $\begin{array}{l}\text { [103] Kompara, M.; Kumari, S.; Hölbl, } \\
\text { M. }\end{array}$ & $\begin{array}{l}\text { Analysis and improvement of a secure key } \\
\text { management protocol for e-health applications }\end{array}$ & Computers \& Electrical Engineering & 2.189 & 2019 & 1 & 101.00 \\
\hline $\begin{array}{l}\text { Nymberg, V.M.; Bolmsjo, B.B.; Wolff, } \\
\text { M.; Calling, S.; Gerward, S.; } \\
\text { Sandberg, M. }\end{array}$ & $\begin{array}{l}\text { "Having to learn this so late in our lives horizontal } \\
\text {..." Swedish elderly patients' beliefs, experiences, } \\
\text { attitudes and expectations of e-health in primary } \\
\text { health care }\end{array}$ & Scand J Prim Health Care & 2.095 & 2019 & 1 & 101.00 \\
\hline $\begin{array}{l}\text { Gupta, S.; Cheung, V.L.S.; Kastner, M.; } \\
\text { Straus, S.; Kaplan, A.; Boulet, L.-P.; } \\
\text { Sale, J.E.M. }\end{array}$ & $\begin{array}{l}\text { Patient preferences for a touch screen tablet-based } \\
\text { asthma questionnaire }\end{array}$ & Journal of Asthma & 2.081 & 2019 & 1 & 101.00 \\
\hline $\begin{array}{l}\text { James, N.; Power, E.; Hogden, A.; } \\
\text { Vucic, S. }\end{array}$ & $\begin{array}{l}\text { Patients' perspectives of multidisciplinary } \\
\text { home-based e-health service delivery for motor } \\
\text { neurone disease }\end{array}$ & Disability and rehabilitation & 2.054 & 2019 & 1 & 101.00 \\
\hline $\begin{array}{l}\text { Dang, S.; Ruiz, D.; Klepac, L.; Morse, } \\
\text { S.; Becker, P.; Levy, C.; Kinosian, B.; } \\
\text { Edes, T. }\end{array}$ & $\begin{array}{l}\text { Key characteristics for successful adoption and } \\
\text { implementation of home telehealth technology in } \\
\text { Veterans Affairs home-based primary care: An } \\
\text { exploratory study }\end{array}$ & Telemedicine and $\mathrm{e}$-Health & 1.996 & 2019 & 1 & 101.00 \\
\hline Botrugno, C. & Towards an ethics for telehealth & Nurs Ethics & 1.957 & 2019 & 1 & 101.00 \\
\hline Petrellis, N.; Birbas, M.; Gioulekas, F. & $\begin{array}{l}\text { On the design of low-cost IoT sensor node for } \\
\text { e-health environments }\end{array}$ & Electronics & 1.764 & 2019 & 1 & 101.00 \\
\hline Shin, S.Y. & $\begin{array}{l}\text { Current status and future direction of digital health } \\
\text { in Korea }\end{array}$ & Korean J. Physiol. Pharmacol & 1.654 & 2019 & 1 & 101.00 \\
\hline $\begin{array}{l}\text { Van Dooren, M.M.; Siriaraya, P.; Visch, } \\
\text { V.; Spijkerman, R.; Bijkerk, L. }\end{array}$ & $\begin{array}{l}\text { Reflections on the design, implementation, and } \\
\text { adoption of a gamified eHealth application in } \\
\text { youth mental healthcare }\end{array}$ & Entertainment Computing & 1.297 & 2019 & 1 & 101.00 \\
\hline $\begin{array}{l}\text { Kinnunen, U.-M.; Heponiemi, T.; } \\
\text { Rajalahti, E.; Ahonen, O.; Korhonen, } \\
\text { T.; Hyppönen, H. }\end{array}$ & $\begin{array}{l}\text { Factors related to health informatics competencies } \\
\text { for nurses-results of a national electronic health } \\
\text { record survey }\end{array}$ & $\begin{array}{l}\text { CIN-Computers Informatics } \\
\text { Nursing }\end{array}$ & 1.029 & 2019 & 1 & 101.00 \\
\hline $\begin{array}{l}\text { Del Río Carral, M.; Schweizer, A.; } \\
\text { Papon, A.; Santiago-Delefosse, M. }\end{array}$ & $\begin{array}{l}\text { Connected objects and health applications: } \\
\text { Exploratory study on attitudes, use (or non-use) } \\
\text { and contexts of use [Les objets connectés et } \\
\text { applications de sante: étude exploratoire des } \\
\text { perceptions, usages (ou non) et contextes d'usage] }\end{array}$ & Pratiques Psychologiques & 0.196 & 2019 & 1 & 101.00 \\
\hline Kiberu, V.M.; Mars, M.; Scott, R.E. & $\begin{array}{l}\text { Barriers and opportunities to implementation of } \\
\text { sustainable e-health programmes in Uganda: A } \\
\text { literature review }\end{array}$ & AFR. J. Prim. Health Care Fam. Med. & 0 & 2017 & 21 & 101.00 \\
\hline $\begin{array}{l}\text { Delgado, J.A.M.; Alonso, F.J.M.; } \\
\text { Boquet, E.M.; de Tomás, J.F.Â.; } \\
\text { Díez, J.M.C. }\end{array}$ & $\begin{array}{l}\text { Competencias digitales clave de los } \\
\text { profesionales sanitarios }\end{array}$ & Educación Médica & 0 & 2019 & 1 & 101.00 \\
\hline $\begin{array}{l}\text { Dai, J.; Granikov, V.; El Sherif, R.; } \\
\text { Grguric, E.; Turcotte, E.; Pluye, P. }\end{array}$ & $\begin{array}{l}\text { Patient Information Aid: An innovative } \\
\text { educational program to improve outcomes of } \\
\text { online consumer health information }\end{array}$ & Education For Information & 0 & 2019 & 1 & 101.00 \\
\hline Kose, T;; Oymak, C. & $\begin{array}{l}\text { E-health in Turkey: An analysis of } \\
\text { consumer activities }\end{array}$ & Health and Technology & 0 & 2019 & 1 & 101.00 \\
\hline $\begin{array}{l}\text { [100] Ramu, G.; Reddy, B.E.; Jayanthi, } \\
\text { A.; Prasad, L.V.N. }\end{array}$ & $\begin{array}{l}\text { Fine-grained access control of EHRs in cloud using } \\
\text { CP-ABE with user revocation }\end{array}$ & Health and Technology & 0 & 2019 & 1 & 101.00 \\
\hline $\begin{array}{l}\text { Merlo, C.; Akle, A.A.; Llaria, A.; } \\
\text { Terrasson, G.; Villeneuve, E.; } \\
\text { Pilnière, V. }\end{array}$ & $\begin{array}{l}\text { Proposal of a user-centred approach for CPS } \\
\text { design: Pillbox case study }\end{array}$ & IFAC-PapersOnLine & 0 & 2019 & 1 & 101.00 \\
\hline $\begin{array}{l}\text { Omotosho, A.; Ayegba, P.; } \\
\text { Emuoyibofarhe, J.; Meinel, C. }\end{array}$ & $\begin{array}{l}\text { Current state of ICT in healthcare delivery in } \\
\text { developing countries }\end{array}$ & $\begin{array}{l}\text { International Journal of Online and } \\
\text { Biomedical Engineering }\end{array}$ & 0 & 2019 & 1 & 101.00 \\
\hline $\begin{array}{l}\text { Van Der Meulen, H.; Mccashin, D.; } \\
\text { O'Reilly, G.; Coyle, D. }\end{array}$ & $\begin{array}{l}\text { Using computer games to support mental health } \\
\text { interventions: Naturalistic deployment study }\end{array}$ & JMIR Mental Health & 0 & 2019 & 1 & 101.00 \\
\hline $\begin{array}{l}\text { Moor, C.; Gür-Demirel, Y.; } \\
\text { Wijsenbeek, M. }\end{array}$ & $\begin{array}{l}\text { Feasibility of a comprehensive home monitoring } \\
\text { program for sarcoidosis }\end{array}$ & J. Pers. Med. & 0 & 2019 & 1 & 101.00 \\
\hline $\begin{array}{l}\text { Maunder, K.; Walton, K.; Williams, P.; } \\
\text { Ferguson, M.; Beck, E. }\end{array}$ & $\begin{array}{l}\text { Strategic leadership will be essential for dietitian } \\
\text { eHealth readiness: A qualitative study exploring } \\
\text { dietitian perspectives of eHealth readiness }\end{array}$ & $\begin{array}{l}\text { Nutrition \& Dietetics: The Journal of } \\
\text { The Dietitians Association of } \\
\text { Australia }\end{array}$ & 0 & 2019 & 1 & 101.00 \\
\hline $\begin{array}{l}\text { Naarding, P; Marijnissen, R.; } \\
\text { Westerhof, G. }\end{array}$ & Digital psychiatry [Digitale psychiatrie] & Tijdschr. Psychiatr. & 0 & 2019 & 1 & 101.00 \\
\hline $\begin{array}{l}\text { Licari, A.; Ferrante, G.; Gian Luigi } \\
\text { Marseglia, M.D.; Giovanni Corsello, } \\
\text { M.D.; Grutta, S.L. }\end{array}$ & $\begin{array}{l}\text { What is the impact of innovative electronic health } \\
\text { interventions in improving treatment adherence in } \\
\text { asthma? The pediatric perspective }\end{array}$ & J. Allergy Clin. Immunol. Pract. & 7.550 & 2019 & 0 & 100.01 \\
\hline $\begin{array}{l}\text { Garcia, S.; Wortman, K.; Cella, D.; } \\
\text { Wagner, L.; Bass, M.; Kircher, S.; } \\
\text { Pearman, T.; Penedo, F. }\end{array}$ & $\begin{array}{l}\text { Implementing electronic health record-integrated } \\
\text { screening of patient-reported symptoms and } \\
\text { supportive care needs in a comprehensive } \\
\text { cancer center }\end{array}$ & Cancer & 6.164 & 2019 & 0 & 100.01 \\
\hline $\begin{array}{l}\text { Carter, J.; Seed, P.T.; Watson, H.A.; } \\
\text { David, A.L.; Sandall, J.; Shennan, } \\
\text { A.H.; Tribe, R.M. }\end{array}$ & $\begin{array}{l}\text { Development and validation of prediction models } \\
\text { for the QUiPP App v.2: A tool for predicting } \\
\text { preterm birth in women with symptoms of } \\
\text { threatened preterm labor }\end{array}$ & $\begin{array}{l}\text { Ultrasound in obstetrics \& } \\
\text { gynecology }\end{array}$ & 5.595 & 2019 & 0 & 100.01 \\
\hline
\end{tabular}


Table A1. Cont.

\begin{tabular}{|c|c|c|c|c|c|c|}
\hline Author & Title & Journal & $\begin{array}{l}\text { Impact } \\
\text { Factor }\end{array}$ & Year & Citations & InOrdinatio 10 \\
\hline $\begin{array}{l}\text { Delanty, N.; White, M.; Benson, K.; } \\
\text { McCormack, M.; Heavin,, S.; } \\
\text { Comerford, E.; Gangadharan, N.; } \\
\text { Power, K.; Dunleavey, B.; El-Naggar, } \\
\text { H.; Doherty, C.; Greally, M.; Cavalleri, } \\
\text { G.; Fitzsimons, M. }\end{array}$ & $\begin{array}{l}\text { Development of a genomics module within an } \\
\text { epilepsy-specific electronic health record: Toward } \\
\text { genomic medicine in epilepsy care }\end{array}$ & Epilepsia & 5.562 & 2019 & 0 & 100.01 \\
\hline $\begin{array}{l}\text { Webber, E;; Brick, D.; Scibiliaa, J.; } \\
\text { Dehnel, P.; Weinberg, S.; Alexander, } \\
\text { G.; Beyer, E.; Hamling, A.; Kirkendall, } \\
\text { E.; Lighter, D.; Mann, A.; Morgan, S.; } \\
\text { Shelov, E.; Wright, J.; Alverson, D.; } \\
\text { Chan, F.; Van Cain, M.; Krams, L.; } \\
\text { Altman, R.; Bondi, S.; Fanaroff, J.; } \\
\text { Narang, S.; Oken, R.; Rusher, J.; } \\
\text { Santucci, K.; Scott, S.; Ake, J.; } \\
\text { Alexander, J.; Bodnar, C.; Curfman, A., } \\
\text { Herendeen, N.; Kahn, J.; McSwain, S.; } \\
\text { Garber, K.; Calabrese, T., Council On } \\
\text { Clinical Information Technology; } \\
\text { Committee On Medical Liability And } \\
\text { Risk Management; Section On } \\
\text { Telehealth Care. }\end{array}$ & $\begin{array}{l}\text { Electronic communication of the health record and } \\
\text { information with pediatric patients and } \\
\text { their guardians }\end{array}$ & Pediatrics & 5.401 & 2019 & 0 & 100.01 \\
\hline Yi, C.; Cai, J. & $\begin{array}{l}\text { Delay-dependent priority-aware transmission } \\
\text { scheduling for e-health networks: A mechanism } \\
\text { design approach }\end{array}$ & IEEE Trans. Veh. Technol. & 5.339 & 2019 & 0 & 100.01 \\
\hline $\begin{array}{l}\text { Fang, S.H.; Li, C.C.; Lu, W.C.; Xu, Z.; } \\
\text { Chien, Y.R. }\end{array}$ & $\begin{array}{l}\text { Enhanced device-free human detection: Efficient } \\
\text { learning from phase and amplitude of channel } \\
\text { state information }\end{array}$ & IEEE Trans. Veh. Technol. & 5.339 & 2019 & 0 & 100.01 \\
\hline $\begin{array}{l}\text { Elfstrom, K.M.; Sundstrom, K.; } \\
\text { Andersson, S.; Bzhalava, Z.; Carlsten } \\
\text { Thor, A.; Gzoul, Z; Ohman, D.; Lamin, } \\
\text { H.; Eklund, C.; Dillner, J; Tornberg, S. }\end{array}$ & $\begin{array}{l}\text { Increasing participation in cervical screening by } \\
\text { targeting long-term nonattenders: Randomized } \\
\text { health services study }\end{array}$ & International Journal of Cancer & 4.982 & 2019 & 0 & 100.00 \\
\hline $\begin{array}{l}\text { Cullinan, N.; Villani, A.; Mourad, S.; } \\
\text { Somers, G.; Reichman, L; Van } \\
\text { Engelen, K.; Stephens, D.; Weksberg, } \\
\text { R.; Foulkes, W.; Malkin, D.; Grant, R; } \\
\text { Goudie, C. }\end{array}$ & $\begin{array}{l}\text { An eHealth decision-support tool to prioritize } \\
\text { referral practices for genetic evaluation of patients } \\
\text { with Wilms tumor }\end{array}$ & International Journal of Cancer & 4.982 & 2019 & 0 & 100.00 \\
\hline $\begin{array}{c}\text { Den Bakker, C.M.; Huirne, J.A.; } \\
\text { Schaafsma, F.G.; De Geus, C., Bonjer, } \\
\text { H.J.; Anema, J.R. }\end{array}$ & $\begin{array}{l}\text { Electronic health program to empower patients in } \\
\text { returning to normal activities after colorectal } \\
\text { surgical procedures: Mixed-methods process } \\
\text { evaluation alongside a randomized controlled trial }\end{array}$ & J. Med. Internet Res. & 4.945 & 2019 & 0 & 100.00 \\
\hline $\begin{array}{l}\text { Paulsen, M.; Varsi, C.; Paur, L.; } \\
\text { Tangvik, R.; Andersen, L. }\end{array}$ & $\begin{array}{l}\text { Barriers and facilitators for implementing a } \\
\text { decision support system to prevent and treat } \\
\text { disease-related malnutrition in a hospital setting: } \\
\text { Qualitative study }\end{array}$ & J. Med. Internet Res. & 4.945 & 2019 & 0 & 100.00 \\
\hline $\begin{array}{l}\text { Ariens, L.F.M.; Schussler-Raymakers, } \\
\text { F.M.L.; Frima, C.; Flinterman, A.; } \\
\text { Hamminga, E.; Arents, B.W.M.; } \\
\text { Bruijnzeel-Koomen, C.A.F.M.; De } \\
\text { Bruin-Weller, M.S.; Van } \\
\text { Os-Medendorp, H. }\end{array}$ & $\begin{array}{l}\text { Barriers and facilitators to eHealth use in daily } \\
\text { practice: Perspectives of patients and professionals } \\
\text { in dermatology }\end{array}$ & J. Med. Internet Res. & 4.945 & 2017 & 20 & 100.00 \\
\hline $\begin{array}{l}\text { Ijzerman, R.V.H.; Van Der Vaart, R.; } \\
\text { Evers, A.W.M. }\end{array}$ & $\begin{array}{l}\text { Internet-based cognitive behavioral therapy among } \\
\text { psychologists in a medical setting: A survey } \\
\text { on implementation }\end{array}$ & J. Med. Internet Res. & 4.945 & 2019 & 0 & 100.00 \\
\hline $\begin{array}{l}\text { Moore, G.; Wilding, H.; Gray, K.; } \\
\text { Castle, D. }\end{array}$ & $\begin{array}{l}\text { Participatory methods to engage health service } \\
\text { users in the development of electronic health } \\
\text { resources: Systematic review }\end{array}$ & J Med. Internet Res. & 4.945 & 2019 & 0 & 100.00 \\
\hline $\begin{array}{l}\text { Feijt, M.; De Kort, Y.; Bongers, I.; } \\
\text { IJsselsteijn, W. }\end{array}$ & $\begin{array}{l}\text { Perceived drivers and barriers to the adoption of } \\
\text { eMental health by psychologists: The construction } \\
\text { of the levels of adoption of eMental health model }\end{array}$ & J. Med. Internet Res. & 4.945 & 2018 & 10 & 100.00 \\
\hline $\begin{array}{l}\text { Sjöström, A.; Hörnsten, A.; } \\
\text { Hajdarevic, S.; Emmoth, A.; } \\
\text { Isaksson, U. }\end{array}$ & $\begin{array}{l}\text { Primary health care nurses' experiences of } \\
\text { consultations with internet-informed patients: } \\
\text { qualitative study }\end{array}$ & J. Med. Internet Res. & 4.945 & 2019 & 0 & 100.00 \\
\hline $\begin{array}{l}\text { Abbott-Garner, P.; Richardson, J.; } \\
\text { Jones, R. }\end{array}$ & $\begin{array}{l}\text { The impact of superfast broadband, tailored } \\
\text { booklets for households, and discussions with } \\
\text { general practitioners on personal electronic health } \\
\text { readiness: Cluster factorial quasi-randomized } \\
\text { control trial }\end{array}$ & J Med. Internet Res. & 4.945 & 2019 & 0 & 100.00 \\
\hline $\begin{array}{l}\text { Kip, H.; Kelders, S.M.; Bouman, } \\
\text { Y.H.A.; Van Gemert-Pijnen, L.J.E.W.C. }\end{array}$ & $\begin{array}{l}\text { The importance of systematically reporting and } \\
\text { reflecting on eHealth development: Participatory } \\
\text { development process of a virtual reality application } \\
\text { for forensic mental health care }\end{array}$ & J. Med. Internet Res. & 4.945 & 2019 & 0 & 100.00 \\
\hline Schofield, P.; Shaw, T.; Pascoe, M. & $\begin{array}{l}\text { Toward comprehensive patient-centric care by } \\
\text { integrating digital health technology with direct } \\
\text { clinical contact in Australia }\end{array}$ & J. Med. Internet Res. & 4.945 & 2019 & 0 & 100.00 \\
\hline $\begin{array}{l}\text { Neijenhuijs, K.I.; Van Der Hout, A.; } \\
\text { Veldhuijzen, E.; Scholten-Peeters, } \\
\text { G.G.M.; Van Uden-Kraan, C.F.; } \\
\text { Cuijpers, P.; Verdonck-De Leeuw, I.M. }\end{array}$ & $\begin{array}{l}\text { Translation of the eHealth Impact Questionnaire } \\
\text { for a population of Dutch electronic health users: } \\
\text { Validation study }\end{array}$ & J. Med. Internet Res. & 4.945 & 2019 & 0 & 100.00 \\
\hline $\begin{array}{l}\text { Gray, C.; Gravesande, J.; Hans, P.; Nie, } \\
\text { J.; Sharpe, S.; Loganathan, M.; Lyons, } \\
\text { R.; Cott, C. }\end{array}$ & $\begin{array}{l}\text { Using exploratory trials to identify relevant } \\
\text { contexts and mechanisms in complex electronic } \\
\text { health interventions: Evaluating the electronic } \\
\text { patient-reported outcome tool }\end{array}$ & J. Med. Internet Res. & 4.945 & 2019 & 0 & 100.00 \\
\hline $\begin{array}{l}\text { Ospina-Pinillos, L.; Davenport, T.; } \\
\text { Diaz, A.; Navarro-Mancilla, A.; Scott, } \\
\text { E.; Hickie, I. }\end{array}$ & $\begin{array}{l}\text { Using participatory design methodologies to } \\
\text { co-design and culturally adapt the Spanish version } \\
\text { of the mental health eClinic: Qualitative study }\end{array}$ & J. Med. Internet Res. & 4.945 & 2019 & 0 & 100.00 \\
\hline $\begin{array}{l}\text { Carroll, J.; Tobin, J.; Luque, A.; Farah, } \\
\text { S.; Sanders, M.; Cassells, A.; Fine, S.; } \\
\text { Cross, W.; Boyd, M.; Holder, T.; } \\
\text { Thomas, M.; Overa, C.; Fiscella, K. }\end{array}$ & $\begin{array}{l}\text { "Get Ready and Empowered About Treatment" } \\
\text { (GREAT) Study: A pragmatic randomized } \\
\text { controlled trial of activation in persons living } \\
\text { with HIV }\end{array}$ & J. Gen. Intern. Med. & 4.606 & 2019 & 0 & 100.00 \\
\hline
\end{tabular}


Table A1. Cont.

\begin{tabular}{|c|c|c|c|c|c|c|}
\hline Author & Title & Journal & $\begin{array}{l}\text { Impact } \\
\text { Factor }\end{array}$ & Year & Citations & InOrdinatio 10 \\
\hline $\begin{array}{c}\text { Mapar, M.; Jafari, M.; Mansouri, N.; } \\
\text { Arjmandi, R.; Azizinejad, R.; Ramos, } \\
\text { T. }\end{array}$ & $\begin{array}{l}\text { Sustainability indicators for municipalities of } \\
\text { megacities: Integrating health, safety and } \\
\text { environmental performance }\end{array}$ & Ecological Indicators & 4.49 & 2017 & 20 & 100.00 \\
\hline $\begin{array}{c}\text { Maillart, E.; Labauge, P.; Cohen, M.; } \\
\text { Maarouf, A.; Vukusic, S.; Donze, C.; } \\
\text { Gallien, P.; De Seze, J.; Bourre, B.; } \\
\text { Moreau, T.; Louapre, C.; Mayran, P.; } \\
\text { Bieuvelet, S.; Vallee, M.; Bertillot, F.; } \\
\text { Klaeyle, L.; Argoud, A.-L.; Zinai, S.; } \\
\text { Tourbah, A. }\end{array}$ & $\begin{array}{l}\text { MSCopilot, a new multiple sclerosis } \\
\text { self-assessment digital solution: Results of a } \\
\text { comparative study versus standard tests }\end{array}$ & Eur. J. Neurol. & 4.387 & 2019 & 0 & 100.00 \\
\hline $\begin{array}{c}\text { Li, D.; Brown, C.; Gallo, C.; Morgan, } \\
\text { E.; Sullivan, P.; Young, S.; Mustanski, } \\
\text { B. }\end{array}$ & $\begin{array}{l}\text { Design considerations for implementing eHealth } \\
\text { behavioral interventions for HIV prevention in } \\
\text { evolving sociotechnical landscapes }\end{array}$ & Curr. HIV/AIDS Rep. & 4.382 & 2019 & 0 & 100.00 \\
\hline $\begin{array}{l}\text { [87] Jacob, C.; Sanchez-Vazquez, A.; } \\
\text { Ivory, C. }\end{array}$ & $\begin{array}{l}\text { Clinicians' role in the adoption of an oncology } \\
\text { decision support app in Europe and its } \\
\text { implications for organizational practices: } \\
\text { Qualitative case study }\end{array}$ & JMIR $m$ Health and $u$ Health & 4.301 & 2019 & 0 & 100.00 \\
\hline $\begin{array}{l}\text { Knitza, J.; Tascilar, K.; Messner, E.-M.; } \\
\text { Meyer, M.; Vossen, D.; Pulla, A.; } \\
\text { Bosch, P.; Kittler, J.; Kleyer, A.; } \\
\text { Sewerin, P.; Mucke, J.; Haase, I., } \\
\text { Simon, D.; Krusche, M. }\end{array}$ & $\begin{array}{l}\text { German mobile apps in rheumatology: Review and } \\
\text { analysis using the Mobile Application Rating } \\
\text { Scale (MARS) }\end{array}$ & JMIR mHealth and $u$ Health & 4.301 & 2019 & 0 & 100.00 \\
\hline Mountford, $\mathrm{N}$. & $\begin{array}{l}\text { Managing by proxy: Organizational networks as } \\
\text { institutional levers in evolving public good } \\
\text { markets }\end{array}$ & J. Bus. Res. & 4.028 & 2019 & 0 & 100.00 \\
\hline [86] Yang, H.; Guo, X.; Wu, T. & $\begin{array}{l}\text { Exploring the influence of the online physician } \\
\text { service delivery process on patient satisfaction }\end{array}$ & Decision Support Systems & 3.847 & 2015 & 40 & 100.00 \\
\hline Farid, S. & $\begin{array}{l}\text { Conceptual framework of the impact of health } \\
\text { technology on healthcare system }\end{array}$ & Front Pharmacol & 3.845 & 2019 & 0 & 100.00 \\
\hline $\begin{array}{l}\text { Niemelä, R.; Pikkarainen, M.; Ervasti, } \\
\text { M.; Reponen, J. }\end{array}$ & $\begin{array}{l}\text { The change of pediatric surgery practice due to the } \\
\text { emergence of connected health technologies }\end{array}$ & Technol. Forecast. Soc. Change & 3.815 & 2019 & 0 & 100.00 \\
\hline $\begin{array}{l}\text { Canonica, G.; Bachert, C.; Hellings, P.; } \\
\text { Ryan, D.; Valovirta, E.; Wickman, M.; } \\
\text { De Beaumont, O.; Bousquet, J. }\end{array}$ & $\begin{array}{l}\text { Allergen Immunotherapy (AIT): A prototype of } \\
\text { Precision Medicine }\end{array}$ & World Allergy Organization Journal & 3.684 & 2015 & 40 & 100.00 \\
\hline $\begin{array}{l}\text { Peeters, J.M.; Krijgsman, J.W.; Brabers, } \\
\text { A.E.; De Jong, J.D.; Friele, R.D. }\end{array}$ & $\begin{array}{l}\text { Use and uptake of eHealth in general practice: A } \\
\text { cross-sectional survey and focus group study } \\
\text { among health care users and general practitioners }\end{array}$ & JMIR Medical Informatics & 3.188 & 2016 & 30 & 100.00 \\
\hline $\begin{array}{l}\text { [93] Van Der Linden, S.; Sitskoorn, M.; } \\
\text { Rutten, G.J.; Gehring, K. }\end{array}$ & $\begin{array}{l}\text { Feasibility of the evidence-based cognitive } \\
\text { telerehabilitation program Remind for patients } \\
\text { with primary brain tumors }\end{array}$ & J. Neurooncol. & 3.129 & 2018 & 10 & 100.00 \\
\hline Bervell, B.; Al-Samarraie, H. & $\begin{array}{l}\text { A comparative review of mobile health and } \\
\text { electronic health utilization in sub-Saharan } \\
\text { African countries }\end{array}$ & Soc. Sci. Med. & 3.087 & 2019 & 0 & 100.00 \\
\hline $\begin{array}{l}\text { [98] Boussada, R.; Hamdane, B.; } \\
\text { Elhdhili, M.E.; Saidane, L.A. }\end{array}$ & $\begin{array}{l}\text { Privacy-preserving aware data transmission for } \\
\text { IoT-based e-health }\end{array}$ & Computer Networks & 3.03 & 2019 & 0 & 100.00 \\
\hline Mondal, S.; Mukherjee, N. & $\begin{array}{l}\text { An efficient reachability query based pruning } \\
\text { algorithm in e-health scenario }\end{array}$ & J. Biomed. Inf. & 2.95 & 2019 & 0 & 100.00 \\
\hline Perazzo, J.; Reyes, D.; Webel, A. & $\begin{array}{l}\text { A systematic review of health literacy interventions } \\
\text { for people living with HIV }\end{array}$ & AIDS Behav. & 2.908 & 2017 & 20 & 100.00 \\
\hline $\begin{array}{l}\text { Carmel, A.; Cornelius-Schecter, A.; } \\
\text { Frankel, B.; Jannat-Khah, D.; Sinha, S.; } \\
\text { Pelzman, F; Safford, M. }\end{array}$ & $\begin{array}{l}\text { Evaluation of the Patient Activated Learning } \\
\text { System (PALS) to improve knowledge acquisition, } \\
\text { retention, and medication decision making among } \\
\text { hypertensive adults: Results of a pilot randomized } \\
\text { controlled trial }\end{array}$ & Patient Educ. Couns. & 2.821 & 2019 & 0 & 100.00 \\
\hline $\begin{array}{l}\text { Chang, Y.W.; Hsu, P.Y.; Wang, Y.; } \\
\text { Chang, P.Y. }\end{array}$ & $\begin{array}{l}\text { Integration of online and offline health services: } \\
\text { The role of doctor-patient online interaction }\end{array}$ & Patient Educ. Couns. & 2.821 & 2019 & 0 & 100.00 \\
\hline $\begin{array}{l}\text { Fang, S.Y.; Wang, Y.L.; Lu, W.H.; Lee, } \\
\text { K.T.; Kuo, Y.L.; Fetzer, S.J. }\end{array}$ & $\begin{array}{l}\text { Long-term effectiveness of an E-based survivorship } \\
\text { care plan for breast cancer survivors: A } \\
\text { quasi-experimental study }\end{array}$ & Patient Educ. Couns. & 2.821 & 2019 & 0 & 100.00 \\
\hline $\begin{array}{l}\text { Helle, C.; Hillesund, E.; Wills, A.; } \\
\text { Øverby, N. }\end{array}$ & $\begin{array}{l}\text { Examining the effects of an eHealth intervention } \\
\text { from infant age } 6 \text { to } 12 \text { months on child eating } \\
\text { behaviors and maternal feeding practices one year } \\
\text { after cessation: The Norwegian randomized } \\
\text { controlled trial Early Food for Future Health }\end{array}$ & Plos One & 2.776 & 2019 & 0 & 100.00 \\
\hline $\begin{array}{l}\text { Denis, F.; Voog, E.; Pointreau, Y.; } \\
\text { Bourgeois, H.; Seegers, V.; Le Du, K. }\end{array}$ & $\begin{array}{l}\text { Prospective study of a web-mediated management } \\
\text { of febrile neutropenia related to } \\
\text { chemotherapy (Bioconnect) }\end{array}$ & Support Care Cancer & 2.754 & 2019 & 0 & 100.00 \\
\hline $\begin{array}{l}\text { Sadegh, S.S.; Saadat, P.K.; Sepehri, } \\
\text { M.M.; Assadi, V. }\end{array}$ & $\begin{array}{l}\text { A framework for m-health service development } \\
\text { and success evaluation }\end{array}$ & $\begin{array}{l}\text { International Journal of Medical } \\
\text { Informatics }\end{array}$ & 2.721 & 2018 & 10 & 100.00 \\
\hline $\begin{array}{l}\text { Frontoni, E.; Mancini, A.; Baldi, M.; } \\
\text { Paolanti, M.; Moccia, S.; Zingaretti, P.; } \\
\text { Landro, V.;Misericordia, P. }\end{array}$ & $\begin{array}{l}\text { Sharing health data among general practitioners: } \\
\text { The Nu.Sa. project }\end{array}$ & $\begin{array}{l}\text { International Journal of Medical } \\
\text { Informatics }\end{array}$ & 2.721 & 2019 & 0 & 100.00 \\
\hline $\begin{array}{l}\text { Gu, D.; Li, T.; Wang, X.; Yang, X.; } \\
\text { Yu, Z. }\end{array}$ & $\begin{array}{l}\text { Visualizing the intellectual structure and evolution } \\
\text { of electronic health and telemedicine research }\end{array}$ & $\begin{array}{l}\text { International Journal of Medical } \\
\text { Informatics }\end{array}$ & 2.721 & 2019 & 0 & 100.00 \\
\hline $\begin{array}{l}\text { Kagawa, R.; Shinohara, E; Imai, T.; } \\
\text { Kawazoe, Y;; Ohe, K. }\end{array}$ & $\begin{array}{l}\text { Bias of inaccurate disease mentions in electronic } \\
\text { health record-based phenotyping }\end{array}$ & $\begin{array}{l}\text { International Journal of Medical } \\
\text { Informatics }\end{array}$ & 2.721 & 2019 & 0 & 100.00 \\
\hline $\begin{array}{l}\text { Parks, R.; Wigand, R.; Othmani, M.; } \\
\text { Serhier, Z;; Bouhaddou, O. }\end{array}$ & $\begin{array}{l}\text { Electronic health records implementation in } \\
\text { Morocco: Challenges of silo efforts and } \\
\text { recommendations for improvements }\end{array}$ & $\begin{array}{l}\text { International Journal of Medical } \\
\text { Informatics }\end{array}$ & 2.721 & 2019 & 0 & 100.00 \\
\hline $\begin{array}{l}\text { Krijnen-De Bruin, E.; Muntingh, A.; } \\
\text { Hoogendoorn, A.; Van Straten, A.; } \\
\text { Batelaan, N.; Marsingh, O.; Van } \\
\text { Balkom, A.; Van Meijel, B. }\end{array}$ & $\begin{array}{l}\text { The GET READY relapse prevention programme } \\
\text { for anxiety and depression: A mixed-methods } \\
\text { study protocol }\end{array}$ & BMC Psychiatry & 2.666 & 2019 & 0 & 100.00 \\
\hline
\end{tabular}


Table A1. Cont.

\begin{tabular}{|c|c|c|c|c|c|c|}
\hline Author & Title & Journal & $\begin{array}{l}\text { Impact } \\
\text { Factor }\end{array}$ & Year & Citations & InOrdinatio 10 \\
\hline Habboushe, J.; Altman, C.; Lip, G. & $\begin{array}{l}\text { Time trends in use of the CHADS } 2 \text { and CHA } 2 \text { DS } \\
2 \text { VASc scores, and the geographical and specialty } \\
\text { uptake of these scores from a popular online } \\
\text { clinical decision tool and medical reference }\end{array}$ & $\begin{array}{l}\text { International Journal of Clinical } \\
\text { Practice }\end{array}$ & 2.613 & 2019 & 0 & 100.00 \\
\hline $\begin{array}{l}\text { Droes, R.M.; Van Rijn, A.;Rus, E.; } \\
\text { Dacier, S.; Meiland, F. }\end{array}$ & $\begin{array}{l}\text { Utilization, effect, and benefit of the individualized } \\
\text { Meeting Centers Support Program for people with } \\
\text { dementia and caregivers }\end{array}$ & Clin. Interv. Aging. & 2.585 & 2019 & 0 & 100.00 \\
\hline $\begin{array}{l}\text { Berger, M.; Steinberg, D.; Askew, S.; } \\
\text { Gallis, J; Treadway, C.; Egger, J; Kay, } \\
\text { M.; Batch, B.; Finkelstein, E.; Devries, } \\
\text { A.; Brewer, A.; Bennett, G. }\end{array}$ & $\begin{array}{l}\text { The Balance protocol: A pragmatic weight gain } \\
\text { prevention randomized controlled trial for } \\
\text { medically vulnerable patients within primary care }\end{array}$ & BMC Public Health & 2.567 & 2019 & 0 & 100.00 \\
\hline Ohana, S.; Barnoy, S. & Israeli e-patients' Informational Needs & Nurs. Outlook & 2.54 & 2019 & 0 & 100.00 \\
\hline $\begin{array}{l}\text { Albarrak, A.I.; Mohammed, R.; } \\
\text { Almarshoud, N.; Almujalli, L.; } \\
\text { Aljaeed, R.; Altuwaijiri, S.; } \\
\text { Albohairy, T. }\end{array}$ & $\begin{array}{l}\text { Assessment of physician's knowledge, perception } \\
\text { and willingness of telemedicine in Riyadh region, } \\
\text { Saudi Arabia }\end{array}$ & J. Infect. Public Health & 2.487 & 2019 & 0 & 100.00 \\
\hline Zhou, J;; Fan, T. & $\begin{array}{l}\text { Understanding the factors influencing patient } \\
\text { e-health literacy in online health communities } \\
\text { (OHCs): A social cognitive theory perspective }\end{array}$ & Int. J. Environ. Res Public Health & 2.468 & 2019 & 0 & 100.00 \\
\hline Rathnayake, S.; Senevirathna, A. & $\begin{array}{l}\text { Self-reported eHealth literacy skills among nursing } \\
\text { students in Sri Lanka: A cross-sectional study }\end{array}$ & Nurse Educ Today & 2.442 & 2019 & 0 & 100.00 \\
\hline $\begin{array}{l}\text { [102] Waschkau, A.; Wilfling, D.; } \\
\text { Steinhaeuser, J. }\end{array}$ & $\begin{array}{l}\text { Are Big Data analytics helpful in caring for } \\
\text { multimorbid patients in general practice?-a } \\
\text { scoping review }\end{array}$ & BMC Fam. Pract. & 2.431 & 2019 & 0 & 100.00 \\
\hline $\begin{array}{l}\text { Firet, L.; De Bree, C.; Verhoeks, C.; } \\
\text { Teunissen, D.; Lagro-Janssen, A. }\end{array}$ & $\begin{array}{l}\text { Mixed feelings: General practitioners' attitudes } \\
\text { towards eHealth for stress urinary incontinence-a } \\
\text { qualitative study }\end{array}$ & BMC Fam. Pract. & 2.431 & 2019 & 0 & 100.00 \\
\hline $\begin{array}{c}\text { Gagnon, M.-P.;Ndiaye, M.A.; } \\
\text { Larouche, A.; Chabot, G.; Chabot, C., } \\
\text { Buyl, R.; Fortin, J-.P.; Giguere, A.; } \\
\text { Leblanc, A.; Legare, F;; Motulsky, } \\
\text { A.;Sicotte, C.; Witteman, H.O.; } \\
\text { Kavanagh, E.; Lepinay, F.; Roberge, J.; } \\
\text { Deletroz, C.; } \\
\text { Abbasgholizadeh-Rahimi, S. }\end{array}$ & $\begin{array}{l}\text { Optimising patient active role with a user-centred } \\
\text { eHealth platform (CONCERTO plus) in chronic } \\
\text { diseases management: A study protocol for a pilot } \\
\text { cluster randomised controlled trial }\end{array}$ & BMJ Open & 2.376 & 2019 & 0 & 100.00 \\
\hline $\begin{array}{l}\text { Beishuizen, C.; Akenine, U.; Barbera, } \\
\text { M.; Rosenberg, A.; Fallah Pour, M.; } \\
\text { Richard, E.; Soininen, H.; } \\
\text { Mangialasche, F; Kivipelto, M.; Pols, } \\
\text { A.; Moll Van Charante, E. }\end{array}$ & $\begin{array}{l}\text { Integrating nurses' experiences with supporting } \\
\text { behaviour change for cardiovascular prevention } \\
\text { into a self-management internet plattorm in } \\
\text { Finland and the Netherlands: A qualitative study }\end{array}$ & BMJ Open & 2.376 & 2019 & 0 & 100.00 \\
\hline $\begin{array}{l}\text { Kocher, A.; Simon, M.; Dwyer, A.A.; } \\
\text { Villiger, P.M.; Kunzler-Heule, P.; De } \\
\text { Geest, S.; Berben, L.; Nicca, D. }\end{array}$ & $\begin{array}{l}\text { Developing a rare disease chronic care model: } \\
\text { Management of systemic sclerosis (MANOSS) } \\
\text { study protocol }\end{array}$ & Journal of Advanced Nursing & 2.376 & 2019 & 0 & 100.00 \\
\hline Ladan, M.A.; Wharrad, H.; Windle, R. & $\begin{array}{l}\text { eHealth adoption and use among healthcare } \\
\text { professionals in a tertiary hospital in Sub-Saharan } \\
\text { Africa: A Qmethodology study }\end{array}$ & PEERJ & 2.353 & 2019 & 0 & 100.00 \\
\hline $\begin{array}{l}\text { Huang, R.-C.; Silva, D.; Beilin, L.; } \\
\text { Neppe, C.; Mackie, K.; Roffey, E.; } \\
\text { Gibson, L.; D'vaz, N.;Christian, } \\
\text { H.;Reid, C.; Prescott, S. }\end{array}$ & $\begin{array}{l}\text { Feasibility of conducting an early pregnancy diet } \\
\text { and lifestyle e-health intervention: The Pregnancy } \\
\text { Lifestyle Activity Nutrition (PLAN) project }\end{array}$ & J. Dev. Orig. Health Dis. & 2.34 & 2019 & 0 & 100.00 \\
\hline $\begin{array}{l}\text { Vamos, C.A.; Griner, S.B.; Kirchharr, } \\
\text { C.; Green, S.M.; Debate, R.; Daley, } \\
\text { E.M.; Quinonez, R.B.; Boggess, K.A; } \\
\text { Jacobs, T.; Christiansen, S. }\end{array}$ & $\begin{array}{l}\text { The development of a theory-based eHealth app } \\
\text { prototype to promote oral health during prenatal } \\
\text { care visits }\end{array}$ & Transl. Behav. Med. & 2.237 & 2019 & 0 & 100.00 \\
\hline $\begin{array}{l}\text { [59] Devan, H.; Godfrey, H.K.; Perry, } \\
\text { M.A.; Hempel, D.; Saipe, B.; Hale, L.; } \\
\text { Grainger, R. }\end{array}$ & $\begin{array}{l}\text { Current practices of health care providers in } \\
\text { recommending online resources for chronic pain } \\
\text { self-management }\end{array}$ & J. Pain Res. & 2.236 & 2019 & 0 & 100.00 \\
\hline $\begin{array}{l}\text { Vehko, T.; Hyppönen, H.; Puttonen, S.; } \\
\text { Kujala, S.; Ketola, E; Tuukkanen, J.; } \\
\text { Aalto, A.-M.; Heponiemi, T. }\end{array}$ & $\begin{array}{l}\text { Experienced time pressure and stress: electronic } \\
\text { health records usability and information } \\
\text { technology competence play a role }\end{array}$ & BMC Med. Inform. Decis. Mak. & 2.067 & 2019 & 0 & 100.00 \\
\hline [92] Duruturk, N.; Özköslü, M.A. & $\begin{array}{l}\text { Effect of tele-rehabilitation on glucose control, } \\
\text { exercise capacity, physical fitness, muscle strength } \\
\text { and psychosocial status in patients with type 2 } \\
\text { diabetes: A double blind randomized } \\
\text { controlled trial }\end{array}$ & Prim. Care Diabetes & 2.008 & 2019 & 0 & 100.00 \\
\hline $\begin{array}{l}\text { Van Schaik, P.; Thornhill, E.; Davies, } \\
\text { M.; Flynn, D.; Kusev, P. }\end{array}$ & $\begin{array}{l}\text { The use of information in online healthcare } \\
\text { provider choice }\end{array}$ & Int. J. Hum. Comput. Stud. & 2.006 & 2019 & 0 & 100.00 \\
\hline Arduini, D.; Zanfei, A. & $\begin{array}{l}\text { An overview of scholarly research on public } \\
\text { e-services? A meta-analysis of the literature }\end{array}$ & Telecomm Policy & 2.000 & 2014 & 50 & 100.00 \\
\hline $\begin{array}{c}\text { Doarn, C.; Zacharias, S.; Keck, C.; } \\
\text { Tabangin, M.; Dealarcon, A.; Kelchner, } \\
\text { L. }\end{array}$ & $\begin{array}{l}\text { Design and implementation of an interactive } \\
\text { website for pediatric voice therapy - the concept of } \\
\text { in-between care: A telehealth model }\end{array}$ & Telemedicine and $\mathrm{e}$-Health & 1.996 & 2019 & 0 & 100.00 \\
\hline $\begin{array}{l}\text { Doarn, C.R.; Dorogi, A.; Tikhtman, R.; } \\
\text { Pallerla, H.; Vonder Meulen, M.B. }\end{array}$ & $\begin{array}{l}\text { Opinions on the role of telehealth in a large } \\
\text { Midwest academic health center: A case study }\end{array}$ & Telemedicine and $\mathrm{e}$-Health & 1.996 & 2019 & 0 & 100.00 \\
\hline $\begin{array}{l}\text { Rebchuk, A.; Deptuck, H.; O'Neill, Z.; } \\
\text { Fawcett, D.; Silverberg, N.; Field, T. }\end{array}$ & $\begin{array}{l}\text { Validation of a novel telehealth administration } \\
\text { protocol for the NIH toolbox-cognition battery }\end{array}$ & Telemedicine and $\mathrm{e}$-Health & 1.996 & 2019 & 0 & 100.00 \\
\hline $\begin{array}{l}\text { Murchie, P.; Masthoff, J.; Walter, F.; } \\
\text { Rahman, K.; Allan, J.; Burrows, N.; } \\
\text { Proby, C.; Lee, A.; Johnston, M.; } \\
\text { Durrani, A.; Depasquale, I.; Brant, B.; } \\
\text { Neilson, A.; Meredith, F; Treweek, S.; } \\
\text { Hall, S.; McDonald, A. }\end{array}$ & $\begin{array}{l}\text { Achieving Self-Directed Integrated Cancer } \\
\text { Aftercare (ASICA) in melanoma: Protocol for a } \\
\text { randomised patient-focused pilot trial of delivering } \\
\text { the ASICA intervention as a means to earlier } \\
\text { detection of recurrent and second } \\
\text { primary melanoma }\end{array}$ & Trials & 1.975 & 2019 & 0 & 100.00 \\
\hline Gerrits, $\mathrm{O}$. & $\begin{array}{l}\text { The future of healthcare has arrived: Who dares } \\
\text { take up the challenge? }\end{array}$ & Neth. Heart J. & 1.972 & 2019 & 0 & 100.00 \\
\hline
\end{tabular}


Table A1. Cont.

\begin{tabular}{|c|c|c|c|c|c|c|}
\hline Author & Title & Journal & $\begin{array}{l}\text { Impact } \\
\text { Factor }\end{array}$ & Year & Citations & InOrdinatio 10 \\
\hline $\begin{array}{l}\text { Van Den Bosch, S.; Van De Voort, N.; } \\
\text { Xi, T.; Kool, R.; Bergé, S.; Faber, M. }\end{array}$ & $\begin{array}{l}\text { Oral and maxillofacial surgery is ready for } \\
\text { patient-centred eHealth interventions-the } \\
\text { outcomes of a scoping review }\end{array}$ & $\begin{array}{l}\text { International Journal of Oral and } \\
\text { Maxillofacial Surgery }\end{array}$ & 1.961 & 2019 & 0 & 100.00 \\
\hline Warth, L.L.; Dyb, K. & $\begin{array}{l}\text { eHealth initiatives; the relationship between project } \\
\text { work and institutional practice }\end{array}$ & BMC Health Serv, Res, & 1.932 & 2019 & 0 & 100.00 \\
\hline $\begin{array}{l}\text { Heisler, M.; Choi, H.; Mase, R.; Long, } \\
\text { J.A.; Reeves, P.J. }\end{array}$ & $\begin{array}{l}\text { Effectiveness of technologically enhanced peer } \\
\text { support in improving glycemic management } \\
\text { among predominantly African American, } \\
\text { low-income adults with diabetes }\end{array}$ & Diabetes Educator & 1.910 & 2019 & 0 & 100.00 \\
\hline $\begin{array}{l}\text { Ferguson, M.; Leighton, P.; Brandreth, } \\
\text { M.; Wharrad, H. }\end{array}$ & $\begin{array}{l}\text { Development of a multimedia educational } \\
\text { programme for first-time hearing aid users: A } \\
\text { participatory design }\end{array}$ & International Journal of Audiology & 1.821 & 2018 & 10 & 100.00 \\
\hline Leung, C.; Shaipanich, T. & $\begin{array}{l}\text { Current practice in the management of pulmonary } \\
\text { nodules detected on computed tomography } \\
\text { chest scans }\end{array}$ & Can. Respir. J. & 1.803 & 2019 & 0 & 100.00 \\
\hline Shin, E.; Shim, J-M. & $\begin{array}{l}\text { Listen to doctors, friends, or both? Embedded they } \\
\text { produce thick knowledge and promote health }\end{array}$ & J. Health Cоттии. & 1.773 & 2019 & 0 & 100.00 \\
\hline $\begin{array}{l}\text { [66] Jansson, M.M.; Harjumaa, M.; } \\
\text { Puhto, A.-P.; Pikkarainen, M. }\end{array}$ & $\begin{array}{l}\text { Healthcare professionals' proposed eHealth needs } \\
\text { in elective primary fast-track hip and knee } \\
\text { arthroplasty journey: A qualitative interview study }\end{array}$ & J. Clin. Nurs. & 1.757 & 2019 & 0 & 100.00 \\
\hline Kiberu, V.; Mars, M.; Scott, R. & $\begin{array}{l}\text { Development of an evidence-based e-health } \\
\text { readiness assessment framework for Uganda }\end{array}$ & $\begin{array}{l}\text { Health Information Management } \\
\text { Journal }\end{array}$ & 1.742 & 2019 & 0 & 100.00 \\
\hline $\begin{array}{l}\text { Aromatario, O.; Hoye, A.V.; Vuillemin, } \\
\text { A.; Foucaut, A.-M.; Crozet, C.; } \\
\text { Pommier, J; Cambon, L. }\end{array}$ & $\begin{array}{l}\text { How do mobile health applications support } \\
\text { behaviour changes? A scoping review of mobile } \\
\text { health applications relating to physical activity and } \\
\text { eating behaviours }\end{array}$ & Public Health & 1.696 & 2019 & 0 & 100.00 \\
\hline $\begin{array}{l}\text { Lie, S., Karlsen, B., Graue, M. and } \\
\text { Oftedal, B. }\end{array}$ & $\begin{array}{l}\text { The influence of an eHealth intervention for adults } \\
\text { with type } 2 \text { diabetes on the patient-nurse } \\
\text { relationship: A qualitative study }\end{array}$ & Scand. J. Caring Sci. & 1.642 & 2019 & 0 & 100.00 \\
\hline Momenipour, A.; Pennathur, P. & $\begin{array}{l}\text { Balancing documentation and direct patient care } \\
\text { activities: A study of a mature electronic health } \\
\text { record system }\end{array}$ & Int. J. Ind. Ergon. & 1.571 & 2019 & 0 & 100.00 \\
\hline $\begin{array}{l}\text { [60] Zitkus, V.; Butkiene, R.; Butleris, } \\
\text { R.; Maskeliunas, R.; Damasevicius, R.; } \\
\text { Wozniak, M. }\end{array}$ & $\begin{array}{l}\text { Minimalistic approach to coreference resolution in } \\
\text { Lithuanian medical records }\end{array}$ & Comput. Math. Methods Med. & 1.563 & 2019 & 0 & 100.00 \\
\hline $\begin{array}{l}\text { Wingo, B.C.; Yang, D.; Davis, D.; } \\
\text { Padalabalanarayanan, S.; Hopson, B.; } \\
\text { Thirumalai, M.; Rimmer, J.H. }\end{array}$ & $\begin{array}{l}\text { Lessons learned from a blended } \\
\text { telephone/e-health platform for caregivers in } \\
\text { promoting physical activity and nutrition in } \\
\text { children with a mobility disability }\end{array}$ & Disabil. Health J. & 1.471 & 2019 & 0 & 100.00 \\
\hline $\begin{array}{l}\text { [46] Vyas, K.S.; Hambrick, H.R.; } \\
\text { Shakir, A.; Morrison, S.D.; Tran, D.C.; } \\
\text { Pearson, K.; Vasconez, H.C.; Mardini, } \\
\text { S.; Gosman, A.A.; Dobke, M.; } \\
\text { Granick, M.S. }\end{array}$ & $\begin{array}{l}\text { A systematic review of the use of telemedicine in } \\
\text { plastic and reconstructive surgery } \\
\text { and dermatology }\end{array}$ & Annals of Plastic Sugery & 1.448 & 2017 & 20 & 100.00 \\
\hline Brignone, L.; Edleson, J. & $\begin{array}{l}\text { The dating and domestic violence app Rubric: } \\
\text { Synthesizing clinical best practices and digital } \\
\text { health app standards for relationship violence } \\
\text { prevention smartphone apps }\end{array}$ & Int. J. Hum. Comput. Interact. & 1.354 & 2019 & 0 & 100.00 \\
\hline $\begin{array}{l}\text { [88] Triplett, K.; El-Behadli, A.; } \\
\text { Masood, S.; Sullivan, S.; Desai, D. }\end{array}$ & $\begin{array}{c}\text { Digital medicine program with pediatric solid } \\
\text { organ transplant patients: Perceived benefits } \\
\text { and challenges } \\
\end{array}$ & Pediatr. Transplant. & 1.326 & 2019 & 0 & 100.00 \\
\hline Garai, A.; Pentek, I.; Adamko, A. & $\begin{array}{l}\text { Revolutionizing healthcare with IoT and cognitive, } \\
\text { cloud-based telemedicine }\end{array}$ & Acta Polytechnica Hungarica & 1.286 & 2019 & 0 & 100.00 \\
\hline $\begin{array}{l}\text { Eden, R.; Burton-Jones, A.; Grant, J.; } \\
\text { Collins, R.; Staib, A; Sullivan, C. }\end{array}$ & $\begin{array}{l}\text { Digitising an Australian university hospital: } \\
\text { Qualitative analysis of staff-reported impacts }\end{array}$ & Australian Health Review & 1.228 & 2019 & 0 & 100.00 \\
\hline Ross, P.; Cross, R. & $\begin{array}{l}\text { Rise of the e-nurse: The power of social media } \\
\text { in nursing }\end{array}$ & Contemp. Nurse & 1.216 & 2019 & 0 & 100.00 \\
\hline $\begin{array}{l}\text { Castaneda, P.; Sales, A., Osborne, N. } \\
\text { and Corriere, M. }\end{array}$ & $\begin{array}{l}\text { Scope, themes, and medical accuracy of eHealth } \\
\text { peripheral artery disease community forums }\end{array}$ & Annals of Vascular Surgery & 1.179 & 2019 & 0 & 100.00 \\
\hline Charnock, V. & Electronic healthcare records and data quality & Health Info. Libr. J. & 1.179 & 2019 & 0 & 100.00 \\
\hline Peterson, S.; Kuntz, C.; Roush, J. & $\begin{array}{l}\text { Use of a modified treatment-based classification } \\
\text { system for subgrouping patients with low back } \\
\text { pain: Agreement between telerehabilitation and } \\
\text { face-to-face assessments }\end{array}$ & Physiother. Theory Pract. & 1.158 & 2019 & 0 & 100.00 \\
\hline $\begin{array}{l}\text { Veldhuijzen, G.; Van Esch, A.; } \\
\text { Klemt-Kropp, M.; Terhaar Sive Droste, } \\
\text { J; Drenth, J. }\end{array}$ & $\begin{array}{l}\text { E-patient counseling trial (E-PACO): Computer } \\
\text { based education versus nurse counseling for } \\
\text { patients to prepare for colonoscopy }\end{array}$ & $\begin{array}{l}\text { JOVE-Journal of Visualized } \\
\text { Experiments }\end{array}$ & 1.108 & 2019 & 0 & 100.00 \\
\hline $\begin{array}{l}\text { Kerdjidj, O.; Amira, A.; Ghanem, K.; } \\
\text { Ramzan, N.; Katsigiannis, S.; } \\
\text { Chouireb, F. }\end{array}$ & $\begin{array}{l}\text { An FPGA implementation of the matching pursuit } \\
\text { algorithm for a compressed sensing enabled } \\
\text { e-health monitoring platform }\end{array}$ & Microprocessors and Microsystems & 1.045 & 2019 & 0 & 100.00 \\
\hline $\begin{array}{l}\text { Serafica, R.; Inouye, J.; Lukkahatai, N.; } \\
\text { Braginsky, N.; Pacheco, M.; Daub, K. }\end{array}$ & $\begin{array}{l}\text { The use of mobile health to assist self-management } \\
\text { and access to services in a rural community }\end{array}$ & $\begin{array}{l}\text { CIN-Computers Informatics } \\
\text { Nursing }\end{array}$ & 1.029 & 2019 & 0 & 100.00 \\
\hline Abaza, H.; Marschollek, M. & $\begin{array}{l}\text { mHealth application areas and technology } \\
\text { combinations*. A comparison of literature from } \\
\text { high and low/middle income countries }\end{array}$ & Methods Inf. Med. & 1.024 & 2017 & 20 & 100.00 \\
\hline $\begin{array}{l}\text { Souza, T.; Lobão, W.; Santos, C.; } \\
\text { Almeida, M.; Moreira Júnior, E. }\end{array}$ & $\begin{array}{l}\text { Factors associated with the acceptance of the } \\
\text { influenza vaccine among health workers: } \\
\text { Knowledge, attitude and practice [Fatores } \\
\text { associados à aceitação da vacinan influenza entre } \\
\text { trabalhadores de saúde: conhecimento, } \\
\text { atitude e prática] }\end{array}$ & Cien. Saude. Colet. & 1.008 & 2019 & 0 & 100.00 \\
\hline Brown, L.J.; Jones, G.M.; Bond, M.J. & $\begin{array}{l}\text { E-health: Psychosocial challenges for South } \\
\text { Australian rural mental health consumers }\end{array}$ & Rural Remote Health & 0.985 & 2019 & 0 & 100.00 \\
\hline
\end{tabular}


Table A1. Cont.

\begin{tabular}{|c|c|c|c|c|c|c|}
\hline Author & Title & Journal & $\begin{array}{l}\text { Impact } \\
\text { Factor }\end{array}$ & Year & Citations & InOrdinatio 10 \\
\hline $\begin{array}{l}\text { Kips, J.; Lambert, J.; Ongenae, K; De } \\
\text { Sutter, A.; Verhaeghe, E. }\end{array}$ & Teledermatology in Belgium: A pilot study & Acta Clinica Belgica & 0.960 & 2019 & 0 & 100.00 \\
\hline Clanchy, K.; Tweedy, S.; Trost, S. & $\begin{array}{l}\text { The Adapted Physical Activity Program: A } \\
\text { theory-driven, evidence-based physical activity } \\
\text { intervention for people with brain impairment }\end{array}$ & Brain Impairment & 0.958 & 2019 & 0 & 100.00 \\
\hline Berndt, R.-D.; Preik, P.; Takenga, C. & $\begin{array}{l}\text { TeleDermatology: the teledermatological solution } \\
\text { mSkin }{ }^{\circledR} \text { for daily practice }\end{array}$ & Hautarzt & 0.828 & 2019 & 0 & 100.00 \\
\hline Jedamzik, S. & $\begin{array}{l}\text { Digital health and nursing: the future is now } \\
\text { [Digitale Gesundheit und Pflege: Die Zukunft } \\
\text { ist jetzt] }\end{array}$ & Unfallchirurg & 0.716 & 2019 & 0 & 100.00 \\
\hline $\begin{array}{l}\text { Khan, I.; Xitong, G.; Ahmad, Z.; } \\
\text { Shahzad, F. }\end{array}$ & $\begin{array}{l}\text { Investigating factors impelling the adoption of } \\
\text { e-health: A perspective of African expats in China }\end{array}$ & Sage Open & 0.675 & 2019 & 0 & 100.00 \\
\hline Alghazo, J.M. & $\begin{array}{l}\text { Intelligent security and privacy of electronic health } \\
\text { records using biometric images }\end{array}$ & Current Medical Imaging Reviews & 0.533 & 2019 & 0 & 100.00 \\
\hline De Leon-Castaneda, C. & $\begin{array}{l}\text { Electronic health (e-health): A conceptual } \\
\text { framework for implementation in health services }\end{array}$ & Gac. Med. Mex. & 0.283 & 2019 & 0 & 100.00 \\
\hline $\begin{array}{l}\text { Chadi, N.; Weisbaum, E.; } \\
\text { Malboeuf-Hurtubise, C.; Kohut, S.A.; } \\
\text { Viner, C.; Palaniyar, N.; Kaufman, M.; } \\
\text { Locke, J.; Vo, D.X. }\end{array}$ & $\begin{array}{l}\text { In-person vs. eHealth mindfulness-based } \\
\text { intervention for adolescents with chronic illnesses: } \\
\text { A pilot randomized trial }\end{array}$ & Adolescent Psychiatry & 0 & 2019 & 0 & 100.00 \\
\hline $\begin{array}{l}\text { Voutsidou, S.; Moraitis, E.; } \\
\text { Jelastopoulou, E.; Sissouras, A.; } \\
\text { Charalampous, G. }\end{array}$ & $\begin{array}{l}\text { Electronic health applications in primary medical } \\
\text { health care: Advantages and expectations }\end{array}$ & Archives of Hellenic Medicine & 0 & 2019 & 0 & 100.00 \\
\hline $\begin{array}{l}\text { Spyridaki, A.; Antonakos, I.; } \\
\text { Apostolakis, I.; Tountas, I. }\end{array}$ & $\begin{array}{l}\text { Investigation of the effectiveness of mobile health } \\
\text { applications for chronic diseases }\end{array}$ & Archives of Hellenic Medicine & 0 & 2019 & 0 & 100.00 \\
\hline $\begin{array}{l}\text { Ramos, A.C.; Bouzas-Lorenzo, R.; del } \\
\text { Olmo, A.M.; Buceta, B.B. }\end{array}$ & $\begin{array}{l}\text { Opinión de los facultativos y usuarios sobre } \\
\text { avances de la e-salud en atención primaria }\end{array}$ & Aten. Prim. & 0 & 2019 & 0 & 100.00 \\
\hline Guarda, $P$. & $\begin{array}{l}\text { “OK Google, am I sick?": Artificial intelligence, } \\
\text { e-health, and data protection regulation }\end{array}$ & Biolaw Journal-Rivista Di Biodiritto & 0 & 2019 & 0 & 100.00 \\
\hline $\begin{array}{l}\text { Klocek, A.; Smahelova, M.; Knapova, } \\
\text { L.; Elavsky, S. }\end{array}$ & $\begin{array}{l}\text { GPs' perspectives on eHealth use in the Czech } \\
\text { Republic: A cross-sectional mixed-design } \\
\text { survey study }\end{array}$ & BJGP Open & 0 & 2019 & 0 & 100.00 \\
\hline $\begin{array}{l}\text { Reddeman, L.; Bourgeois, N.; Angl, } \\
\text { E.N.; Heinrich, M.; Hillier, L.; Finn, H.; } \\
\text { Bosiak, B.; Agarwal, P.; Mawson, R.; } \\
\text { Propp, R.; Ivers, N.M. }\end{array}$ & $\begin{array}{l}\text { How should family physicians provide physical } \\
\text { activity advice? Qualitative study to inform the } \\
\text { design of an e-health intervention }\end{array}$ & Can. Fam. Physician & 0 & 2019 & 0 & 100.00 \\
\hline $\begin{array}{l}\text { Borries, T.M.; Dunbar, A.; Bhukhen, } \\
\text { A.; Rismany, J; Kilham, J., Feinn, R.; } \\
\text { Meehan Sr., T.P. }\end{array}$ & $\begin{array}{l}\text { The impact of telemedicine on patient } \\
\text { self-management processes and clinical outcomes } \\
\text { for patients with types I or II diabetes mellitus in } \\
\text { the United States: A scoping review }\end{array}$ & $\begin{array}{l}\text { Diabetes \& Metabolic } \\
\text { Syndrome-Clinical Research \& } \\
\text { Reviews }\end{array}$ & 0 & 2019 & 0 & 100.00 \\
\hline $\begin{array}{l}\text { Howarth, A.; Quesada, J.; Donnelly, T.; } \\
\text { Mills, P.R. }\end{array}$ & $\begin{array}{l}\text { The development of "Make One Small Change": } \\
\text { An e-health intervention for the workplace } \\
\text { developed using the Person-Based Approach }\end{array}$ & Digital Health & 0 & 2019 & 0 & 100.00 \\
\hline $\begin{array}{l}\text { Krivenko V, N.; Elishev, V.G.; } \\
\text { Kriventsova, L.A. }\end{array}$ & $\begin{array}{l}\text { The impact of innovation on the performance of } \\
\text { health care in the economic security system of } \\
\text { the region }\end{array}$ & $\begin{array}{l}\text { Ekonomika Regiona-Economy of } \\
\text { Region }\end{array}$ & 0 & 2019 & 0 & 100.00 \\
\hline $\begin{array}{l}\text { Seckin, G.; Hughes, S.; Yeatts, D.; } \\
\text { Degreve, T. }\end{array}$ & $\begin{array}{l}\text { Digital pathways to positive health perceptions: } \\
\text { Does age moderate the relationship between } \\
\text { medical satisfaction and positive health } \\
\text { perceptions among middle-aged and older } \\
\text { internet users? }\end{array}$ & Innov. Aging & 0 & 2019 & 0 & 100.00 \\
\hline Al-Sharekh, S.I.; Al-Shqeerat, K.H.A. & $\begin{array}{l}\text { Security challenges and limitations in } \\
\text { IoT environments }\end{array}$ & IJCSNS & 0 & 2019 & 0 & 100.00 \\
\hline $\begin{array}{l}\text { Azcarraga, J.; Raduban, J.; Christine } \\
\text { Gendrano, M.; Azcarraga, A. }\end{array}$ & $\begin{array}{l}\text { Identity concealment when uploading pictures of } \\
\text { patients in a tele-medicine system }\end{array}$ & $\begin{array}{l}\text { International Journal of E-Health and } \\
\text { Medical Communications }\end{array}$ & 0 & 2019 & 0 & 100.00 \\
\hline $\begin{array}{l}\text { Khennou, F.; Chaoui, N.E.H.; } \\
\text { Khamlichi, Y.I. }\end{array}$ & $\begin{array}{l}\text { A migration methodology from legacy to new } \\
\text { electronic health record based OpenEHR }\end{array}$ & $\begin{array}{l}\text { International Journal of E-Health and } \\
\text { Medical Communications }\end{array}$ & 0 & 2019 & 0 & 100.00 \\
\hline $\begin{array}{l}\text { Christie, H.L.; Martin, J.L.; Connor, J.; } \\
\text { Tange, H.J.; Verhey, F.R., de; Vugt, } \\
\text { M.E.; Orrell, M. }\end{array}$ & $\begin{array}{l}\text { eHealth interventions to support caregivers of } \\
\text { people with dementia may be proven effective, but } \\
\text { are they implementation-ready? }\end{array}$ & Internet Interv. & 0 & 2019 & 0 & 100.00 \\
\hline $\begin{array}{l}\text { De Cicco, L.; Mascolo, S.; Palmisano, } \\
\text { V.; Ribezzo, G. }\end{array}$ & $\begin{array}{l}\text { Reducing the network bandwidth requirements for } \\
360 \text { degrees immersive video streaming }\end{array}$ & Internet Technology Letters & 0 & 2019 & 0 & 100.00 \\
\hline $\begin{array}{l}\text { [78] Schimmer, R.; Orre, C.; Oberg, U.; } \\
\text { Danielsson, K.; Hornsten, A. }\end{array}$ & $\begin{array}{l}\text { Digital person-centered self-management support } \\
\text { for people with type } 2 \text { diabetes: Qualitative study } \\
\text { exploring design challenges }\end{array}$ & JMIR Diabetes & 0 & 2019 & 0 & 100.00 \\
\hline $\begin{array}{l}\text { Goodall, G.; Ciobanu, I.; Taraldsen, K.; } \\
\text { Sorgaard, J.; Marin, A., Draghici, R.; } \\
\text { Zamfir, M.-V.; Berteanu, M.; Maetzler, } \\
\text { W.; Serrano, J.A. }\end{array}$ & $\begin{array}{l}\text { The use of virtual and immersive technology in } \\
\text { creating personalized multisensory spaces for } \\
\text { people living with dementia (SENSE-GARDEN): } \\
\text { Protocol for a multisite before-after trial }\end{array}$ & JMIR Res. Protoc. & 0 & 2019 & 0 & 100.00 \\
\hline $\begin{array}{l}\text { Prasad, M.; Manjunath, C.; Murthy, } \\
\text { A.K.; Sampath, A.; Jaiswal, S.; } \\
\text { Mohapatra, A. }\end{array}$ & $\begin{array}{l}\text { Integration of oral health into primary health care: } \\
\text { A systematic review }\end{array}$ & J. Family Med. Prim. Care & 0 & 2019 & 0 & 100.00 \\
\hline Bhatia, R.; Taneja, U. & $\begin{array}{l}\text { Factors affecting indian consumers' intention to } \\
\text { use eHealth services }\end{array}$ & Journal of Health Management & 0 & 2019 & 0 & 100.00 \\
\hline Farzana, S.; Islam, S. & $\begin{array}{l}\text { Symmetric key-based patient controlled secured } \\
\text { electronic health record management protocol }\end{array}$ & Journal of High Speed Networks & 0 & 2019 & 0 & 100.00 \\
\hline Øvrelid, E; Halvorsen, M. & $\begin{array}{l}\text { Supporting process innovation with lightweight IT } \\
\text { at an emergency unit }\end{array}$ & $\begin{array}{l}\text { Journal of Integrated Design and } \\
\text { Process Science }\end{array}$ & 0 & 2019 & 0 & 100.00 \\
\hline Eke, E.; Kisi, M.; Ugurluoglu, D. & A research on awareness of e-health practices & $\begin{array}{l}\text { Journal of Mehmet Akif Ersoy } \\
\text { University Economics and } \\
\text { Administrative Sciences Faculty }\end{array}$ & 0 & 2019 & 0 & 100.00 \\
\hline
\end{tabular}


Table A1. Cont.

\begin{tabular}{|c|c|c|c|c|c|c|}
\hline Author & Title & Journal & $\begin{array}{l}\text { Impact } \\
\text { Factor }\end{array}$ & Year & Citations & InOrdinatio 10 \\
\hline Anya, O.; Tawfik, H.; Alani, M.; Hu, J. & $\begin{array}{l}\text { Cybersecurity design considerations for } \\
\text { cross-boundary clinical decision support }\end{array}$ & J Reliab. Intell. Environ. & 0 & 2019 & 0 & 100.00 \\
\hline Kruczek, A. & $\begin{array}{l}\text { e-health-modern technologies in mental } \\
\text { health care }\end{array}$ & Postepy. Psychiatrii. I. Neurologii. & 0 & 2019 & 0 & 100.00 \\
\hline $\begin{array}{l}\text { Costa, W.L.G.; Pereira, E.; } \\
\text { Kontonatsios, G. }\end{array}$ & $\begin{array}{l}\text { Use of smartphones for hypoglycaemia } \\
\text { prediction-A feasibility study }\end{array}$ & Procedia Comput Sci & 0 & 2019 & 0 & 100.00 \\
\hline Senges, E.; Guiot, D.; Chandon, J.-L. & $\begin{array}{l}\text { Desired ageing well: Predictive validity for } \\
\text { consumers aged } 50-80\end{array}$ & $\begin{array}{l}\text { Recherche Et Applications En } \\
\text { Marketing-English Edition }\end{array}$ & 0 & 2019 & 0 & 100.00 \\
\hline $\begin{array}{l}\text { Webers, C.; Beckers, E.; Boonen, A.; } \\
\text { Van Eijk-Hustings, Y; Vonkeman, H.; } \\
\text { Van De Laar, M.; Van Tubergen, A. }\end{array}$ & $\begin{array}{l}\text { Development, usability and acceptability of an } \\
\text { integrated eHealth system for spondyloarthritis in } \\
\text { the Netherlands (SpA-Net) }\end{array}$ & RMD Open & 0 & 2019 & 0 & 100.00 \\
\hline $\begin{array}{l}\text { Vesnic-Alujevic, L.; Pereira, A.G.; } \\
\text { Breitegger, M. }\end{array}$ & $\begin{array}{l}\text { Wearable sensors exploring EU policy narratives by } \\
\text { engaging the extended peer community }\end{array}$ & Tecnoscienza & 0 & 2019 & 0 & 100.00 \\
\hline $\begin{array}{l}\text { Metz, M.J.; Veerbeek, M.A.; Elfeddali, } \\
\text { I.; De Beurs, E.; Van Der } \\
\text { Feltz-Cornelis, C.M.; Beekman, A.T.F. }\end{array}$ & $\begin{array}{c}\text { Shared decision making in mental health care; } \\
\text { evaluation of the added value for patients and } \\
\text { clinicians }\end{array}$ & Tijdschr. Psychiatr. & 0 & 2019 & 0 & 100.00 \\
\hline $\begin{array}{l}\text { Allner, R.; Wilfling, D.; Kidholm, K.; } \\
\text { Steinhäuser, J. }\end{array}$ & $\begin{array}{l}\text { Telemedizinprojekte im ländlichen Raum } \\
\text { Deutschlands. Eine systematische Bewertung mit } \\
\text { dem "Modell zur Evaluation von } \\
\text { telemedizinischen Anwendungen". } \\
\text { TT-[Telemedicine projects in rural areas of } \\
\text { Germany. A systematic evaluation with the "M" }\end{array}$ & Z. Evid. Fortbild. Qual. Gesundhwes & 0 & 2019 & 0 & 100.00 \\
\hline Scherer, M.; Szecsenyi, J.; Gerlach, F. & $\begin{array}{l}\text { Digitalisation in medicine-Who proceeds and } \\
\text { who looks behind a plea for a DEGAM digital } \\
\text { strategy [Digitalisierung in der medizin -Wer } \\
\text { schreitet voran, wer schaut hinterher? Ein plädoyer } \\
\text { für eine DEGAM-digitalstrategie] }\end{array}$ & Z. Allgemeinmed. & 0 & 2019 & 0 & 100.00 \\
\hline Gebre-Mariam, M.; Bygstad, B. & $\begin{array}{l}\text { What can enterprise architecture do for healthcare? } \\
\text { A framework of antecedents and benefits }\end{array}$ & Electronic Government & 0 & 2019 & 0 & 100.00 \\
\hline $\begin{array}{l}\text { Ivanova, O.; Wambua, S.; Mwaisaka, } \\
\text { J.; Bossier, T.; Thiongo, M.; Michielsen, } \\
\text { K.; Gichangi, P. }\end{array}$ & $\begin{array}{l}\text { Evaluation of the ELIMIKA Pilot Project: } \\
\text { Improving ART adherence among HIV positive } \\
\text { youth using an eHealth intervention in } \\
\text { Mombasa, Kenya }\end{array}$ & $\begin{array}{l}\text { African Journal of Reproductive } \\
\text { Health }\end{array}$ & 0.547 & 2019 & 0 & 100.00 \\
\hline
\end{tabular}

\section{References}

1. Dhingra, D.; Dabas, A. Global strategy on digital health. Indian Pediatr. 2020, 27, 356-358. [CrossRef]

2. Negash, S.; Musa, P.; Vogel, D.; Sahay, S. Healthcare information technology for development: Improvements in people's lives through innovations in the uses of technologies. Inf. Technol. Dev. 2018, 24, 189-197. [CrossRef]

3. Ross, J.; Stevenson, F.; Lau, R.; Murray, E. Factors that influence the implementation of e-health: A systematic review of systematic reviews (an update). Implementat. Sci. 2016, 11, 146. [CrossRef]

4. Mauco, K.L.; Scott, R.E.; Mars, M. Validation of an e-health readiness assessment framework for developing countries. BMC Health Serv. Res. 2020, 20, 575. [CrossRef]

5. Ćwiklicki, M.; Schiavone, F.; Klich, J.; Pilch, K. Antecedents of use of e-health services in Central Eastern Europe: A qualitative comparative analysis. BMC Health Serv. Res. 2020, 20, 171. [CrossRef]

6. Van Houwelingen, C.; Moerman, A.; Ettema, R.; Kort, H.; Cate, O.T. Competencies required for nursing telehealth activities: A Delphi-study. Nurse Educ. Today 2016, 39, 50-62. [CrossRef]

7. Jhamb, M.; Cavanaugh, K.L.; Bian, A.; Chen, G.; Ikizler, T.A.; Unruh, M.L.; Abdel-Kader, K. Disparities in electronic health record patient portal use in nephrology clinics. Clin. J. Am. Soc. Nephrol. 2015, 10, 2013-2022. [CrossRef] [PubMed]

8. De Grood, C.; Raissi, A.; Kwon, Y.; Santana, M. Adoption of e-health technolo-gy by physicians: A scoping review. J. Multidiscip. Healthc. 2016, 9, 335-344. [CrossRef] [PubMed]

9. Wolff-Piggott, B.; Coleman, J.; Rivett, U. The clinic-level perspective on mHealth implementation: A South African case study. Inf. Technol. Dev. 2018, 24, 532-553. [CrossRef]

10. Swinkels, I.C.S.; Huygens, M.W.J.; Schoenmakers, T.M.; Nijeweme-D’hollosy, W.O.; Velsen, L.V.; Vermeulen, J.; Schoone-Harmsen, M.; Jansen, Y.J.F.M.; Schayck, O.C.P.V.; Friele, R.; et al. Lessons learned from a living lab on the broad adoption of ehealth in primary health care. J. Med. Internet Res. 2018, 20, e9110. [CrossRef] [PubMed]

11. D'Agostino, M. Electronic health strategies in The Americas: Current situation and perspectives. Rev. Peru. Med. Exp. Salud Públ. 2015, 32, 352-355. [CrossRef]

12. Kho, J.; Gillespie, N.; Martin-Khan, M. A systematic scoping review of change management practices used for telemedicine service implementations. BMC Health Serv. Res. 2020, 20, 815. [CrossRef] [PubMed]

13. Pan American Health Organization-PAHO; World Health Organization-WHO. What Is eHealtH? Available online: https: / / www.paho.org/ict4health/index.php?option=com_docman\&view=download\&alias=18-fact-sheet-paho-ehealthstrategy-8\&category_slug=materiales-comunicacion-8\&Itemid=320\&lang=en (accessed on 1 April 2020).

14. Ali, R.; Qadri, Y.A.; Zikria, Y.B.; Umer, T.; Kim, B.-S.; Kimali, S.W. Q-learning-enabled channel access in next-generation dense wireless networks for IoT-based eHealth systems. EURASIP J. Wirel. Commun. Net. 2019, 178, 1-12. [CrossRef]

15. Costa, H.J.M.; Costa, C.A.; Righi, R.R.; Antunes, R.S. Fog computing in health: A systematic literature review. Health Technol. 2020, 10, 1025-1044. [CrossRef] 
16. Schreiweis, B.; Pobiruchin, M.; Strotbaum, V.; Suleder, J.; Wiesner, M.; Bergh, B. Barriers and facilitators to the implementation of ehealth services: Systematic literature analysis. J. Med. Internet Res. 2019, 21, e14197. [CrossRef]

17. Hermes, S.; Riasanow, T.; Clemons, E.K.; Böhm, M.; Krcmar, H. The digital transformation of the healthcare industry: Exploring the rise of emerging platform ecosystems and their influence on the role of patients. Bus. Res. 2020, 13, 1033-1069. [CrossRef]

18. Parikh, S.V.; Huniewicz, P. E-health: An overview of the uses of the Internet, social media, apps, and websites for mood disorders. Curr. Opin. Psychiatry 2015, 28, 13-17. [CrossRef]

19. Pagliari, C.; Sloan, D.; Gregor, P.; Sullivan, F.; Detmer, D.; Kahan, J.P.; Oortwijn, W.; MacGillivray, S. What Is eHealth (4): A scoping exercise to map the field. J. Med. Internet Res. 2005, 7, e391. [CrossRef]

20. Srivastava, S.; Pant, M.; Abraham, A.; Agrawal, N. The technological growth in ehealth services. Comput. Mat. Methods Med. 2015, 2015, 894171. [CrossRef]

21. Fanta, G.B.; Pretorius, L.; Erasmus, L. Economic analysis of sustainable ehealth implementation in developing countries: A systematic review. Int. Assoc. Manag. Technol. 2018, 2018, 1-16.

22. Pagani, R.N.; Kovaleski, J.L.; Resende, L.M. Methodi Ordinatio: A proposed methodology to select and rank relevant scientific papers encompassing the impact factor, number of citations, and year of publication. Scientometrics 2015, 105, $2109-2135$. [CrossRef]

23. Pagani, R.N.; Kovaleski, J.L.; de Resende, L.M.M. Avanços na composição da Methodi Ordinatio para revisão sistemática de literatura. Ciência da Informação 2018, 46, 161-187.

24. De Campos, E.A.R.; Pagani, R.N.; Resende, L.M.; Pontes, J. Construction and qualitative assessment of a bibliographic portfolio using the methodology Methodi Ordinatio. Scientometrics 2018, 116, 815-842. [CrossRef]

25. Borges de Souza, A.; Fornazier, A.; Delgrossi, M.E. Local food systems: Potential for new market connections for family farming. Amb. Soc. 2020, 23, 1-20.

26. De Carvalho, G.D.G.; Sokulski, C.C.; da Silva, W.V.; de Carvalho, H.G.; de Moura, R.V.; de Francisco, A.C.; da Veiga, C.P. Bibliometrics and systematic reviews: A comparison between the Proknow-C and the Methodi Ordinatio. J. Informetr. 2020, 14, 101043. [CrossRef]

27. Research Platforms. Available online: https:/ /www.elsevier.com/pt-br/research-platforms (accessed on 2 May 2020).

28. Web of Science: Confident Research Begins Here. Available online: https://clarivate.com/webofsciencegroup/solutions/web-ofscience/ (accessed on 2 May 2020).

29. Diniz, E. Editorial. RAE-Rev. Adm. Empres. 2013, 53, 223. [CrossRef]

30. Mhealth-Market Estimates \& Trend Analysis from 2016 to 2027. Available online: https://www.grandviewresearch.com/ industry-analysis / mhealth-market (accessed on 19 September 2020).

31. Haux, R.; Ammenwerth, E.; Koch, S.; Lehmann, C.U.; Park, H.-A.; Saranto, K.; Wong, C.P. A brief survey on six basic and reduced ehealth indicators in seven countries in 2017. Appl. Clin. Inform. 2018, 9, 704-713. [CrossRef]

32. Kokol, P.; Saranto, K.; Vošner, H.B. eHealth and health informatics competences: A systemic analysis of literature production based on bibliometrics. Kybernetes 2018, 47, 1018-1030. [CrossRef]

33. ITU-Telecommunication Development Sector. Implementing e-Health in Developing Countries. Available online: https:/ / www.itu.int/ITU-D/cyb/app/docs/e-Health_prefinal_15092008.PDF (accessed on 20 September 2020).

34. Programa das Nações Unidas para o Desenvolvimento-PNUD. O que é o IDH. Available online: https: / / www.br.undp.org/ content/brazil/pt/home/idh0/conceitos/o-que-e-o-idh.html (accessed on 6 August 2021).

35. Villumsen, S.; Adler-Milstein, J.; Nøhr, C. National monitoring and evaluation of eHealth: A scoping review. JAMIA Open 2020, 3 , 132-140. [CrossRef]

36. Celes, R.S.; Rossi, T.R.A.; Barros, S.G.; Santos, C.M.L.; Cardoso, C. Telehealth as state response strategy: Systematic review. Rev. Panam. Salud Publ. 2018, 42, e84.

37. Brinkel, J.; Krämer, A.; Krumkamp, R.; Jürgen, M.; Fobil, J. Mobile phone-based mHealth approaches for public health surveillance in sub-Saharan Africa: A systematic review. Int. J. Environ. Res. Public Health 2014, 11, 11559-11582. [CrossRef]

38. Silva, B.M.C.; Rodrigues, J.J.P.C.; Torre-Díez, I.D.L.; López-Coronado, M.; Saleem, K. Mobile health: A review of current state in 2015: A review of current state in 2015. J. Biomed. Inf. 2015, 56, 265-272. [CrossRef]

39. Whitehead, L.; Seaton, P. The effectiveness of self-management mobile phone and tablet apps in long-term condition management: A systematic review. J. Med. Internet Res. 2016, 18, e4883. [CrossRef] [PubMed]

40. Baumel, A.; Faber, K.; Mathur, N.; Kane, J.; Muench, F. Enlight: A comprehensive quality and therapeutic potential evaluation tool for mobile and web-based eHealth interventions. J. Med. Internet Res. 2017, 19, e82. [CrossRef] [PubMed]

41. Litchman, M.; Rothwell, E.; Edelman, L. The diabetes online community: Older adults supporting self-care through peer health. Patient Educ. Couns. 2018, 101, 518-523. [CrossRef] [PubMed]

42. Garcia, L.; Tomas, J.; Parra, L.; Loret, J. An m-health application for cerebral stroke detection and monitoring using cloud services. Inf Int. J. Manag. 2019, 45, 319-327. [CrossRef]

43. Cruz, J.; Brooks, D.; Marques, A. Home telemonitoring in COPD: A systematic review of methodologies and patients' adherence. Int. J. Med. Inform. 2014, 83, 259-263. [CrossRef]

44. Hilty, D.M.; Crawford, A.; Teshima, J.; Chan, S.; Sunderji, N.; Yellowless, P.M.; Kramer, G.; O’Neill, P.; Fore, C.; Luo, J.; et al. A framework for telepsychiatric training and ehealth: Competency-based education, evaluation, and implications. Int. Rev. Psychiatry 2015, 27, 569-592. [CrossRef] 
45. Wentzel, J.; Vaart, R.V.D.; Bohlmeijer, E.T.; Gemert-Pijnen, J.E.W.C.V. Mixing online and face-to-face therapy: How to benefit from blended care in mental health care. JMIR Ment. Health 2016, 3, e9. [CrossRef]

46. Vyas, K.S.; Hambrick, H.R.; Shakir, A.; Morrison, S.D.; Tran, D.C.; Pearson, K.; Vasconez, H.C.; Mardini, S.; Gosman, A.A.; Dobke, M.; et al. A systematic review of the use of telemedicine in plastic and reconstructive surgery and dermatology. Ann. Plast. Surg. 2017, 78, 736-768. [CrossRef]

47. Irving, M.; Stewart, R.; Spallek, H.; Blinkhorn, A. Using teledentistry in clinical practice, an enabler to improve access to oral health care: A qualitative systematic review. J. Telemed. Telecare 2018, 24, 129-146. [CrossRef] [PubMed]

48. Schettini, P.; Shah, K.; O’Leary, C.; Patel, M.; Anderson, J.; Cho, A.; Long, A.; Bosworth, H.; Cameron, C. Keeping care connected: E-Consultation program improves access to nephrology care. J. Telemed. Telecare 2019, 25, 142-150. [CrossRef] [PubMed]

49. Pearce, C.; Bainbridge, M. A personally controlled electronic health record for Australia. J. Am. Med. Inf. Assoc. 2014, 21, 707-713. [CrossRef]

50. Bauermeister, J.; Pingel, E.; Jadwin-Cakmak, L.; Harper, G.; Horvath, K.; Weiss, G.; Dittus, P. Acceptability and preliminary efficacy of a tailored online HIV/STI testing intervention for young men who have sex with men: The get connected! program. AIDS Behav. 2015, 19, 1860-1874. [CrossRef]

51. Li, X.; Niu, J.; Karuppiah, M.; Kumari, S.; Wu, F. Secure and efficient two-factor user authentication scheme with user anonymity for network-based e-health care applications. J. Med. Syst. 2016, 40, 256-268. [CrossRef]

52. Wang, X.A.; Ma, J.; Xhafa, F.; Zhang, M.; Luo, X. Cost-effective secure E-health cloud system using identity based cryptographic techniques. Future Gener. Comp. Syst. 2017, 67, 242-254. [CrossRef]

53. Romanou, A. The necessity of the implementation of Privacy by Design in sectors where data protection concerns arise. Comput. Law Secur. Rev. 2018, 34, 99-110. [CrossRef]

54. Wass, S.; Vimarlund, V.; Ros, A. Exploring patients' perceptions of accessing electronic health records: Innovation in healthcare. Health Inform. J. 2019, 25, 203-215. [CrossRef]

55. Glasgow, R.E.; Phillips, S.M.; Sanchez, M.A. Implementation science approaches for integrating eHealth research into practice and Policy. Int. J. Med. Inform. 2014, 83, e1-e11. [CrossRef] [PubMed]

56. Torre-Díez, I.D.L.; Lopez-Coronado, M.; Vaca, C.; Aguado, J.S.; Castro, C. Cost-Utility and cost-effectiveness studies of telemedicine, electronic, and mobile health systems in the literature: A systematic review. Telemed. E-Health 2015, 21, 81-85. [CrossRef]

57. Kleiboer, A.; Smit, J.; Bosmans, J.; Ruwaard, J.; Andersson, G.; Topooco, N.; Berger, T.; Krieger, T.; Botella, C.; Baños, R.; et al. European COMPARative effectiveness research on blended depression treatment versus treatment-as-usual (e-compared): Study protocol for a randomized controlled, non-inferiority trial in eight european countries. Trials 2016, 17, 387. [CrossRef]

58. Provoost, S.; Lau, H.M.; Ruwaard, J.; Riper, H. Embodied conversational agents in clinical psychology: A scoping review: A scoping review. J. Med. Internet Res. 2017, 19, e151. [CrossRef] [PubMed]

59. Devan, H.; Godfrey, H.K.; Perry, M.A.; Hempel, D.; Saipe, B.; Hale, L.; Grainger, R. Current practices of health care providers in recommending online resources for chronic pain self-management. J. Pain Res. 2019, 12, 2457-2472. [CrossRef]

60. Zitkus, V.; Butkiene, R.; Butleris, R.; Maskeliunas, R.; Damasevicius, R.; Wozniak, M. Minimalistic approach to coreference resolution in Lithuanian medical records. Comput. Math. Methods Med. 2019, 2019, 9079840. [CrossRef] [PubMed]

61. Pedersen, S.J.; Cooley, P.D.; Mainsbridge, C. An e-health intervention designed to increase workday energy expenditure by reducing prolonged occupational sitting habits. Work J. Prev. Assess. Rehabil. 2014, 49, 289-295. [CrossRef]

62. Rotondi, A.J.; Eack, S.M.; Hanusa, B.H.; Spring, M.B.; Haas, G.L. Critical design elements of e-health applications for users with severe mental illness: Singular focus, simple architecture, prominent contents, explicit navigation, and inclusive hyperlinks. Schizophr. Bull. 2015, 41, 440-448. [CrossRef]

63. Jackson, B.; Gray, K.; Knowlesd, S.; De Cruz, P. EHealth technologies in inflammatory bowel disease: A systematic review. J. Crohn's Colitis 2016, 10, 1103-1121. [CrossRef]

64. Hollis, C.; Falconer, C.J.; Martin, J.L.; Whittington, C.; Stockton, S.; Glazebrook, C.; Davies, E.B. Digital health interventions for children and young people with mental health problems-A systematic and meta-review. J. Child. Psychol. Psychiatry. 2017, 58, 474-503. [CrossRef] [PubMed]

65. Bricker, J.; Mull, K.; McClure, J.; Watson, N.; Heffner, J. Improving quit rates of web-delivered interventions for smoking cessation: Full-scale randomized trial of WebQuit.org versus Smokefree.gov. Addiction 2018, 113, 914-923. [CrossRef]

66. Jansson, M.M.; Harjumaa, M.; Puhto, A.-P.; Pikkarainen, M. Healthcare professionals' proposed eHealth needs in elective primary fast-track hip and knee arthroplasty journey: A qualitative interview study. J. Clin. Nurs. 2019, 28, 4434-4446. [CrossRef] [PubMed]

67. Mengesha, G.H.; Garfield, M.J. A contextualized IT adoption and use model for telemedicine in Ethiopia. Inf. Technol. Dev. 2019, 25, 184-203. [CrossRef]

68. Pramana, G.; Parmanto, B.; Kendall, P.C.; Skill, J.C. The SmartCAT: An m- health platform for ecological momentary intervention in child anxiety treatment. Telemed. E-Health 2014, 20, 419-427. [CrossRef] [PubMed]

69. World Health Organization. From Innovation to Implementation eHealth in the WHO European Region. Available online: https: //www.euro.who.int/_data/assets/pdf_file/0012/302331/From-Innovation-to-Implementation-eHealth-Report-EU.pdf (accessed on 11 September 2020). 
70. Paige, S.R.; Krieger, J.L.; Stellefson, M.; Alber, J.M. eHealth literacy in chronic disease patients: An item response Theory analysis of the eHealth literacy scale (eHEALS). Patient Educ. Couns. 2017, 100, 320-326. [CrossRef]

71. Holmberg, C.; Berg, C.; Dahlgren, J.; Lissner, L.; Chaplin, J.E. Health literacy in a complex digital media landscape: Pediatric obesity patients' experiences with online weight, food, and health Information. Health Inform. J. 2019, 25, 1343-1357. [CrossRef] [PubMed]

72. Mackert, M.; Champlin, S.E.; Holton, A.; Muñoz, I.I.; Damásio, M.J. Ehealth and health literacy: A research methodology review. J. Comput.-Mediat. Commun. 2014, 19, 516-528. [CrossRef]

73. Diviani, N.; Putte, B.V.D.; Meppelink, C.S.; Diviani, J.C.M.V.W. Exploring the role of health literacy in the evaluation of online health information: Insights from a mixed-methods study. Patient Educ. Couns. 2016, 99, 1017-1025. [CrossRef]

74. Larsen, M.E.; Nicholas, J.; Christensen, H. A systematic assessment of smartphone tools for suicide prevention. PLoS ONE 2016, 11, e0152285. [CrossRef]

75. Malasinghe, L.P.; Ramzan, N.; Dahal, K. Remote patient monitoring: A comprehensive study. J. Ambient Intell. Hum. Comput. 2019, 10, 57-76. [CrossRef]

76. Grist, R.; Porter, J.; Stallard, P. Mental health mobile apps for preadolescents and adolescents: A systematic review. J. Med. Internet Res. 2017, 19, 176. [CrossRef]

77. Lubberding, S.; Uden-Kraan, C.F.V.; Velde, E.A.T.; Cuijpers, P.; Leemans, C.R.; Leeuw, I.M.V. Improving access to supportive cancer care through an eHealth application: A qualitative needs assessment among cancer survivors. J. Clin. Nurs. 2015, 24, 1367-1379. [CrossRef]

78. Schimmer, R.; Orre, C.; Öberg, U.; Danielsson, K.; Hörnsten, A. Digital person-centered self-management support for people with type 2 diabetes: Qualitative study exploring design challenges. JMIR Diabetes 2019, 4, e10702. [CrossRef]

79. Domingues, M.; Alberto, N.; Leitão, C.; Tavares, C.; Lima, E.R.; Radwan, A.; Sucasas, V.; Rodriguez, J.; André, P.; Antunes, P. Insole optical fiber sensor architecture for remote gait analysis-An e-health solution. IEEE Internet Things J. 2019, 6, 207-214. [CrossRef]

80. Moosavi, S.R.; Gia, T.N.; Rahmani, A.-M.; Nigussie, E.; Virtanen, S.; Isoaho, J.; Tenhunen, H. SEA: A secure and efficient authentication and authorization architecture for iot-based healthcare using smart gateways. Procedia Comput. Sci. 2015, 52, 452-459. [CrossRef]

81. Saba, T.; Khan, S.U.; Islam, N.; Abbas, N.; Rehman, A.; Javaid, N.; Anjum, A. Cloud-based decision support system for the detection and classification of malignant cells in breast cancer using breast cytology images. Microsc. Res. Tech. 2019, 82, 775-785. [CrossRef] [PubMed]

82. Liu, X.; Zhou, Y.; Wang, Z. Can the development of a patient's condition be predicted through intelligent inquiry under the e-health business mode? Sequential feature map-based disease risk prediction upon features selected from cognitive diagnosis big data. Int. J. Inf. Manag. 2019, 50, 463-486. [CrossRef]

83. Flodgren, G.; Rachas, A.; Farmer, A.J.; Inzitari, M.; Shepperd, S. Interactive telemedicine: Effects on professional practice and health care outcomes. Cochrane Database Syst. Rev. 2015, 9. [CrossRef]

84. Sultan, N. Making use of cloud computing for healthcare provision: Opportunities and challenges: Opportunities and challenges. Int. J. Inf. Manag. 2014, 34, 177-184. [CrossRef]

85. Brian, R.M.; Ben-Zeev, D. Mobile health (mHealth) for mental health in Asia: Objectives, strategies, and limitations. Asian J. Psychiatry 2014, 10, 96-100. [CrossRef]

86. Yang, H.; Guo, X.; Wu, T. Exploring the influence of the online physician service delivery process on patient satisfaction. Decis. Support. Syst. 2015, 78, 113-121. [CrossRef]

87. Jacob, C.; Sanchez-Vazquez, A.; Ivory, C. Clinicians' role in the adoption of an oncology decision support app in europe and its implications for organizational practices: Qualitative case study: Qualitative case study. JMIR mHealth uHealth 2019, 7, e13555. [CrossRef]

88. Triplett, K.N.; El-Behadli, A.F.; Masood, S.S.; Sullivan, S.; Desai, D.M. Digital medicine program with pediatric solid organ transplant patients: Perceived benefits and challenges. Pediatr. Transplant. 2019, 23, e13555. [CrossRef]

89. Sakakibara, B.M.; Chakrabarti, S.; Krahn, A.; Mackay, M.H.; Sedlak, T.; Singer, J.; Whitehurst, D.G.; Lear, S.A. Delivery of peer support through a self-management mhealth intervention (healing circles) in patients with cardiovascular disease: Protocol for a randomized controlled trial. JMIR Res. Protoc. 2019, 8, e12322. [CrossRef] [PubMed]

90. Brieger, D.; Amerena, J.; Attia, J.R.; Bajorek, B.; Chan, K.H.; Connell, C.; Freedman, B.; Ferguson, C.; Hall, T.; Haqqani, H.M.; et al. National heart foundation of Australia and cardiac society of Australia and New Zealand: Australian clinical guidelines for the diagnosis and management of atrial fibrillation 2018. Med. J. Aust. 2018, 209, 356-362. [CrossRef] [PubMed]

91. Wild, T.S.N.; Fromberger, P.; Jordan, K.; Müller, I.; Müller, J.L. Web-based health services in forensic psychiatry: A review of the use of the internet in the treatment of child sexual abusers and child sexual exploitation material offenders. Front. Psychiatry 2019, 9, 763. [CrossRef] [PubMed]

92. Duruturk, N.; Özköslü, M.A. Effect of tele-rehabilitation on glucose control, exercise capacity, physical fitness, muscle strength and psychosocial status in patients with type 2diabetes: A double blind randomized controlled trial. Prim. Care Diabetes 2019, 13, 542-548. [CrossRef] [PubMed]

93. Van Der Linden, S.; Sitskoorn, M.M.; Rutten, G.-J.M.; Gehring, K. Feasibility of the evidence-based cognitive telerehabilitation program Remind for patients with primary brain tumors. J. Neuro-Oncol. 2018, 37, 523-532. [CrossRef] 
94. Vloothuis, J.D.M.; Mulder, M.; Nijland, R.H.M.; Goedhart, Q.S.; Konijnenbelt, M.; Mulder, H.; Hertogh, C.M.P.M.; Tulder, M.V.; Wegen, E.E.H.V.; Kwakkel, G. Caregiver-mediated exercises with e-health support for early supported discharge after stroke (CARE4STROKE): A randomized controlled trial. PLoS ONE 2019, 14, e0214241. [CrossRef]

95. Kebede, M.; Peters, M.; Heise, T.; Pischke, C. Comparison of three meta-analytic methods using data from digital interventions on type 2 diabetes Diabetes. Diabetes Metab. Syndr. Obes. 2019, 12, 59-73. [CrossRef]

96. Thomas, N.; Bless, J.J.; Alderson-Day, B.; Bell, I.H.; Cella, M.; Craig, T.; Delespaul, P.; Hugdahl, K.; Laloyaux, J.; Larøi, F.; et al. Potential applications of digital technology in assessment, treatment, and self-help for hallucinations. Schizophr. Bull. 2019, 45, S32-S42. [CrossRef]

97. Wang, P.; Ye, F.; Chen, X.; Qian, Y. Datanet: Deep learning based encrypted network traffic classification in SND home gateway. IEEE Access 2018, 6, 55380-55539. [CrossRef]

98. Boussada, R.; Hamdane, B.; Elhdhili, M.E.; Saidane, L.A. Privacy-preserving aware data transmission for IoT-based e-health Comput. Netw. 2019, 162, 106866. [CrossRef]

99. Minoli, D.; Occhiogrosso, B. Blockchain mechanisms for IoT security. Internet Things 2018, 1, 1-13. [CrossRef]

100. Ramu, G.; Reddy, B.E.; Jayanthi, A.; Prasad, L.V.N. Fine-grained access control of EHRs in cloud using CP-ABE with user revocation. Health Technol. 2019, 9, 487-496. [CrossRef]

101. Amin, R.; Islam, S.K.H.; Biswas, G.P.; Khan, M.K.; Li, X. Cryptanalysis and enhancement of anonymity preserving remote user mutual authentication and session key agreement scheme for e-health care systems. J. Med. Syst. 2015, 39, 140. [CrossRef]

102. Waschkau, A.; Wilfling, D.; Steinhaeuser, J. Are big data anaytics helpful in caring for multimorbid patients in general practice?-A scoping review. BMC Fam. Pract. 2019, 20, 37. [CrossRef]

103. Kompara, M.; Kumari, S.; Hölbl, M. Analysis and improvement of a secure key management protocol for e-health application. Comput. Electr. Eng. 2019, 73, 97-113. [CrossRef]

104. Zhou, J.; Cao, Z.; Dong, X.; Lin, X. Security and privacy in cloud-assisted wireless wearable communications: Challenges, solutions and, future directions. IEEE Wirel. Commun. 2015, 22, 136-144. [CrossRef]

105. Wang, J.B.; Cadmus-Bertram, L.A.; Natarajan, L.; White, M.M.; Madanat, H.; Nichols, J.F.; Ayala, G.X.; Pierce, J.P. Wearable sensor/device (fitbit one) and sms text-messaging prompts to increase physical activity in overweight and obese adults: A randomized controlled trial. Telemed. E-Health 2015, 21, 782-792. [CrossRef]

106. Hu, Y.; Bai, G. A systematic literature review of cloud computing in e-health. Health Inform. J. 2014, 3, 11-20. 